\title{
Molecular chemistry approaches for tuning the properties of two-dimensional transition metal dichalcogenides
}

\author{
Simone Bertolazzi, ${ }^{\mathrm{a} *}$ Marco Gobbi, ${ }^{\mathrm{a} \downarrow}$ Y Yda Zhao, ${ }^{\mathrm{a}}$ Claudia Backes ${ }^{\mathrm{b}} \&$ Paolo Samori ${ }^{\mathrm{a}^{*}}$ \\ ${ }^{a}$ Université de Strasbourg, CNRS, ISIS, 8 alleé Gaspard Monge, 67000 Strasbourg, France \\ ${ }^{\mathrm{b}}$ Chair of Applied Physical Chemistry, Ruprecht-Karls University Heidelberg, \\ 69120 Heidelberg, Germany \\ $\$$ Present address: Centro de Física de Materiales (CSIC-UPV/EHU), Paseo Manuel \\ de Lardizabal, 5 -E-20018 Donostia - San Sebastián (Gipzukoa) - Spain \\ *Correspondence should be addressed to: samori@unistr.fr, bertolazzi@unistra.fr
}

\begin{abstract}
Two-dimensional (2D) semiconductors, such as ultrathin layers of transition metal dichalcogenides (TMDs), offer a unique combination of electronic, optical and mechanical properties, with potential to enable a host of new device applications spanning from flexible/wearable (opto)electronics to energy-harvesting and sensing technologies. A critical requirement for developing practical and reliable electronic devices based on semiconducting TMDs is achieving a full control over their charge-carrier polarity and doping. Inconveniently, such a challenging task cannot be accomplished by means of well-established techniques (e.g. ion implantation), which unavoidably damage the $2 \mathrm{D}$ crystals resulting in degraded device performances. Nowadays, a number of alternatives are being investigated, including various (supra)molecular chemistry approaches relying on the combination of 2D semiconductors with electroactive donor/acceptor molecules. As yet, a large variety of molecular systems have been utilized for functionalizing 2D TMDs via both covalent and non-covalent interactions. Such research endeavours enabled not only the tuning of the charge-carrier doping but also the engineering of the optical, electronic, magnetic, thermal and sensing properties of semiconducting TMDs for specific device applications. Here, we will review the most enlightening recent advancements in (supra)molecular chemistry
\end{abstract}


methods for tailoring the properties of atomically-thin TMDs - in the form of substratesupported or solution-dispersed nanosheets - and we will discuss the opportunities and the challenges towards the realization of novel hybrid materials and devices based on 2D semiconductors and molecular systems.

\section{Introduction}

Two-dimensional (2D) materials represent an ever-growing research area that encompasses multiple disciplines and spans from fundamental science to novel device applications. ${ }^{1-3}$ Such a terrific endeavour essentially took off in 2004 with the isolation of graphene from graphite via micromechanical cleavage, also known as scotch tape method. ${ }^{4}$ This simple-yeteffective technique was soon after utilized for the preparation of atomically-thin sheets of various layered van der Waals materials, ${ }^{5}$ including molybdenum disulfide $\left(\mathrm{MoS}_{2}\right)$ and other transition metal dichalcogenides (TMDs). Unlike graphene that is a semimetal and does not possess a bandgap in its pristine form ${ }^{6}-$ a property that is essential for application in digital electronics $^{7}-$, the family of layered-TMD materials offers a broad variety of electronic and optical properties, including semiconducting bandgaps in the visible range of the electromagnetic spectrum. ${ }^{8,9}$ Bulk TMD crystals were investigated back in the $1960 \mathrm{~s},{ }^{10-12}$ and TMD nanosheets with thickness $<100 \AA$ were reported in 1966 by Frindt, who was pioneering the exfoliation of $\mathrm{MoS}_{2}$ by adhesive-tape peeling at that time. ${ }^{13}$ Noticeably, in 1986 - that is almost two decades before the advent of graphene and $2 \mathrm{D}$ crystals $^{4,5}-$ Joensen, Frindt and Morrison ${ }^{14}$ succeeded in preparing single-layer thick sheets of $\mathrm{MoS}_{2}$ via ion-intercalation methods. ${ }^{15,16}$ Nowadays, ultrathin TMDs are among the most popular $2 D$ materials beyond graphene, ${ }^{17,}{ }^{18}$ with monolayer $\mathrm{MoS}_{2}$ being their prototypical and most investigated element. ${ }^{19}$ After the demonstration, in 2011, of dual-gated single-layer $\mathrm{MoS}_{2}$ transistors (ref. 20) with excellent $I_{\mathrm{on}} / I_{\text {off }}$ switching ratio $\left(\sim 10^{8}\right)$ and promising field-effect 
mobility (up to $\sim 60 \mathrm{~cm}^{2} \mathrm{~V}^{-1} \mathrm{~s}^{-1}$ ), ${ }^{21,22}$ tremendous research efforts have been devoted to exploring the use of 2D-TMD semiconductors - e.g. $\mathrm{MoS}_{2}, \mathrm{WS}_{2}, \mathrm{MoSe}_{2}$ and $\mathrm{WSe}_{2}-$ as active layers in electronic switches, such as field-effect transistors (FETs). ${ }^{23-26}$ Thanks to their atomic-scale thicknesses and sizable energy bandgaps $(1-3 \mathrm{eV}),{ }^{27}$ these materials offer ideal electrostatic control in the FET geometry, ${ }^{28,29}$ and for this reason they have been proposed as potential alternatives to silicon for next-generation More-than-Moore technologies. ${ }^{30,31}$ The transition from indirect to direct bandgap that occurs when the thickness of the TMDs is scaled down to a single layer, ${ }^{32-34}$ has also attracted great attention in view of potential applications in optoelectronic devices, ${ }^{26,35-37}$ in particular photodetectors ${ }^{38-40}$ and solar cells. ${ }^{41-}$ ${ }^{44}$ Besides having excellent optical and electronic properties, ultrathin TMDs are also characterized by a high degree of mechanical flexibility, robustness and light weight, ${ }^{45,46}$ which makes them ideal candidates for the fabrication of flexible, foldable and wearable/portable (opto)electronic devices. ${ }^{23,47-49}$

Though impressive advances have been made in this direction, ${ }^{17,}{ }^{18}$ numerous challenges still need to be tackled towards practical and reliable technologies based on 2D semiconductors. ${ }^{1,3}$, ${ }^{23}$ In particular (i) the synthesis of large-area and high-quality films of monolayer TMDs with low densities of defects and impurities, ${ }^{16,50}$ as well as (ii) the development of sound strategies to control the charge-carrier type and the doping level in 2D semiconducting crystals remain major challenges to be faced. ${ }^{1,51-53}$ Whereas a lot of progress has been reported and reviewed on the synthesis of 2D-TMD sheets, ${ }^{16,50,54-56}$ seemingly, relatively less work has been focused on the second critical aspect, which is nevertheless a veritable bottleneck to the incorporation of 2D TMDs in (opto)electronic technologies. ${ }^{1,51-53}$ Achieving a systematic control over charge-carrier doping is essential for minimizing detrimental Schottky barriers at metalsemiconductor interfaces, ${ }^{57}$ as well as for manufacturing $p$ - $n$ junctions and transistors with reproducible electrical characteristics, which are crucial requirements for the production of 
complementary-logic devices and circuits. ${ }^{58}$ Conventional doping techniques, such as ion implantation and dopant diffusion, are extremely challenging to be implemented in combination with 2D TMDs, since damages/defects induced by such processes in ultrathin crystals can have drastic effects on their structural and electrical properties. ${ }^{59-61}$ Various alternatives have been proposed - comprehensively reviewed in refs 51,53 - such as alloying, ${ }^{62}$ transition-metal substitution/incorporation, ${ }^{63-66}$ plasma-assisted doping, ${ }^{67}$ chalcogen substitution via defect engineering, ${ }^{68,69}$ as well as charge transfer via interaction with molecular adlayers. ${ }^{25}$ In particular, the use of molecular systems interfacing 2D semiconducting sheets is emerging as a promising, versatile and viable route for controlling the charge-carrier doping and polarity of ultrathin TMDs. Being essentially all surface, atomically-thin crystals are extremely sensitive to all influences of a surrounding environment, and their properties can thereof be easily modified by external variables. ${ }^{1,70}$ For instance, FETs based on monolayer TMDs sheets were reported to be highly sensitive to a large number of gas molecules, which interact with the surface of the $2 \mathrm{D}$ semiconducting channel modulating its charge-carrier density via electrical dipoles or charge-transfer interactions. ${ }^{71-74}$ Hence, one can take advantage of the exquisite sensitivity of ultrathin TMDs to tune their electronic and optical properties by controlling the physico-chemical variables of the surrounding environment, ${ }^{25,70,75}$ for instance by engineering ad hoc (supra)molecular systems interfacing the $2 \mathrm{D}$ sheets. ${ }^{70,75}$

Indeed, a variety of molecular chemistry approaches (see Fig. 1), based on covalent ${ }^{76-80}$ and non-covalent ${ }^{81-83}$ interactions between molecules and defective/pristine TMDs, have been reported in the last few years. Such studies enabled not only the tuning of the charge-carrier doping $^{81,84-90}$ but also the tailoring of other functional properties critical for (opto)electronic applications, such as for instance charge transport, ${ }^{25,} 64,91-93$ charge injection, ${ }^{94,} 95$ optical 
emission and adsorption, ${ }^{96-103}$ as well as dispersibility in liquid media (inks), ${ }^{104-106}$ the latter being crucial for the processing of large-area films and devices. ${ }^{107,108}$

In this review article, we will describe and discuss the molecular chemistry strategies that have been pursued so far to functionalize 2D TMDs - in particular the so-called MoWSeS materials (i.e. $\mathrm{MoS}_{2}, \mathrm{WS}_{2}, \mathrm{MoSe}_{2}$ and $\left.\mathrm{WSe}_{2}\right)^{2}-$ and to control their charge-carrier doping along with other materials' characteristics. It is worth noting that such strategies have been implemented both on substrate-supported 2D layers (Sections 2-4) and on dispersions of TMD nanosheets in solution (Section 5). More specifically, Section 2 will be focused on noncovalent methods to decorate the top and bottom surfaces of 2D TMDs with electroactive (i.e. donor and acceptor) molecules, which can be eventually deposited in the form of highlyordered molecular layers. ${ }^{83}$ Afterwards, we will show how chemical methods can be employed to control and optimize optical processes in 2D semiconductors, e.g. the photoluminescence emission in direct-bandgap monolayer TMDs (Section 3). Recently, various defect- and phase-engineering approaches have been developed for functionalizing ultrathin TMDs via covalent binding of molecular species onto their basal-plane surface. ${ }^{80,109}$ These techniques will be presented in Section 4. Research endeavours have also been devoted to the development of liquid-phase methods for tailoring the properties of semiconducting nanosheets dispersed in aqueous or organic solutions with a number of molecules and (bio) polymers. ${ }^{110-112}$ The availability of inks of functionalized TMDs is expected to open up novel possibilities for the processing of large-area hybrid materials and devices - e.g. by making use of ink-jet printing methods ${ }^{106}$ - based on combinations of $2 \mathrm{D}$ semiconductors and molecular systems (Section 5). Due to their broader commercial availability and wellestablished exfoliation processes, MoWSeS materials are the most investigated among the TMDs, ${ }^{9}$ and only a few experimental and theoretical works exist on the effects of functional 
molecules on the (opto)electronic properties of other TMD materials (e.g. group-10 $\mathrm{PdSe}_{2}$ and group-5 $\left.\mathrm{NbSe}_{2}\right),{ }^{113,114}$ which have been grouped and reviewed all together in Section 6.

Molecular-design strategies can be used to "bridge" a virtually-infinite number of molecules to $2 \mathrm{D}$ crystals (Section 1.2), providing unique opportunities not only to modulate the properties of ultrathin TMDs - on demand for device applications - but also to introduce new functionalities with the aim of developing novel multifunctional/multiresponsive materials and devices. These opportunities and the associated challenges will be discussed in the last part of this review (Section 7).

\subsection{Materials and properties}

Single sheets of TMDs, commonly indicated with the formula $\mathrm{MX}_{2}$, consist of an atomic layer of transition metals $(\mathrm{M}=\mathrm{Mo}, \mathrm{W}, \mathrm{Ti}, \mathrm{Nb}, \mathrm{Ta}, \mathrm{Re}, \mathrm{Pt}, \mathrm{Pd}$ etc. $)$ sandwiched between two atomic layers of chalcogens $\left(\mathrm{X}=\mathrm{S}\right.$, Se and Te) ${ }^{11,115}$ More than 40 bulk MoX 2 crystals - resulting from different permutations of $\mathrm{M}$ and $\mathrm{X}$ elements - are available in nature, and most of them possess a layered structure. ${ }^{26,115}$ In such layered materials, the atoms within individual sheets are connected via strong covalent bonds (1-10 eV/atom), whereas adjacent sheets interact with each other through weak van-der-Waals forces (10s of meV/atom). ${ }^{1,2,115}$ For this reason, similarly to graphite and other layered materials, ${ }^{116}$ TMDs can be easily exfoliated into singleand few-layer thick nanosheets by making use of various techniques, including micromechanical cleavage via scotch-tape peeling, ${ }^{5,13}$ as well as liquid-phase methods based on intercalation of ions (chemical exfoliation), ${ }^{117-121}$ ultrasonication/shear exfoliation in organic solvents ${ }^{122,123}$ or aqueous surfactant solutions. ${ }^{105}$ Nowadays, graphene is not the only 2D material to be available in the form of high-quality large-area films produced via chemical vapour deposition (CVD). ${ }^{124,125}$ In fact, ultrathin films of MoWSeS materials ${ }^{50,54,126-128}$ and other TMDs (e.g. $\operatorname{ReS}_{2}{ }^{129}$ and $\mathrm{SnSe}_{2}{ }^{130}$ ) can also be grown by CVD methods on technologically-relevant substrates (e.g. oxidized Si wafers), and lateral heterostructure of 
different 2D semiconductors - promising for next-generation (opto)electronic devices - have already been reported in the literature. ${ }^{131,132}$

Nowadays, ultrathin TMDs are intensively investigated for applications in electronic switches, $p$ - $n$ junctions, logic gates and optoelectronic devices. ${ }^{23,27,35}$ In fact, several highquality semiconducting TMDs are currently available with various electronic properties, as well with different charge-transport polarity. For instance, ultrathin $\mathrm{MoS}_{2}$ behaves as a unipolar $n$-type semicductor, whereas $2 \mathrm{D} \mathrm{WSe}_{2}$ commonly displays ambipolarity. ${ }^{27}$

Crystal Structures. TMDs can have multiple crystal structures, depending on the mutual coordination of the $\mathrm{M}$ and $\mathrm{X}$ elements, ${ }^{115,133}$ and transitions between different phases have been predicted ${ }^{134}$ and triggered experimentally via multiple physico-chemical stimuli. ${ }^{135}$ The most common polytypes (see Fig. 2) are the hexagonal - indicated as $1 \mathrm{H}$ for singe layers and $2 \mathrm{H}$ for multilayers - with trigonal prismatic $\left(\mathrm{D}_{3 \mathrm{~h}}\right)$ coordination, and the tetragonal $1 \mathrm{~T}$ with octahedral coordination $\left(\mathrm{O}_{\mathrm{h}}\right) .{ }^{115,133}$ The $1 \mathrm{~T}$ polytype often undergoes a lattice distortion into a $1 \mathrm{~T}^{\prime}$ form, as for instance in the case of $\mathrm{MoTe}_{2} \cdot{ }^{136,137}$ For monolayer MoWSeS, the most stable

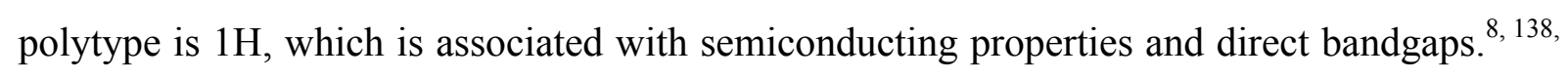
${ }^{139}$ However, metastable $1 \mathrm{~T} / 1 \mathrm{~T}^{\prime}$ polytypes with metallic characteristics can be prepared with various methodologies. ${ }^{135}$ In 1991, Sandoval et al. ${ }^{140}$ demonstrated that monolayer $\mathrm{MoS}_{2}$ sheets produced via lithium-ion intercalation have octahedral phase, and they also proposed a possible $1 \mathrm{~T}^{\prime}$ distorted configuration. Such results were confirmed by a number of subsequent works (e.g. refs 121, 141-143) along with possible strategies for prompting transitions between the metallic $1 \mathrm{~T} / 1 \mathrm{~T}^{\prime}$ and the semiconducting $1 \mathrm{H}$ phases. In 2011 , Eda et al. ${ }^{142}$ reported on photoluminescence emission - typical of $1 \mathrm{H}$ semiconductors - from $\mathrm{MoS}_{2}$ nanosheets produced via lithium-ion intercalation. The key to this achievement was the $1 \mathrm{~T} / 1 \mathrm{~T}^{\prime} \rightarrow 1 \mathrm{H}$ conversion triggered by thermal annealing $\left(T \geq 300^{\circ} \mathrm{C}\right)$ after deposition on a substrate. ${ }^{142}$ This work stimulated the investigation of phase-engineering techniques for 
modulating the properties of ultrathin TMDs, which can be conveniently utilized in combination with molecular chemistry approaches, ${ }^{77,80}$ as discussed in Section 4. The properties and the stability of different polytypes in TMDs "beyond MoWSeS" can vary

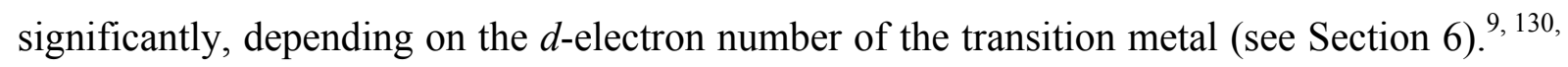
${ }^{131}$ For example, monolayer $\mathrm{PtSe}_{2}$ (group 10) is a 2D semiconductor with indirect bandgap of $\sim 1.2 \mathrm{eV}$ (theory) ${ }^{138,139}$ and 1T stable polytype; ${ }^{144} \mathrm{NbSe}_{2}$ nanosheets (group 4) have stable $1 \mathrm{H} / 2 \mathrm{H}$ metallic phase and were shown to maintain superconducting characteristics down to the monolayer thickness; ${ }^{145}$ single-layer $\mathrm{MoTe}_{2}$ (group 6) displays a $1 \mathrm{~T}^{\prime}$ semimetallic polytype that is almost as stable as its $1 \mathrm{H}$ counterpart, ${ }^{146,147}$ whereas ultrathin $\mathrm{ReS}_{2}$ (group 7) presents a distorted semiconducting $1 \mathrm{~T}^{\prime}$ structure with diamond-shape lines that are responsible for strongly anisotropic transport properties. ${ }^{148,149}$ Here, we will not enter into the details of such a wealth of structures and properties, which have been thoroughly presented elsewhere (e.g. refs 9, 27, 115, 150). However, we point out that the physico-chemical properties of ultrathin TMDs, as well as their interactions with their surroundings, can vary dramatically with the 2D-material structural phase, ${ }^{80}$ and such variability cannot be overlooked when dealing with molecular chemistry methods for the functionalization of ultrathin TMDs.

Defects. Another important aspect to consider is the fact that materials, in general, have defects. Though often regarded as detrimental and undesired due to the fact that they perturb the peculiar material's properties, defects have also been exploited for introducing novel functionalities in (opto)electronic devices, ${ }^{151,152}$ and are being regarded as chemically-active sites suitable for the chemical functionalization of $2 \mathrm{D}$ materials, among which graphene ${ }^{153}$ and ultrathin TMDs. ${ }^{79,88}$ In the case of exfoliated 2D sheets, topological and structural defects can be inherited from the source bulk crystals, and they can also be generated during the exfoliation process. ${ }^{154,155}$ In CVD films, the synthesis/growth leads not only to point defects, 
but also to grain boundaries (GBs), which affect the charge-transport properties in 2D electronic devices. ${ }^{156}{ }^{56}$ Also in this context, $\mathrm{MoS}_{2}$ is the most studied among the TMD crystals. ${ }^{69}$ Its defects and their influences on various physical properties have been extensively investigated, both experimentally ${ }^{157-167}$ and theoretically. ${ }^{165,168-174}$ In comparison, a relatively smaller number of works have focused on other TMDs, and in particular MoWSeS materials. $^{172,175-180}$

Vacancies are a common type of point defects in ultrathin TMD samples and have been found in multiple forms - e.g. single or double, $\mathrm{M}$ or $\mathrm{X}$ vacancies - as recently reviewed by Lin et $a l .{ }^{69}$ In the case of $\mathrm{MoS}_{2}$ monolayers prepared by micromechanical cleavage, aberrationcorrected transmission electron microscopy (TEM) studies were reported in 2013 by Qiu et al., ${ }^{157}$ who showed that sulfur vacancies (SVs) - see Fig. $2 \mathrm{c}$ and $\mathrm{d}-$ are the most abundant type of defects in exfoliated $\mathrm{MoS}_{2}$ nanosheets, and can reach densities of the order of $\sim 10^{13}$ $\mathrm{cm}^{-2}$, corresponding to approximately $10 \mathrm{SV}$ over an area of $10 \times 10 \mathrm{~nm}^{2}$. Two years later, Hong et al., ${ }^{164}$ carried out a systematic TEM investigation and acquired statistical information on the quantities/types of defects in various $\mathrm{MoS}_{2}$ samples. In agreement with the previous work, ${ }^{157}$ they found that single SVs $\left(V_{\mathrm{S} 1}\right)$ can achieve concentrations as high as $1.2 \times 10^{13} \mathrm{~cm}^{-2}$ in both mechanically-exfoliated and CVD-grown $\mathrm{MoS}_{2}$ monolayers. Noticeably, double SVs $\left(V_{\mathrm{S} 2}\right)$ were found to be present mainly in exfoliated nanosheets with densities in the range of $\sim 10^{12} \mathrm{~cm}^{-2} .{ }^{164}$ The landscape of defects/disorder in CVD monolayer $\mathrm{MoS}_{2}$ can be more complex, as shown by Zhou et al. ${ }^{158}$ Besides the most abundant SVs, there are also GBs, dislocations, as well as different types of point defects, such as vacancies of Mo-S complexes and antisite defects. ${ }^{69,158}$

The variety and abundance of defects in $\mathrm{MoS}_{2}$ and other TMDs is being regarded as a major problem to the implementation of practical and reliable (opto)electronic devices based on 2D semiconductors. However, defects can also be exploited to advantage for the chemical 
functionalization of the ultrathin nanosheets. In fact, whereas the basal plane of pristine 2D TMDs lacks of dangling bonds and therefore is rather chemically inert, defect-rich surfaces allow for different chemical reactions to occur, as in the case of SV-containing $\mathrm{MoS}_{2}{ }^{109,}{ }^{181-}$ ${ }^{186}$ It is worth noting that the edges of TMD crystals are also highly chemically reactive and have been widely investigated because of their excellent catalytic properties. ${ }^{187-191}$ Moreover, chalcogen- and metal-rich edges are being regarded as suitable targets for the chemical functionalization of liquid-phase exfoliated nanosheets (see Section 5), ${ }^{192-196}$ enabling the production of functional TMD inks for (opto)electronic ${ }^{197}$ as well as biomedical applications. ${ }^{198}$

In the last few years, several defect-engineering strategies have been developed for tailoring the physico-chemical properties of 2D TMDs, ${ }^{69}$ opening new routes for the functionalization of TMDs via molecular chemistry methods (Section 4.1).

\section{2 "Bridging" ultrathin TMDs with molecular systems}

Numerous advantages arise from the functionalization of ultrathin TMDs with molecular systems. Besides offering unique opportunities for tailoring the electrical, optical, thermal and sensing properties of TMDs, such a combination makes it also possible to develop novel multifunctional/multiresponsive hybrid materials and devices suitable for advanced logic, memory and sensing applications. ${ }^{199}$ Indeed, functionalization methods aiming at "bridging" these two worlds have been intensively investigated over the past few years. ${ }^{76,111,198,200}$ In particular, molecular chemistry offers a variety of techniques to achieve this objective, as illustrated in Fig. 1. The simplest technique is based on the physisorption of electroactive molecules on substrate-supported nanosheets, which can be achieved via solution-processing (e.g. refs 87,201$)$ or vapour-phase (e.g. ref. 202) deposition. A more elaborated approach consists in the use of the principles of supramolecular chemistry to control the ordering of molecules via self-assembly. ${ }^{83,203}$ Highly-ordered crystalline assemblies of physisorbed 
molecules can be grown on 2D materials by drop casting or spin coating, ${ }^{93,203}$ resulting in spatially uniform periodic interactions between the $2 \mathrm{D}$ sheets and the molecular adlayers. ${ }^{93}$ In addition, the packing and orientation of the molecules can be engineered ad hoc in order to modulate the magnitude/direction of surface dipoles, as well as the charge transfer between the molecular layers and the $2 \mathrm{D}$ crystal. ${ }^{93}$ This represents an intriguing opportunity for tuning the charge-carrier doping in ultrathin TMDs, eventually by bringing into play cooperativity or collective processes. ${ }^{204}$ A slightly different technique involves the preparation of selfassembled monolayers (SAMs) via molecular chemisorption on suitable substrates, followed by the deposition/transfer of the $2 \mathrm{D}$ sheets (e.g. refs 84,92$)$. More in general, the TMDs' electronic and optical properties can be tailored by controlling the substrate surface chemistry, which can be accomplished with multiple approaches, including the use of polymer coating layers. $^{205,206}$

In all the cases discussed above, the functionalization is achieved through non-covalent interactions between the molecules and the 2D sheets. However, it is also possible to "bridge" ultrathin TMDs and molecules via strong and stable covalent bonds, e.g. by exploiting defect and phase-engineering approaches ${ }^{69,207}$ to activate and harness the chemical reactivity of the TMDs' surface. For instance, ultrathin layers of $\mathrm{MoS}_{2}$ containing SVs can be "decorated" with molecules bearing nucleophilic functional groups - especially thiols ${ }^{88,109}$ - that can coordinate/bind to the exposed molybdenum atoms at SV sites. ${ }^{181,183,184}$ Whereas the semiconducting $1 \mathrm{H} / 2 \mathrm{H}$ phase of $\mathrm{MoS}_{2}$ cannot be easily functionalized in the absence of defects, ${ }^{110}$ its electron-rich $1 \mathrm{~T} / 1 \mathrm{~T}^{\prime}$ counterpart allows for numerous functionalization routes, ${ }^{111}$ since the occurrence of bond-forming reactions is facilitated by electron transfer between the metallic sheets and the reactant species (e.g. electrophiles such as organohalides). ${ }^{80}$ Hence, one can exploit phase-conversion processes in TMDs ${ }^{135}$ (see Section 1.1) to obtain metallic polytypes suitable for chemical functionalization, and eventually 
convert them back to their original phase, as demonstrated by Voiry et al. ${ }^{80}$ Noticeably, all these approaches can be conducted directly in solution - e.g. in the case of liquid-phase exfoliated TMDs (Section 5) - or on substrate-supported sheets, as outlined in detail below.

\section{Tuning the properties of TMDs via non-covalent interactions}

In conventional semiconductor technology, precise control over the optoelectronic properties of materials can be achieved by substitutional doping, which consists in replacing host atoms of the crystal with dopant impurities. ${ }^{58,151}$ In ultrathin TMDs, doping can be accomplished by decorating their surface with electroactive (i.e. donor or acceptor) molecules, which can be used to controllably tune the charge-carrier density through diverse electromagnetic interactions (e.g. electrostatic and van der Waals). Indeed, the ultra-high surface-to-volume ratio in 2D TMDs makes their optoelectronic properties extremely sensitive to any variation in their surface environment. While substitutional doping has also been successfully applied to TMDs, ${ }^{63,65,79,208}$ the non-covalent molecular approach (i.e. physisorption) has the advantage of leaving the crystal structure of TMDs unaltered, preserving their superior optoelectronic properties.

Two major mechanisms lead to the molecular doping of TMDs, which are (i) charge transfer and (ii) dipolar interactions. Charge transfer occurs when the electrochemical redox potential of the molecules and the Fermi level of 2D materials comply with certain conditions. When the redox potential of a given molecular dopant lies below the TMD Fermi level, electron transfer can occur from the TMD sheet to the molecule, resulting in $p$-type doping. ${ }^{209}$ Vice versa, when the redox potential of molecules lies above the TMD Fermi level, electrons are transferred from the molecules to the TMD, inducing $n$-type doping. ${ }^{85}$ Fig. 3 compares the reduction potentials of various molecular dopants with the band edges - namely the electron affinity (conduction band minimum, $\mathrm{CBM}$ ) and the ionization potential (valence band maximum, VBM) - of group-6 monolayer TMDs. ${ }^{210}$ Although the exact Fermi level position 
of TMDs depends on several factors (e.g. impurities, substrate, crystal defects, etc.), the figure provides a guideline for the choice of suitable molecules to realize doping in monolayer TMD sheets based on possible charge-transfer processes involving the CBM/VBM levels of TMDs. It should be noticed that the band edges are characterized by a significant uncertainty; for instance, the electron affinity of $1 \mathrm{~L} \mathrm{MoS}_{2}$ has been reported to vary from $\sim 3.9 \mathrm{eV}$ (ref. 211) to $\sim 4.7 \mathrm{eV}$ (ref. 212).

Dipolar interactions can also be exploited for tuning the charge-carrier doping in ultrathin TMDs. Molecules with permanent dipole moments generate electric fields on the 2D-sheet surface, modulating the (local) charge carrier density similarly to nanoscopic electric gates. If the dipole moments of several molecules lying on the TMD surface possess the same spatial orientation, single-molecule effects can sum up leading to a macroscopic modification of the TMD energetics. In particular, a shift in the Fermi level of semiconducting TMDs was observed as a consequence of the interaction with well-aligned molecular dipoles, ${ }^{84}$ resulting in $p$ - or $n$ - type doping depending on the dipole direction. ${ }^{86}$ In most cases, both mechanisms contribute to the doping effect, and it is often difficult to disentangle the two contributions. ${ }^{92}$ In this section, we will summarize the doping of TMDs through the decoration of their top and bottom surfaces with electroactive molecular systems. As an overview, Table 1 provides a summary of the molecules that have been used to decorate ultrathin TMDs, highlighting their impact on the optoelectronic and vibrational properties, as well as the mechanisms responsible for the measured effects. 
Table 1. Effects of molecular dopants on the electronic (doping), vibrational ( $\mathrm{A}_{1 \mathrm{~g}}$, and $\mathrm{E}_{2 \mathrm{~g}}^{1}$ Raman modes) and optical (PL) properties of substratesupported ultrathin TMDs. Notes: CT, charge transfer; DI, dipolar interactions; PI-CT, photo-induced charge transfer; RS, red shift; BS, blue shift; FWHM, full width at half maximum. (*) TMD coated with a hybrid PMMA/F - TCNQ layer.

\begin{tabular}{|c|c|c|c|c|c|c|c|c|c|c|}
\hline Ref. & Molecules & TMD & $\begin{array}{c}\text { Doping } \\
\text { mechanisms }\end{array}$ & $\begin{array}{l}\text { Doping } \\
\text { type }\end{array}$ & $\begin{array}{c}\text { Doping } \\
\text { density } \\
\Delta Q / \mathrm{e}\left[\mathrm{cm}^{-2}\right]\end{array}$ & $\begin{array}{l}\text { Effect } \\
\text { on PL }\end{array}$ & $\begin{array}{c}\text { Effect on } \\
\text { Raman } \\
\text { modes }\end{array}$ & $\begin{array}{c}\text { Functional group } \\
\text { interacting with } \\
\text { TMDs }\end{array}$ & Stability & $\begin{array}{c}\text { Deposition } \\
\text { method }\end{array}$ \\
\hline & Decoration of the top surface & & & & & & & & & \\
\hline 85 & benzyl viologen (BV) & $\mathrm{MoS}_{2}$ & $\mathrm{CT}$ & $n$ & $\sim 10^{13}$ & & $\begin{array}{l}\mathrm{A}_{1 \mathrm{~g}} \mathrm{RS} \\
\mathrm{FWHM} \uparrow\end{array}$ & & 9 days & $\begin{array}{l}\text { drop cast or } \\
\text { soaking }\end{array}$ \\
\hline 209 & nicotinamide adenine dinucleotide (NADH) & $\mathrm{MoS}_{2}$ & $\mathrm{CT}$ & $n$ & & $\mathrm{RS}, \mathrm{I} \downarrow$ & & $-\mathrm{NH}_{2}$ & & drop cast \\
\hline 88 & mercaptoethylamine (MEA) & $\mathrm{MoS}_{2}$ & $\mathrm{CT}$ & $n$ & $3.7 \times 10^{12}$ & $\mathrm{RS}, \mathrm{I} \downarrow$ & & $-\mathrm{NH}_{2}$ & 1 week & soaking \\
\hline 94 & polyethyleneimine (PEI) & $\mathrm{MoS}_{2}$ & $\mathrm{CT}$ & $n$ & & & & $-\mathrm{NH}_{2}$ & unstable & soaking \\
\hline $\begin{array}{l}101 \\
101 \\
213\end{array}$ & triphenylphosphine $\left(\mathrm{PPh}_{3}\right)$ & $\begin{array}{l}\mathrm{MoS}_{2} \\
\mathrm{ReSe}_{2} \\
\mathrm{WSe}_{2}\end{array}$ & $\begin{array}{l}\text { CT } \\
\text { CT } \\
\text { CT }\end{array}$ & $\begin{array}{l}n \\
n \\
n\end{array}$ & $\begin{array}{l}1.6 \times 10^{11} \\
1.1 \times 10^{11} \\
7.8 \times 10^{11}\end{array}$ & & $\begin{array}{c}A_{1 g}, E_{2 g}^{1} R S, \\
A_{1 g} F W H M \\
\uparrow \\
A_{g}, E_{g} R S, \\
A_{g} \text { FWHM } \uparrow \\
A_{1 g}, E_{2 g}^{1} B S\end{array}$ & & $\begin{array}{l}14 \text { days } \\
14 \text { days } \\
8 \text { days }\end{array}$ & $\begin{array}{l}\text { spin-coating } \\
\text { spin-coating } \\
\text { spin-coating }\end{array}$ \\
\hline 214 & hydrazine & $\mathrm{WSe}_{2}$ & $\mathrm{CT}$ & $n$ & & & $A_{1 g}, E_{2 g}^{1} R S$ & $-\mathrm{NH}_{2}$ & & soaking \\
\hline 88 & 1H,1H,2H,2H-perfluorodecanethiol (FDT) & $\mathrm{MoS}_{2}$ & $\mathrm{CT}$ & & $1.8 \times 10^{11}$ & $\mathrm{BS}, \mathrm{I} \uparrow$ & & $-\mathrm{CF}_{3}$ & & soaking \\
\hline 209 & $\begin{array}{l}\text { tetracyanoquinodimethane } \\
\text { (TCNQ) }\end{array}$ & $\mathrm{MoS}_{2}$ & $\mathrm{CT}$ & $p$ & & $\mathrm{BS}, \mathrm{I} \uparrow$ & & & & drop cast \\
\hline
\end{tabular}




\begin{tabular}{|c|c|c|c|c|c|c|c|c|c|c|}
\hline $\begin{array}{l}209 \\
215 \\
216\end{array}$ & $\begin{array}{l}\text { tetrafluoro-7,7,8,8-tetracyanoquinodimethane } \\
\left(\mathrm{F}_{4}-\mathrm{TCNQ}\right)\end{array}$ & $\begin{array}{l}\mathrm{MoS}_{2} \\
\mathrm{WSe}_{2} \\
\mathrm{WS}_{2}\end{array}$ & $\begin{array}{l}\mathrm{CT} \\
\mathrm{CT} \\
\mathrm{CT}\end{array}$ & $\begin{array}{l}p \\
p \\
p\end{array}$ & $\begin{array}{l}5.8 \times 10^{13} \\
7.4 \times 10^{13}\end{array}$ & $\mathrm{BS}, \mathrm{I} \uparrow$ & $\mathrm{A}_{1 \mathrm{~g}}, \mathrm{BS}$ & $\begin{array}{l}-F \\
-F \\
-F\end{array}$ & 2 weeks & $\begin{array}{l}\text { drop cast } \\
\quad(*) \\
\text { drop cast }\end{array}$ \\
\hline $\begin{array}{l}99 \\
99 \\
99\end{array}$ & magnesium phtalocyanine (MgPc) & $\begin{array}{c}\mathrm{MoS}_{2} \\
\mathrm{MoSe}_{2} \\
\mathrm{WSe}_{2}\end{array}$ & $\mathrm{PI}-\mathrm{CT}$ & $p$ & & $\mathrm{I} \downarrow$ & & & & $\begin{array}{l}\text { soaking soaking } \\
\text { soaking }\end{array}$ \\
\hline $\begin{array}{l}99 \\
99 \\
99\end{array}$ & nickel phtalocyanine (NiPc) & $\begin{array}{c}\mathrm{MoS}_{2} \\
\mathrm{MoSe}_{2} \\
\mathrm{WSe}_{2}\end{array}$ & $\begin{array}{l}\text { PI-CT } \\
\text { PI-CT }\end{array}$ & $\begin{array}{l}p \\
p\end{array}$ & & $\begin{array}{l}\mathrm{I} \downarrow \\
\mathrm{I} \downarrow\end{array}$ & & & & $\begin{array}{l}\text { soaking soaking } \\
\text { soaking }\end{array}$ \\
\hline 217 & titanyl phthalocyanine (TiOPc) & $\mathrm{MoS}_{2}$ & $\mathrm{CT}$ & $p$ & & $\mathrm{I} \uparrow$ & & & & evaporation \\
\hline 39,86 & octadecyltrichlorosilane (ODTS) & $\begin{array}{l}\mathrm{WSe}_{2} \\
\mathrm{MoS}_{2}\end{array}$ & $\begin{array}{l}\text { DI } \\
\text { DI }\end{array}$ & $\begin{array}{l}p \\
p\end{array}$ & $\begin{array}{l}1.8 \times 10^{11} \\
1.4 \times 10^{11}\end{array}$ & $\begin{array}{l}\mathrm{I} \downarrow \\
\mathrm{I} \uparrow\end{array}$ & $\begin{array}{l}A_{1 g}, E_{2 g}^{1} B S \\
A_{1 g}, E_{2 g}^{1} B S\end{array}$ & $\begin{array}{l}-\mathrm{CH}_{3} \\
-\mathrm{CH}_{3}\end{array}$ & 100 hours & soaking soaking \\
\hline $\begin{array}{l}39 \\
39\end{array}$ & 3-(trimethoxysilyl)-1-propanamine (APTES) & $\begin{array}{l}\mathrm{WSe}_{2} \\
\mathrm{MoS}_{2}\end{array}$ & $\begin{array}{l}\mathrm{DI} / \mathrm{CT} \\
\mathrm{DI} / \mathrm{CT}\end{array}$ & $\begin{array}{l}n \\
n\end{array}$ & $\begin{array}{c}1.1 \times 10^{11} \\
1 \times 10^{11}\end{array}$ & $\begin{array}{l}\mathrm{I} \uparrow \\
\mathrm{I} \downarrow\end{array}$ & $\begin{array}{l}A_{1 g}, E_{2 g}^{1} R S \\
A_{1 g}, E_{2 g}^{1} R S\end{array}$ & $\begin{array}{l}-\mathrm{NH}_{2} \\
-\mathrm{NH}_{2}\end{array}$ & & soaking soaking \\
\hline 81 & oleylamine & $\mathrm{MoS}_{2}$ & $\mathrm{DI} / \mathrm{CT}$ & $n$ & $1.8 \times 10^{13}$ & $\mathrm{RS}$ & $\mathrm{A}_{1 \mathrm{~g}} \mathrm{RS}$ & $-\mathrm{NH}_{2}$ & & soaking \\
\hline 102 & methyl salicylate & $\mathrm{MoS}_{2}$ & & $p$ & & $\mathrm{I} \uparrow$ & $\mathrm{A}_{1 \mathrm{~g}} \mathrm{BS}$ & & & spin-coating \\
\hline 218 & perylenediimides & $\mathrm{MoS}_{2}$ & $\mathrm{CT}$ & $n$ & & & & & & drop cast \\
\hline 218 & tetraphenyl porphyrins & $\mathrm{MoS}_{2}$ & $\mathrm{CT}$ & $n$ & & & & & & drop cast \\
\hline 102 & benzoic acid & $\mathrm{MoS}_{2}$ & & $p$ & & $\mathrm{I} \uparrow$ & $\mathrm{A}_{1 \mathrm{~g}} \mathrm{BS}$ & $-\mathrm{COOH}$ & & spin-coating \\
\hline 102 & phenol & $\mathrm{MoS}_{2}$ & & $p$ & & $\mathrm{I} \uparrow$ & $\mathrm{A}_{1 \mathrm{~g}} \mathrm{BS}$ & $-\mathrm{OH}$ & & spin-coating \\
\hline 102 & salicylic acid & $\mathrm{MoS}_{2}$ & & $p$ & $9 \times 10^{13}$ & $\mathrm{I} \uparrow$ & $\mathrm{A}_{1 \mathrm{~g}} \mathrm{BS}$ & $-\mathrm{COOH}$ & & spin-coating \\
\hline 102 & nitrobenzene & $\mathrm{MoS}_{2}$ & & $p$ & & $\mathrm{I} \uparrow$ & $\mathrm{A}_{1 \mathrm{~g}} \mathrm{BS}$ & $-\mathrm{NO}_{2}$ & & spin-coating \\
\hline
\end{tabular}




\begin{tabular}{|c|c|c|c|c|c|c|c|c|c|}
\hline 102 & aniline & $\mathrm{MoS}_{2}$ & & $p$ & & $\mathrm{I} \uparrow$ & $\mathrm{A}_{1 \mathrm{~g}} \mathrm{BS}$ & $-\mathrm{NH}_{2}$ & spin-coating \\
\hline 102 & melamine & $\mathrm{MoS}_{2}$ & & $p$ & & $\mathrm{I} \uparrow$ & $\mathrm{A}_{1 \mathrm{~g}} \mathrm{BS}$ & $-\mathrm{NH}_{2}$ & spin-coating \\
\hline 219 & $\begin{array}{l}\text { fluorinated fullerene } \\
\left(\mathrm{C}_{60} \mathrm{~F}_{48}\right)\end{array}$ & $\mathrm{WSe}_{2}$ & $\mathrm{CT}$ & $p$ & $1 \times 10^{12}$ & \multirow{8}{*}{ none } & \multirow{3}{*}{ none } & $-F$ & evaporation \\
\hline 84 & octyltrichlorosilane (OTS) & $\mathrm{MoS}_{2}$ & DI & none & none & & & $-\mathrm{CH}_{3}$ & SAM \\
\hline 92 & octadecyltrichlorosilane (ODTS) & $\mathrm{MoS}_{2}$ & DI & $p$ & $5 \times 10^{11}$ & & & $-\mathrm{CH}_{3}$ & SAM \\
\hline 220 & DNA & $\begin{array}{l}\mathrm{MoS}_{2} \\
\mathrm{WSe}_{2}\end{array}$ & $\begin{array}{l}\text { DI } \\
\text { DI }\end{array}$ & $\begin{array}{l}n \\
n\end{array}$ & $\begin{array}{l}6 \times 10^{10} \\
7 \times 10^{9}\end{array}$ & & $\begin{array}{l}A_{1 g}, E^{1}{ }_{2 g} R S \\
A_{1 g}, E_{2 g}^{1} R S\end{array}$ & phosphate & drop cast \\
\hline $\begin{array}{l}220 \\
221\end{array}$ & metal DNA & $\begin{array}{l}\mathrm{MoS}_{2} \\
\mathrm{WSe}_{2}\end{array}$ & $\begin{array}{l}\text { DI } \\
\text { DI }\end{array}$ & $\begin{array}{l}p / n \\
p / n\end{array}$ & $1-5 \times 10^{10}$ & & $\begin{array}{l}A_{1 g}, E_{2 g}^{1} B S \\
A_{1 g}, E_{2 g}^{1} B S\end{array}$ & \multirow[t]{4}{*}{$\begin{array}{l}\mathrm{Zn}, \mathrm{Ni}, \mathrm{Co}, \mathrm{Cu}, \mathrm{Eu} \\
\quad \mathrm{Gd}, \mathrm{Tb}, \mathrm{Er}\end{array}$} & drop cast \\
\hline 87 & $\begin{array}{l}\text { tris(4-bromophenyl)ammoniumyl } \\
\text { hexachloroantimonate (Magic Blue) }\end{array}$ & $\mathrm{MoS}_{2}$ & $\mathrm{CT}$ & $p$ & $8 \times 10^{12}$ & & $A_{1 g}, E_{2 g}^{1} B S$ & & dip coating \\
\hline 87 & $\begin{array}{l}\text { 2-ferrocenyl N,N'-dimethylbenzimidazoline } \\
\left((2-\mathrm{Fc}-\mathrm{DMBI})_{2}\right)\end{array}$ & $\mathrm{MoS}_{2}$ & $\mathrm{CT}$ & $n$ & $6.3 \times 10^{12}$ & & $A_{1 g}, E_{2 g}^{1} R S$ & & dip coating \\
\hline 87 & $\begin{array}{l}\text { 2-ferrocenyl-2,3-dihydro-1H- } \\
\text { benzimidazoline (2-Fc-DMBI-H) }\end{array}$ & $\mathrm{MoS}_{2}$ & CT & $n$ & $5.2 \times 10^{12}$ & & $A_{1 g}, E_{2 g}^{1} R S$ & & dip coating \\
\hline \multicolumn{10}{|c|}{ Decoration of the bottom surface } \\
\hline $\begin{array}{l}84 \\
92\end{array}$ & 3-(trimethoxysilyl)-1-propanamine (APTES) & $\begin{array}{l}\mathrm{MoS}_{2} \\
\mathrm{MoS}_{2}\end{array}$ & $\begin{array}{c}\mathrm{DI} \\
\mathrm{DI} / \mathrm{CT}\end{array}$ & $\begin{array}{l}n \\
n\end{array}$ & $1 \times 10^{11}$ & $\mathrm{I} \uparrow$ & $\begin{array}{l}\mathrm{A}_{1 \mathrm{~g}} \mathrm{RS} \\
\mathrm{FWHM} \uparrow\end{array}$ & $\begin{array}{l}-\mathrm{NH}_{2} \\
-\mathrm{NH}_{2}\end{array}$ & $\begin{array}{l}\text { SAM } \\
\text { SAM }\end{array}$ \\
\hline 84 & trichloro- $(1 \mathrm{H}, 1 \mathrm{H}, 2 \mathrm{H}, 2 \mathrm{H}-$ & $\mathrm{MoS}_{2}$ & DI & $p$ & & & $\mathrm{~A}_{1 \mathrm{~g}} \mathrm{BS}$ & $-\mathrm{CF}_{3}$ & SAM \\
\hline 92 & perfluorooctyl)silane (FOTS) & $\mathrm{MoS}_{2}$ & $\mathrm{DI} / \mathrm{CT}$ & $n$ & $8 \times 10^{11}$ & & FWHM $\downarrow$ & $-\mathrm{CF}_{3}$ & SAM \\
\hline 92 & (3-mercaptopropyl)trimethoxysilane (MPS) & $\mathrm{MoS}_{2}$ & $\mathrm{DI} / \mathrm{CT}$ & $n$ & $1 \times 10^{11}$ & $\mathrm{I} \uparrow$ & & $-\mathrm{SH}$ & SAM \\
\hline 222 & $\begin{array}{l}\text { (E)-6-(4-(phenyldiazenyl)phenoxy)hexane-1- } \\
\left.\text { thiol (HS- } \mathrm{C}_{6} \mathrm{AZO}\right)\end{array}$ & $\mathrm{MoS}_{2}$ & $\mathrm{DI} / \mathrm{CT}$ & $n$ & & $\mathrm{I} \downarrow$ & & -phenyl & SAM \\
\hline 222 & $\begin{array}{l}\text { (E)-6- (4-((4-chlorophenyl)diazenyl)- } \\
\left.\text { phenoxy)hexane-1-thiol (HS- } \mathrm{C}_{6} \mathrm{AZO}-\mathrm{Cl}\right)\end{array}$ & $\mathrm{MoS}_{2}$ & $\mathrm{DI} / \mathrm{CT}$ & $p$ & & $\mathrm{I} \uparrow$ & & $-\mathrm{Cl}$ & SAM \\
\hline
\end{tabular}




\subsection{Physisorption of electroactive molecules}

Crystal defects and impurities introduced with conventional doping techniques can significantly affect the electrical characteristics of FETs based on monolayer TMDs, including the field-effect mobility, the threshold voltage and the subthreshold swing. ${ }^{109,223,224}$ Moreover, they can degrade the performances of optoelectronic devices in terms of photoresponsivity and light emission. ${ }^{175,225}$ The physisorption of electroactive molecules on the surface of ultrathin TMD sheets represents an alternative non-destructive method for tuning doping via charge transfer, as discussed previously. One of the most studied molecules is 2,3,5,6-tetrafluoro-7,7,8,8-tetracyanoquinodimethane ( $\mathrm{F}_{4}$-TCNQ), which is a common electron acceptor (p-dopant). ${ }^{209}$ The redox potential of $\mathrm{F}_{4}$-TCNQ lies below the CBM of monolayer $\mathrm{MoS}_{2}$ (see Fig. 3) so that electron transfer can occur from the conduction band of heavily $n$-doped $\mathrm{MoS}_{2}$ - where the Fermi level lies close to the CBM - to the unoccupied energy states of the $\mathrm{F}_{4}$-TCNQ molecules, resulting in electron depletion. The $p$-doping effect is evident from the evolution of the photoluminescence (PL) spectra upon molecular physisorption (Fig. 4a). The A exciton, which is associated with optical transitions at the K point of the Brillouin zone, consists of both negatively-charged excitons (i.e. trions, $E\left(\mathrm{X}^{-}\right)$ $\approx 1.84 \mathrm{eV})$ and neutral excitons $(E(\mathrm{X}) \approx 1.88 \mathrm{eV})$, so that the trion spectral weight depends on the amount of doping in the $2 \mathrm{D}$ semiconducting sheet. ${ }^{226}$ For the as-exfoliated $\mathrm{MoS}_{2}$, the trion intensity was found to be higher than that of the neutral exciton (see fitting in Fig. 4c) due to the $n$-type doping typically occurring in natural $\mathrm{MoS}_{2}$ crystals. ${ }^{19}$ On the other hand, in the $\mathrm{F}_{4^{-}}$ TCNQ-doped $\mathrm{MoS}_{2}$ the contribution of the neutral exciton becomes dominant due to electron depletion, the A peak blue shifts and its intensity increases dramatically.

Different from $\mathrm{F}_{4}$-TCNQ, nicotinamide adenine dinucleotide (NADH) has a redox potential higher than the CBM of monolayer $\mathrm{MoS}_{2}$ (see Fig. 3) and therefore can be used for $n$-type doping. After physisorption of NADH molecules, the PL spectra of monolayer $\mathrm{MoS}_{2}$ (Fig. 4b) 
are characterized by a higher trion spectral weight. Moreover, the A peak red-shifts and its intensity decreases, due to electron accumulation in the 2D material. Hence, through the molecular physisorption approach both the charge-carrier doping and the PL emission spectra can be effectively modulated.

Similar results were obtained by Peimyoo et al. ${ }^{216}$ by making use of monolayer (1L) $\mathrm{WS}_{2}$ sheets in combination with $\mathrm{F}_{4}$-TCNQ molecules. The authors investigated the effects of molecular physisorption on the electrical properties of back gated 1L-WS 2 FETs (Fig. 4d). With increasing $\mathrm{F}_{4}$-TCNQ concentration, the threshold voltage was found to shift towards more positive gate-voltage values in line with the occurrence of $p$-type doping.

Besides PL and charge-transport measurements, Raman spectroscopy is also a versatile tool for studying doping effects in TMDs. ${ }^{227}$ The two main Raman-active phonon modes of $2 \mathrm{H}-$ TMD crystals are $A_{1 \mathrm{~g}}$ and $E_{2 \mathrm{~g}}^{1}$, which correspond to out-of-plane vibrations of chalcogen atoms and in-plane vibrations of chalcogen and transition metal atoms, respectively. ${ }^{228}$ Density functional theory (DFT) calculations and symmetry arguments predict that for $\mathrm{MoS}_{2}$, the $A_{1 \mathrm{~g}}$ phonon mode is more sensitive to the electron doping than the $E_{2 \mathrm{~g}}^{1}$ due to a stronger electron-phonon coupling in the out-of-plane $A_{1 \mathrm{~g}}$ vibration. ${ }^{227}$ Raman spectra of few-layer $\mathrm{MoS}_{2}$ sheets were measured after "decoration" with $n$-type and $p$-type dopant molecules, as illustrated in Fig. $5 .{ }^{87}$ For the $n$-type dopant $(2-\mathrm{Fc}-\mathrm{DMBI})_{2}-$ i.e. 2-ferrocenyl N,N'dimethylbenzimidazoline radical, inset of Fig. $5 \mathrm{~b}-$ the $A_{1 \mathrm{~g}}$ peak of $\mathrm{MoS}_{2}$ red-shifts by $\sim 1$ $\mathrm{cm}^{-1}$ and the peak broadens $\left(\triangle \mathrm{FWHM} \approx 1.4 \mathrm{~cm}^{-1}\right)$, whereas the $E_{2 \mathrm{~g}}^{1}$ mode remains almost unchanged $\left(\Delta v<0.2 \mathrm{~cm}^{-1}\right.$, Fig. 5b). In contrast, for $p$-type dopants such as tris(4bromophenyl)ammoniumyl hexachloroantimonate ("Magic Blue", Fig. 5d) both $A_{1 \mathrm{~g}}$ and $E_{2 \mathrm{~g}}^{1}$ peak blue-shift $\left(\Delta v \approx 0.5 \mathrm{~cm}^{-1}\right)$ and the peak sharpens $\left(\Delta \mathrm{FWHM} \approx-0.4 \mathrm{~cm}^{-1}\right)$. The effects of molecular physisorption doping on the Raman spectra of $\mathrm{MoS}_{2}$ nanosheets are similar to those induced by electrostatic gating in FET structures. ${ }^{227}$ With the increasing gate voltage (electron 
doping), the $A_{1 \mathrm{~g}}$ peak red-shifts and the peak broadens. This demonstrates that molecular physisorption influence phonon vibrations by modifying electron-phonon interactions. Furthermore, the shift of the $A_{1 \mathrm{~g}}$ Raman peak is proportional to the doping concentration, ${ }^{227}$ which is quite useful to probe the doping level in TMDs.

Numerous molecules have been exploited to tune the properties of TMDs. ${ }^{25,36}$ Among these, we discuss a class of metallophthalocyanine molecules - indicated as MPc, where M is the metal centre (e.g. NaPc, MgPc, TiPc, FePc, NiPc, CuPc, PtPc, etc.) - which are currently attracting attention for doping TMDs due to their metal-centre dependent redox potential. ${ }^{99} \mathrm{~A}$ schematic illustration of a MPc molecule physisorbed on a TMD sheet is provided in Fig. 6 a. The planar structure of $\pi$-conjugated rings results in a flat-lying MPc/TMD configuration. Based on the positions of the band edges of TMDs with respect to the redox potential of MPcs, it is possible to predict the direction of the charge transfer between the TMDs and the MPc molecules. ${ }^{99}$ For example, the redox potential of NiPc $(\mathrm{MgPc})$ is located below (above) the CBM of $\mathrm{MoSe}_{2}$ (Fig. 3). Upon light irradiation, photo-excited electrons in the conduction band of $\mathrm{MoSe}_{2}$ can transfer to NiPc molecules, which provide a non-radiative recombination pathway that severely degrades the PL quantum yield of monolayer $\mathrm{MoSe}_{2}$ (Fig. 6b). In contrast, no significant changes can be observed in the PL spectra of MgPc-treated $\mathrm{MoSe}_{2}$ (Fig. 6c). ${ }^{99}$ Physisorbed MPc molecules can be effectively used for tuning the charge-carrier density in ultrathin TMD sheets. For instance, NaPc induces $n$-type doping in $\mathrm{MoS}_{2}$ whereas FePc and $\mathrm{CuPc}$ result in $p$-type doping. ${ }^{89}$ Interestingly, the charge-carrier density in hybrid MPc/TMD systems depends on the work function of the MPc metal centre, which can therefore be chosen ad hoc for achieving the desired level of doping. It is worth noting that $\mathrm{MPc} / \mathrm{MoS}_{2}$ FETs were found to be stable for at least two weeks after doping, despite the lack of any encapsulation layer. Finally, physisorbed MPc molecules have been investigated for 
passivating electronic defects, as well as for engineering surface states in defective monolayer $\mathrm{MoS}_{2}{ }^{217}$

The effects of organic dyes on the optoelectronic properties of TMDs were recently explored. ${ }^{218,229}$ In particular, perylenediimides and tetraphenyl porphyrins allowed increasing the conductivity of bottom-contact back-gate photodetectors based on relatively thick $\mathrm{MoS}_{2}$ sheets (approximately 10-layers) ${ }^{218}$ Such an increase was ascribed to the $n$-type doping induced by physisorbed molecules.

All these results demonstrate the possibility to control the charge-carrier doping in TMDs by molecular physisorption. However, further research is required to improve the stability of the charge transport properties in hybrid TMD-molecule systems, e.g. by developing suitable encapsulation techniques to prevent desorption or eventual decomposition of molecules during device operation and storage. Towards this goal, novel approaches are being explored. For instance, $\mathrm{Yu}$ et al. ${ }^{215}$ reported on the use of mixtures of poly(methyl methacrylate) (PMMA) and $\mathrm{F}_{4}$-TCNQ for simultaneously doping and encapsulating few-layer $\mathrm{WSe}_{2}$ FETs.

\subsection{Molecular engineering of the top and bottom TMD surface}

In this section, we highlight how molecular decoration enables significant engineering of TMDs also in the absence of strong covalent interactions and significant charge transfer. When organized in continuous and homogeneous layers, even inert molecules can modify the TMD optoelectronic properties, by acting as passivating/encapsulating agents ${ }^{230}$ or by interacting through (ordered) dipolar fields. ${ }^{39}$

In particular, the bottom TMD surface has been modified through a highly controllable molecular tuning of the physico-chemical properties of the substrate surface. This goal was achieved by inserting polymers or chemisorbed SAMs between the substrate and the TMDs. Instead, the top TMD surface is affected by the presence of highly ordered physisorbed layers 
composed of functional molecules. We highlight that whenever ordered monolayers are employed, the collective interaction between molecules and 2D materials is determined by the nanoscale molecular ordering. Precise control over the molecular arrangement at the nanoscale can thereof be exploited to finely tune the TMD optoelectronic properties. In this regard, the electrical and optical characterization of TMD/molecules systems should be ideally complemented by scanning tunnelling microscopy (STM) investigations, which offers the possibility to explore molecular adsorption with sub-molecular structural and functional resolution. ${ }^{231-234}$ In this way, STM could provide precious insights into the molecular-scale mechanisms leading to macroscopic effects, permitting not only to control the nanoscale assembly, but also to guide in the program and design of molecular units exhibiting the desired cooperative or collective effects. This possibility will be discussed in more detail in the conclusion section.

TMDs on polymers and functional SAMs. Polymers have often been employed as dielectric substrates for TMDs, ${ }^{205,206,235-237}$ in view of their mechanical flexibility that makes them compatible with bendable and stretchable electronics. ${ }^{47,238,239}$ As compared to conventional $\mathrm{SiO}_{2}$, dielectric polymer films can be remarkably different in terms of surface roughness, dielectric permittivity and phonon vibrations. ${ }^{205,240,241}$ Molecular design can be exploited to tailor the physico-chemical properties of polymeric surfaces, e.g. enabling a precise tuning of the surface energy. ${ }^{242}$ Hereafter, we present a few examples in which polymer-modified substrates result in a significant improvement of the (opto)electronic properties of TMDs.

Fig. 7a displays the general scheme of a typical FET based on TMD sheets deposited onto a dielectric polymer substrate. By using this architecture, Chamlagain et al. ${ }^{205}$ demonstrated that the room-temperature field-effect mobility of multilayer (5-15 nm thick) $\mathrm{MoSe}_{2}$ FETs on parylene $\left(100-150 \mathrm{cmV}^{-1} \mathrm{~s}^{-1}\right)$ was systematically higher than in the case of equivalent devices on $\mathrm{SiO}_{2}\left(\sim 50 \mathrm{cmV}^{-1} \mathrm{~s}^{-1}\right.$, Fig. $\left.7 \mathrm{~b}\right)$. The authors attributed such substrate dependent mobility to 
different phonon vibrations at the $\mathrm{SiO}_{2}$ and parylene surfaces. In particular, the scattering with polar optical phonons originating from the substrate limits the mobility of $\mathrm{MoSe}_{2}$ on $\mathrm{SiO}_{2}$, while it is nearly absent for $\mathrm{MoSe}_{2}$ on parylene. ${ }^{205}$ Alternative explanations for the measured effects include the polymer-mediated passivation of charged impurities on the $\mathrm{SiO}_{2}$ surface and a partial leveraging of the TMD layer due to the polymer roughness, which helps the removal of adsorbate molecules trapped below the bottom TMD surface during vacuum annealing. Similarly, the electronic performances of multilayer $\mathrm{MoS}_{2}$ on PMMA were found to be higher than those on $\mathrm{SiO}_{2}$. Remarkably, in the case of $\mathrm{MoS}_{2}$ on PMMA not only high $n$ type mobility was measured (up to $\sim 470 \mathrm{~cm}^{2} / \mathrm{Vs}$ ) but also $p$-type mobility (up to $\sim 480$ $\mathrm{cm}^{2} \mathrm{~V}^{-1} \mathrm{~s}^{-1}$ ), resulting in almost ideal ambipolar characteristics. The different electronic behavior of $\mathrm{MoS}_{2}$ on $\mathrm{SiO}_{2}$ and PMMA was attributed to short range disorder at the TMD/SiO 2 interface (e.g. roughness scattering). ${ }^{236}$

In a recent study, Liu et al. ${ }^{206}$ found that the PL spectrum of $\mathrm{MoS}_{2}$ onto the fluoropolymer CYTOP closely resembles that of self-standing $\mathrm{MoS}_{2}$ with a narrow and strong PL peak at the region of A exciton and without trion peak (Fig. 7c). A similar effect was observed for $\mathrm{WS}_{2}{ }^{206}$ FETs fabricated on $\mathrm{MoS}_{2}$ monolayers on CYTOP also showed lower intrinsic $n$-type doping and higher mobility than those fabricated on $\mathrm{SiO}_{2}$. In view of these results, the authors conclude that CYTOP is an ideal substrate for TMDs, on account of its very low surface energy, low surface trap densities and low permittivity. By taking full advantage of the polymeric substrate, the authors demonstrate a giant bandgap renormalization in back-gated $\mathrm{MoS}_{2}$ and $\mathrm{WS}_{2}$, which manifests itself as a sizeable gate-induced modulation of PL and differential reflectance (Fig. 7d). ${ }^{206}$

These examples show how TMD/substrate interactions, which remarkably influence the charge-transport properties of TMDs, ${ }^{243}$ can be tuned by making use of polymeric substrates, thereby offering a valuable strategy to engineer TMD-based devices. 
Similarly, the physico-chemical properties of the substrate can be tailored by using functional SAMs chemisorbed on solid substrates. ${ }^{244}$ The molecules employed to generate such architectures possess (i) an anchoring group that promotes the chemisorption onto the substrate, (ii) a molecular backbone that stabilizes the packing via side-to-side molecular interactions, and (iii) a functional headgroup that ultimately determines the physico-chemical characteristics of the exposed surface. ${ }^{245}$ TMDs can be deposited onto SAM-modified surfaces (e.g. Fig. 8a) and they display different properties depending on the molecular headgroups in the SAM. We point out that while SAMs are covalently bound to the substrate, TMD sheets are physisorbed onto SAMs and their interaction is mediated by relatively weak van der Waals forces. Crucially, non-covalent interactions among adjacent molecules forming the SAM endow high crystalline order with molecules oriented edge-on with respect to the substrate plane. ${ }^{244}$ In this way, the ordered nanoscale arrangement ensures that singlemolecule dipoles sum up, collectively generating significant electric fields. Therefore, when TMDs are exfoliated onto SAM-decorated substrates, they experience an electric field effect analogous to that generated by a constant non-zero gate voltage. As a result, highly ordered SAMs of high-dipole molecules can introduce significant shifts in the Fermi level of TMDs, ${ }^{84}$ resulting in doping effects that are mediated by purely electrostatic interaction, even in the absence of significant charge transfer. The two doping mechanisms - i.e. charge transfer ${ }^{82,87}$ or dipolar interactions ${ }^{87,246}$ - are often concomitant, and it is not always possible to completely disentangle one from the other. ${ }^{87,92}$

Experimentally, the possibility to modulate the charge carrier concentration via SAMs was demonstrated in a study of the optoelectronic characteristics of mechanically-exfoliated $\mathrm{MoS}_{2}$ sheets deposited onto different SAMs. ${ }^{84}$ The authors modified the $\mathrm{SiO}_{2}$ substrate employing SAMs composed of alkanes exposing an anchoring silane group and different functional headgroups. In particular, SAMs of three silane agents characterized by different dipole 
moments and polarities were employed: octyltrichlorosilane (OTS, $\left.\mathrm{CH}_{3}-\mathrm{SAM}\right)$, 3(trimethoxysilyl)-1-propanamine (APTES, $\left.\mathrm{NH}_{2}-\mathrm{SAM}\right)$, and trichloro- $(1 \mathrm{H}, 1 \mathrm{H}, 2 \mathrm{H}, 2 \mathrm{H}-$ perfluorooctyl)silane (FOTS, $\mathrm{CF}_{3}-\mathrm{SAM}$ ), as shown in Fig. 8a. The arrangement of the different molecules, which plays a fundamental role in the determination of the measured effects, was indirectly inferred on the basis of the surface energy of the SAMs, as measured by water contact angle. The effect of the different molecular dipoles on the optoelectronic properties of $\mathrm{MoS}_{2}$ was investigated by a combination of Raman spectroscopy, FET fabrication and characterization, as well as Kelvin probe force microscopy (KPFM) measurements. Fig. 8b shows the electrical characterization of FETs based on a few-layers $\mathrm{MoS}_{2}$ fabricated on the different SAMs. The FOTS SAM was found to induce a positive shift of the threshold voltage, i.e. it introduces $p$-type doping. On the other hand, the APTES SAM was found to introduce a shift of the threshold voltage towards more negative gate values, i.e. introduce $n$-type doping. Through KPFM, the authors showed that the position of the Fermi level of monolayer $\mathrm{MoS}_{2}$ could be modulated in a range of more than $0.45 \mathrm{eV}$ by the electrostatic interaction with FOTS and APTES. Finally, minor effects on the threshold voltage were observed for the OTS SAM as compared to the bare $\mathrm{SiO}_{2}$ substrate, indicating minor doping. ${ }^{84}$ These results were explained on the basis of the different orientation of the (well-aligned) molecular dipoles, which point towards opposite directions for FOTS and APTES. Instead, the relatively low dipolar moment of $\mathrm{CH}_{3}$ did not induce significant doping on $\mathrm{MoS}_{2}$. Nevertheless, even alkyl SAMs have interesting effects on the optoelectronic properties of TMDs. Recently, Ajayi et al. ${ }^{230}$ measured an ultranarrow low-temperature PL linewidth in $\mathrm{MoSe}_{2}$ (approaching the intrinsic limit) thanks to an effective substrate passivation achieved via alkyl SAMs. In particular, a 6-meV-wide PL peak was measured for $\mathrm{MoSe}_{2}$ on the alkyl SAM, in contrast to the broader 9-meV peak of $\mathrm{MoSe}_{2}$ on $\mathrm{SiO}_{2}$. Additionally, the presence of the alkyl SAM also affected the optical characteristics of $\mathrm{MoSe}_{2}$ 
encapsulated in $\mathrm{BN}\left(\mathrm{BN} / \mathrm{MoSe}_{2} / \mathrm{BN}\right)$, which exhibited broader PL on the bare $\mathrm{SiO}_{2}(3 \mathrm{meV})$ rather on the SAM-passivated surface $(2 \mathrm{meV})$.

Theoretically, it was predicted that the presence of SAMs exposing a polar headgroup (both $\mathrm{CH}_{3}$ and $\left.-\mathrm{CF}_{3}\right)$ anchored onto metal electrodes could promote the charge injection into $\mathrm{MoSe}_{2}$, converting Schottky metal-semiconductor junctions into nearly Ohmic contacts. ${ }^{95}$ These studies show how SAM engineering represents a straightforward approach to control the charge-carrier polarity enabling the fabrication of $p$ - $n$ junctions and complementary logic devices.

Taking a step further, a thorough choice of the molecules composing the SAMs makes it possible to confer unique SAM-derived capabilities to TMDs. This possibility was demonstrated by depositing $\mathrm{MoS}_{2}$ onto a photoswitchable SAM composed of a thiolated photochromic azobenzene (AZO) moiety ((E)-6-(4-(phenyldia- zenyl) phenoxy)hexane-1-thiol, HS-C 6 AZO) chemisorbed on a gold substrate (Fig. 8c). ${ }^{222,}{ }^{247}$ AZO are photochromic molecules which can be switched between a trans and a cis isomer upon light irradiation at different wavelengths (Fig. 8c). ${ }^{248}$ When AZO molecules self-assemble forming ordered chemisorbed monolayers on metals, the photoswitch is accompanied by a modification of the surface energetics, ${ }^{249-251}$ which is in turn reflected by a change of the optical properties of $\mathrm{MoS}_{2}$ monolayers. ${ }^{222}$ In particular, lower (higher) PL intensity was measure when the AZO-SAM was in the trans (cis) state, which was explained in terms of different molecule-induced doping in the two cases (Fig. 8d).

The same Au/AZO-SAM/MoS 2 stack was exploited to demonstrate photo-switchable vertical diodes, in which the current flowing vertically through the stack was measured by contacting the $\mathrm{MoS}_{2}$ sheet with a conductive tip in an atomic force microscope (AFM). With the AZOSAM in the trans state, a rectifying diode-like trace was measured with turn-on voltage close to $0.5 \mathrm{~V}$ (Fig. 8e). After photo-isomerization to the $c i$ state, the $I-V$ traces showed a 
suppression of the rectification, while the diode-like characteristics could be partially recovered by switching back the AZO-SAM to the trans state. These experiments demonstrate the possibility to modify reversibly the energetics of TMDs thanks to a unique molecular capability, such as the switching of photochromic molecules. In this regard, hybrid systems composed of functional molecules/TMDs represent an entirely new class of materials characterized by unique properties different from those of the isolated components even in the absence of covalent bonds.

Physisorbed crystalline monolayers on TMDs. While in the previous section we have discussed the case of molecular tailoring of the bottom TMD surface, here we focus on the effect of non-covalent molecular modification of the top TMD surface. Supramolecular interactions, in particular of van der Waals type, can drive the formation of physisorbed crystalline monolayers of molecules onto TMDs, in which the molecular dipoles self-align, ${ }^{81}$ 252,253 thereby giving rise to electrostatic effects analogous to those discussed in the previous section. This possibility was demonstrated by the works by Kang et al., ${ }^{39,86}$ in which ordered molecular layers were physisorbed onto the top TMD surface to induce non-degenerate doping effects (Fig. 9a). In contrast to the case of TMD onto SAM-modified substrates, in the case of top surface modification it was possible to measure the electrical characteristics of the TMDs before and after the formation of the molecular layer, enabling a precise estimation of the doping introduced by the molecular layer. Interestingly, the molecules investigated for the modification of the top surface of few-layer $\mathrm{MoS}_{2}$ and $\mathrm{WSe}_{2}$ (ODTS and APTES, Fig. 9a) bear the same functional groups used for tailoring the bottom substrate via SAM chemisorption (Fig. 8a). ${ }^{39,86}$ Fig. 9b displays the effect of ultrathin ODTS layer on the electrical characteristics of $\mathrm{WSe}_{2}$. In this case, a relatively small shift of the threshold voltage towards more positive values was measured, indicative of $p$-type doping. An analogous $p$-type doping was observed for ODTS onto $\mathrm{MoS}_{2}$, while the layer of APTES molecules was 
observed to induce $n$-type doping on both $\mathrm{WSe}_{2}$ and $\mathrm{MoS}_{2}$ (Fig. 9c). These results are qualitatively similar to those obtained for the same molecules at the bottom of TMDs, and similarly were explained by considering that the doping effects were mediated by molecular dipoles, which effectively changed the surface energetics. ${ }^{86}$ In particular, it was proposed that the molecular assemblies on the top and at the bottom are specular, so that the TMD layer is effectively affected by analogous electric fields.

We would like to point out that the molecular dipoles orientation is dictated by the molecular arrangement. The fact that analogous doping effects are measured also on different TMDs $\left(\mathrm{MoS}_{2}\right.$ and $\mathrm{WSe}_{2}$, Fig. 9c) indicates that the arrangement of a given molecule on the subnanometre scale on different vdW surfaces is the same. Indeed, analogous van der Waals forces drive the formation of the same assembly on the inert, chemically similar van der Waals surfaces of layered materials. ${ }^{252,253}$ Therefore, one can assume that the doping induced by the assembly of the same molecule on different TMDs is analogous, since the assembly is typically the same. ${ }^{252}$

We mention that the presence of a molecular film on the surface of TMDs can be exploited not only to modify the properties of TMDs at a fundamental level, but also to enable technologically relevant application. As an example, in the work discussed in Fig. 9a-c, it was found that the molecules improved the photoresponsivity and detectivity of TMD-based photodetectors. ${ }^{39}$ Moreover, highly ordered molecular monolayers adsorbed either above or below TMDs have been employed in combination with ultrathin inorganic layers as ultrathin gate dielectrics for flexible electronics. ${ }^{91,254}$ The resulting transistors operating at low voltage showed remarkable subthreshold swing $(<80 \mathrm{mV} / \mathrm{dec})$, limited hysteresis and high breakdown field. 


\section{Chemical treatments for optimizing optical processes in 2D TMDs}

The modifications in the optical properties of TMDs discussed in Section 2 arise from changes in the charge-carrier density induced by molecular layers. ${ }^{102,103,209,255}$ Here, we discuss a few recent experiments showing how appropriate chemical treatments can be used to optimize the photoluminescence (PL) emission in 2D TMDs in a way that cannot be explained by electronic doping, and which involves the passivation of surface defects.

Typically, the doping-induced PL modulation is relatively weak for monolayer $\mathrm{MoS}_{2}$, with $p$ dopants introducing up to a three-fold PL increase. ${ }^{249}$ In contrast, Amani et $a l .{ }^{97}$ reported on a two-orders-of-magnitude increase in the PL of chemically-treated $\mathrm{MoS}_{2}$, reaching near-unity quantum yield. In particular, the authors demonstrated that a treatment with the nonoxidizing organic superacid bis(trifluoromethane)-sulfonimide (TFSI, inset in Fig. 10a) determined an up-to-190-fold increase in the PL intensity without significant changes in the spectral shape (Fig. 10a). PL maps of the same 2D $\mathrm{MoS}_{2}$ sheet measured before and after the superacid treatment revealed that the increase was spatially homogeneous (Fig. 10b). Remarkably, it was found that at low excitation intensity $\left(<10^{-2} \mathrm{Wcm}^{-2}\right)$, the PL quantum yield approached $\sim$, i.e. almost the totality of the incoming photons were re-emitted (Fig. 10c). Following the discussion in Section 2, one might expect the PL boost to be mediated by $p$ doping, since TFSI is a strong electron acceptor. ${ }^{256}$ Through the comparative electrical characterization of a $2 \mathrm{D}-\mathrm{MoS}_{2}$ FET before and after the superacid treatment, the authors demonstrated that TFSI would not induce significant $p$-type doping. Instead, the passivation of surface defects was put forward as main effect leading to the increased PL. ${ }^{97}$ Indeed, the low PL quantum yield of (untreated) $\mathrm{MoS}_{2}$ at low excitation power is typically attributed to defect mediated non-radiative recombination; ${ }^{70}$ hence the near-unity quantum yield of the treated samples indicates a defect passivation. However, the exact mechanism through which defects are passivated is not entirely understood. More recent studies have shown that the 
same superacid treatment could be successfully applied to CVD-grown $\mathrm{MoS}_{2},{ }^{98}$ and that its effect could be made stable by encapsulation with a CYTOP film. ${ }^{257}$ Moreover, it was demonstrated that not only the PL of $\mathrm{MoS}_{2}$ but also that of $\mathrm{WS}_{2}$ is boosted by the TSFI treatment. ${ }^{96}$ On the contrary, the TSFI treatment does not enhance the quantum yield of Sebased TMDs, such as $\mathrm{WSe}_{2}$ and $\mathrm{MoSe}_{2} \cdot{ }^{96}$ An STM investigation of the defects in sulfide- and selenide-based TMDs suggested that the different effects of the TSFI treatment could be explained by the distinct nature of the defects in TMD selenides and sulfides. ${ }^{96}$ In particular, the defects found on sulfide surfaces were either structural defects or acceptor impurities, whereas those found on selenide surfaces were mainly donor impurities. ${ }^{96}$

Similarly to the case of TFSI, an acid-mediated sulfur vacancies self-healing was put forward in a recent experiment in which $\mathrm{MoS}_{2}$ was placed in contact with non-oxidizing acid poly(4styrenesulfonate) (PSS). ${ }^{258}$ In this case, self-healing was accompanied by a $p$-type doping effect and a rather limited PL increase (two-fold).

A similar acid based self-healing of defects was demonstrated for Se-based TMDs. In particular, it was demonstrated that the PL of CVD-grown $\mathrm{MoSe}_{2}$ could be increased via treatment with hydracids. ${ }^{100}$ The highest effect was found for hydrohalic acid ( $\left.\mathrm{HBr}\right)$, which caused a 30-fold increase in the PL of $\mathrm{MoSe}_{2}$ (see Fig. 10d-e). In this case, two concomitant mechanisms were identified to account for the measured effect: (i) an induced $p$-type doping, as inferred from a Raman characterization and (ii) an acid-mediated structural healing, inferred on the basis of low temperature PL spectra and XPS characterization. In particular, for untreated $\mathrm{MoSe}_{2}$ sheets, the PL spectrum was dominated by a rather broad PL peak at $\sim 1.56 \mathrm{eV}$, which was ascribed to the radiative emission of excitons bound to defects (Fig. 10f). Instead, after $\mathrm{HBr}$ treatment, the low temperature PL became more structured, with two sharper peaks corresponding to the neutral exciton and trion peak. The decrease in the relative intensity of the defect-related peak indicated the $\mathrm{HBr}$-induced defect healing in $\mathrm{MoSe}_{2}$. In 
particular, the authors found spectroscopic evidences of undesired oxidized Se in the asgrown CVD $\mathrm{MoSe}_{2}$, and concluded that $\mathrm{HBr}$ effectively passivates these defects by replacing oxidized Se defects $(\mathrm{Se}-\mathrm{O})$ with $\mathrm{Br}$, which covalently binds to Mo atoms. ${ }^{100}$

These experiments indicate the potential of acid treatments to passivate defect-mediated recombination pathways in TMDs, leading to almost-ideal optical properties. Therefore, the chemically treated ultrathin TMDs might enable the demonstration of optimized optoelectronic devices, such as high-performance light-emitting diodes, lasers and solar cells.

\section{Covalent functionalization via defect and phase engineering}

During the last five years, several works have been published on the covalent functionalization of ultrathin TMDs, mainly focusing on solution-processed $\mathrm{MoS}_{2}$ nanosheets produced via ion intercalation $\left(1 \mathrm{~T} / 1 \mathrm{~T}^{\prime} \text { phase }\right)^{259,260}$ or ultrasonication $(1 \mathrm{H} / 2 \mathrm{H})$ methods. ${ }^{110,261}$ These studies will be presented in Section 5, which is dedicated to solution-based approaches for the preparation of inks of functionalized TMDs. Here, we will review those investigations carried out on substrate-supported nanosheets, which aimed at "decorating" the top surface of the $2 \mathrm{D}$ materials with molecules through bond-formation processes. As mentioned in the introduction, $\mathrm{MoS}_{2}$ is the prototypical element of the TMD class, ${ }^{19}$ so that pioneering explorations in this research area have been carried out mostly on this promising 2D semiconductor. However, the concepts and strategies described in the following can be easily extended to other ultrathin TMDs, in particular MoWSeS materials, which have structural and physico-chemical properties similar to those of $\mathrm{MoS}_{2}$. Indeed, all MoWSeS crystals exhibit a stable $1 \mathrm{H} / 2 \mathrm{H}$ semiconducting phase ${ }^{9,150}$ and their surface lacks dangling bonds, a fact that makes the covalent functionalization of the basal plane a challenging objective. ${ }^{80,110,200,260}$ In 2014, Azcatl et al. ${ }^{262}$ reported on a non-disruptive method for the functionalization of $\mathrm{MoS}_{2}$ based on exposure to UV-ozone. The latter induces the formation of an oxygen layer on 
the surface of the $2 \mathrm{D}$ semiconductor, which can be conveniently exploited as a seed layer for the growth of high-quality dielectric films by atomic layer deposition (ALD). ${ }^{262,}{ }^{263}$ Noticeably, the structural and the electronic properties of $\mathrm{MoS}_{2}$ were not found to be altered by the UV-ozone treatment, which supported the finding of weak bonding interactions between oxygen and sulfur atoms. ${ }^{262,263}$

Nowadays, two major strategies are being explored towards the strong covalent or coordinative bonds between TMDs and atomic or molecular adlayers. The first involves the activation/optimization of the chemical reactivity of the nanosheets' surface via the controlled generation of point defects, such as chalcogen vacancies. In such a way, dangling bonds are generated locally at defect sites, enabling the formation of chemical bonds between the defective surface and molecules with ad hoc functional groups. The second method deals with the phase tunability of TMDs, ${ }^{135}$ which allows for the reversible conversion of the electronrich and easy-to-functionalize polytype $\left(1 \mathrm{~T} / 1 \mathrm{~T}^{\prime}\right)$ into the more inert semiconducting polytype $(1 \mathrm{H} / 2 \mathrm{H}) .{ }^{80}$ As we shall see, the latter approach enables preparing functionalized $1 \mathrm{H} / 2 \mathrm{H}$ nanosheets through phase-conversion processes triggered by chemical or thermal stimuli. ${ }^{80}$

\subsection{Engineering chemically-active defects}

Numerous techniques have been used to engineer defects, such as chalcogen vacancies, in the basal plane of ultrathin TMDs, among which electron irradiation, ${ }^{264-267}$ thermal annealing, ${ }^{88,}$ 268, 269 electrochemical generation, ${ }^{186}$ plasma treatments, ${ }^{185,}, 270-273$ as well as physical bombardments with charged particles, including ions of argon, ${ }^{109,}$ 274-276 helium, ${ }^{277-279}$ manganese, ${ }^{280} \alpha$ particles $\left(\mathrm{He}^{2+}\right)^{159}$ and swift heavy ions $(e . g . \mathrm{Xe} / \mathrm{Ta}, E \approx 80-100 \mathrm{MeV}) .{ }^{281}$ Moreover, chalcogen vacancies have been systematically generated during the growth/synthesis process via non-equilibrium CVD methods, ${ }^{223}$ as well as through hydrothermal synthetic strategies. ${ }^{282}$ Theoretical calculations have also been carried out to corroborate experimental results and elucidate the structures/properties of the defects 
produced with such techniques, e.g. in the case of electron irradiation (refs 68, 267) and ion bombardment (ref. 283). In 2012, Komsa et al. ${ }^{68}$ carried out a joint theoretical and experimental investigation on the effects of electron irradiation on the atomic structure of ultrathin TMDs. In particular, they observed the formation of sulfur vacancies (SV) in $1 \mathrm{H}-$ $\mathrm{MoS}_{2}$ sheets exposed to an electron beam $(E \approx 80 \mathrm{keV})$ within a high-resolution transmission electron microscope (HRTEM). The possibility of filling the SVs with donor and acceptor impurities - with the objective of introducing doping - was supported by calculations of the formation energy of substitutional defects. Experimentally, in situ HR-TEM studies provided evidence for the occurrence of SV filling (see Fig. 11a-c), though it was not possible to identify the type of the impurity involved in the process. Subsequent theoretical works were carried out on substitutional doping of TMDs, and various strategies have been proposed for adding novel functionalities to $\mathrm{MoS}_{2}$ by means of magnetic, rare-earth and chalcogen dopants, as well as molecular ions. ${ }^{284,285}$ More recently, a combined TEM and Raman-spectroscopy study (ref. 265) allowed establishing a correlation between the density of SVs in electronirradiated $\mathrm{MoS}_{2}$ nanosheets and the energies of the main Raman-active phonon modes $\left(E_{2 \mathrm{~g}}^{1}\right.$ and $A_{1 \mathrm{~g}}$ ), thereby providing an indirect means for quantifying SVs in ultrathin $\mathrm{MoS}_{2}$.

In 2013, Ma et al. ${ }^{274}$ showed that low-energy $(E \approx 500 \mathrm{eV})$ argon-ion irradiation of monolayer $\mathrm{MoS}_{2}$ results in the controlled generation of SVs, as evidenced by X-ray photoemission spectroscopy (XPS) studies, see Fig. 11d. In contrast to their electron counterpart, ion beams have relatively larger diameters $(\sim 100 \mathrm{~s} \mu \mathrm{m})$ and can be rastered over centimetre-scale large areas in relatively short time, enabling the fast processing of wafer-scale CVD-grown films. Although this was the first study on a truly 2D semiconductor, it should be mentioned that several investigations have been carried out in the past on the effects of argon ion bombardment on bulk TMD crystals. ${ }^{187,286-291}$ In 1972, Williams et al. ${ }^{286}$ performed lowenergy $(E \approx 300 \mathrm{eV})$ argon-ion bombardment on the surface of various layered chalcogenide 
materials, among which $\mathrm{MoS}_{2}, \mathrm{NbSe}_{2}, \mathrm{ZrS}_{2}, \mathrm{MoTe}_{2}, \mathrm{TiTe}_{2}$ and $\mathrm{SnS}_{2}$, revealing - in most of the cases - a significant enhancement of the reactivity of the basal plane in the presence of oxygen species. This was attributed to the pitting and faceting of the surface upon ion bombardment, leading to the increase of the effective surface area of the sample. ${ }^{286}$ However, little was known about the structure of the defects. Two years later, Feng and Chen ${ }^{287}$ conducted XPS measurements on bulk $\mathrm{MoS}_{2}$ crystals irradiated with argon ions of $\sim 300 \mathrm{eV}$, and observed the formation of islands of metallic molybdenum along with a decrease in the sulfur content. The authors hypothesized that sulfur atoms, which have a mass similar to that of argon, could be ejected more efficiently in comparison to heavier molybdenum atoms, thereby enabling a selective sputtering process. Nowadays, bombardment with argon-ion beams is a common and established method for engineering chalcogen vacancies in ultrathin TMDs, ${ }^{274} 109,275$ together with similar techniques based on plasmas of argon ions. ${ }^{185,273}$ In 2016, Li et al. ${ }^{185}$ showed - both theoretically and experimentally - that SVs generated via argon plasmas lead to the activation of the basal plane of $1 \mathrm{H}-\mathrm{MoS}_{2}$ monolayers for hydrogen evolution reaction (HER), which could be further optimized through the application of inplane strain. The HER activity of $1 \mathrm{H}-\mathrm{MoS}_{2}$ sheets has also been enhanced through electrochemical desulfurization processes (ref. 186), which allowed tuning the density of SVs by changing the applied desulfurization potential. Additionally, complementary thermal approaches have been developed for the generation of SVs, such as annealing in ultra-high vacuum $\left(p \approx 10^{-9} \text { torr, } T \geq 200^{\circ} \mathrm{C}\right)^{268}$ or in $\operatorname{air}\left(T \approx 250{ }^{\circ} \mathrm{C}\right) .{ }^{88}$

Having a high density of chemically-active chalcogen vacancies is desired for the purpose of functionalization, but it can also be very detrimental in other perspectives, e.g. for applications in (opto)electronic devices. In a recent work, the effects of ion-beam induced SVs on the optical, vibrational, as well charge transport properties of mechanically-exfoliated monolayer $\mathrm{MoS}_{2}$ have been investigated (ref. 109). The unencapsulated channel of back-gated 
monolayer FETs has been irradiated with low-energy $(E \approx 500 \mathrm{eV})$ argon ions, as shown in Fig. 11e. This allowed monitoring the evolution of the electrical characteristic of the devices with increasing ion dose, as shown in Fig. $11 \mathrm{f}$ and g. Noticeably, the experiments were carried out under inert atmosphere to minimize possible oxygen chemisorption at defect sites, which could affect the electronic structures/properties of semiconducting TMDs. ${ }^{292}$ The results reported in this work ${ }^{109}$ provided a guideline for the trade-off choice between density of SVs suitable for chemical functionalization and device performance, in terms for instance of charge-carrier mobility and $I_{\mathrm{on}} / I_{\mathrm{off}}$ switching ratio in FETs.

\subsection{Repair and functionalization of defective TMDs}

In the last few years, various research groups have explored the possibility of repairing/functionalizing defective $\mathrm{MoS}_{2}$ nanosheets with small organic molecules carrying thiol headgroups. ${ }^{88,109,293}$ Noticeably, the reactivity of SVs with thiol molecules has been investigated since the 1970 s, mainly due to the widespread use of $\mathrm{MoS}_{2}$ as a catalyst for hydrodesulfurization to remove sulfur from oil. ${ }^{294}$ The adsorption and decomposition of alkanethiols on defective $\mathrm{MoS}_{2}$ surfaces have been previously investigated, for instance in the case of butanethiol, ${ }^{294}$ ethanethiol, $^{295}$ methanethiol $^{296}$ and dodecanethiol. ${ }^{181}$ In 2012 , Makarova et al. ${ }^{181}$ reported a STM study supporting the hypothesis of bond formation between thiol groups and unsaturated molybdenum atoms at SV sites. In particular, two thiol derivatives - namely (3-mercaptopropyl)-trimethoxysilane (MPS) and dodecanethiol - were adsorbed to the surface of bulk $2 \mathrm{H}-\mathrm{MoS}_{2}$ crystals, as schematized in Fig. 12a. The density of molecules was found to match the typical density of $\mathrm{SVs}$ in common $\mathrm{MoS}_{2}$ samples, suggesting a possible chemisorption of the thiol molecules at SV sites. Interestingly, the authors observed that both thiol derivatives could be easily desorbed from the surface by applying electrical stimuli with the STM tip, a phenomenon that was ascribed to the tipinduced dissociation of the S-C bond (Fig. 12b). Remarkably, such a process was found to 
lead to the filling/repairing of the SVs. Inspired by these results, Yu et al. ${ }^{182}$ developed a thiol-chemistry approach with the aim of healing defects and improving the charge-transport properties of ultrathin sheets of $\mathrm{MoS}_{2}$. The treatment consists in the immersion of $\mathrm{SiO}_{2} / \mathrm{Si}$ substrates carrying mechanically-exfoliated $\mathrm{MoS}_{2}$ flakes in a solution of MPS in dichloromethane, followed by thermal annealing $\left(T \approx 50{ }^{\circ} \mathrm{C}, 20 \mathrm{~min}\right)$ to induce the scission of the S-C bond and remove the excess MPS molecules. Density functional theory calculations (DFT) were carried out to assess the kinetics of the reaction, shown in Fig. 12c. The effective reduction of the number of SVs was supported by a statistical analysis of HR-TEM images acquired from as-exfoliated and treated samples, which revealed a decrease in the average SV density from $\sim 6 \times 10^{13} \mathrm{~cm}^{-2}$ to $\sim 1.6 \times 10^{13} \mathrm{~cm}^{-2}$. Thanks to their trimethoxysilane groups that chemisorb on $\mathrm{SiO}_{2}$, MPS molecules have also been used for the formation of SAMs on the oxidized silicon substrates. In this case, the molecules expose their thiol groups towards the overlying $\mathrm{MoS}_{2}$ nanosheets, thereby providing - upon annealing - an opportunity for healing SVs in the bottom surface. The effects of such thiol-chemistry treatments on the electrical properties of back-gated $\mathrm{MoS}_{2}$ FETs are reported in Fig. 12d and e. Upon repairing SVs in the top and bottom surfaces, the field-effect mobility increases up to $\sim 80 \mathrm{~cm}^{2} \mathrm{~V}^{-1} \mathrm{~s}^{-1}$ (at room temperature), which is one of the highest values achieved so far using back-gated monolayer $\mathrm{MoS}_{2}$ FETs on conventional $\mathrm{SiO}_{2} / \mathrm{Si}$ substrates. ${ }^{297} \mathrm{~A}$ follow-up work by Cho et al. ${ }^{293}$ focused on the passivation of SVs in multilayer $\mathrm{MoS}_{2}$ sheets $(2-11 \mathrm{~nm}$ thick) with alkanethiol molecules. The healing procedure, based on the immersion of the samples into an ethanol solution of hexadecanethiol (or octanethiol) molecules, did not include the annealing step previously used to promote the scission of the S-C bond and the removal of not-chemisorbed molecules. As a result, the electrical current and the charge-carrier mobility in FETs were found to decrease. This was ascribed to trap states associated with alkanethiol molecules chemisorbed at SVs. Sim et al. ${ }^{88}$ adopted a similar approach aiming at introducing a stable 
doping in few-layer $\mathrm{MoS}_{2}$ nanosheets (2-4 layer thick) via chemisorption of thiol molecules carrying donor or acceptor groups, see Fig. 12f. After immersing the samples in ethanol solutions of mercaptoethylamine $\left(\mathrm{NH}_{2}\right.$-terminated thiol, MEA) and $1 \mathrm{H}, 1 \mathrm{H}, 2 \mathrm{H}, 2 \mathrm{H}-$ perfluorodecanethiol $\left(\mathrm{CF}_{3}\right.$-terminated thiol, FDT), the authors performed a mild annealing under inert atmosphere $\left(100{ }^{\circ} \mathrm{C}, 30 \mathrm{~min}\right)$ and an abundant solvent rinsing to remove unbound molecules. Such a treatment resulted in $n$-doping in the case of MEA $\left(\Delta n \approx 3.7 \times 10^{12} \mathrm{~cm}^{-2}\right)$ and $p$-doping in the case of FDT $\left(\Delta n \approx-7.0 \times 10^{11} \mathrm{~cm}^{-2}\right)$, which was supported by multiple techniques, including PL spectroscopy, XPS and electrical measurements. In FETs, the doping effect appeared more evident after an additional annealing step $\left(T \approx 150{ }^{\circ} \mathrm{C}, 10 \mathrm{~min}\right)$ that served for removing water and oxygen molecules that are known to behave as electron acceptors. ${ }^{298}$ The resulting transfer characteristics of pristine (black) and doped (red and blue) few-layer $\mathrm{MoS}_{2}$ FETs are shown in Fig. 12g. More recently, short-chain alkanethiols, such as butanethiol, have also been used to heal SVs introduced via low-energy ion irradiation in the channel of monolayer $\mathrm{MoS}_{2}$ FETs (ref. 109). In this case, a vapour-phase deposition process — carried out strictly under inert atmosphere without other external influences - enabled a significant recovery of the electrical characteristics of ion-irradiated FETs, confirming the occurrence of defect healing by thiol molecules.

The results discussed so far showed that repair (involving the dissociation of the S-C bond) and functionalization of SVs via chemisorption of thiols are two possible outcomes. This aspect has been investigated theoretically by two groups, ${ }^{183,184}$ who showed by means of DFT calculations that both reactions can actually occur. Nevertheless, the mechanisms involved might vary depending not only temperature but also on polarization effects associated with the molecule's decorating groups. ${ }^{183}$ In the case of methanethiol, Förster et al. ${ }^{184}$ found that repair is energetically most favourable over functionalization, whereas Li et al. ${ }^{183}$ concluded that functionalization products are preferred due to slightly smaller energy barriers. However, it 
should be noticed that such discrepancies may arise from different assumptions on the role of hydrogen in the reaction (adsorption/release), which is a debated argument in the chemistry of thiols at metal surfaces. ${ }^{244,299-303}$ At this stage, more investigations are needed to identify the exact experimental conditions leading to the repair or functionalization of SVs.

The methods discussed above can be extended to other elements of the TMD family, in particular MoWSeS materials. For instance, the generation, healing and doping functionalization of selenium vacancies in ultrathin $\mathrm{WSe}_{2}$ have been already reported in the last years. ${ }^{79,271,304}$ It is expected that $2 \mathrm{D}$ transition metal selenides could be similarly functionalized/repaired with molecules carrying selenol headgroups,${ }^{305}$ or once again repaired with thiol molecules - since sulfur and selenium are valence-isoelectronic elements - and this would allow developing 2D nanosheets with tunable chalcogen composition (e.g. $\left.\mathrm{WS}_{2 \mathrm{x}} \mathrm{Se}_{2(1-\mathrm{x})}\right)^{306}$ Despite problems associated to the limited chemical stability of selenol exposing molecules, novel investigations can be foreseen to explore the viability of these approaches.

\subsection{Covalent functionalization via phase engineering}

An alternative strategy for the covalent functionalization of ultrathin $\mathrm{MoS}_{2}$ was reported in 2015 by Chhowalla and coworkers. ${ }^{80}$ Such a method does not involve chemical reactions at defects, as previously discussed, but it rather exploits the phase tunability of TMDs ${ }^{135}$ via chemical and thermal stimuli. It is known that chemical exfoliation with $n$-butyllithium leads to electron-rich nanosheets that contain $\sim 65 \%$ of the metallic $1 \mathrm{~T} / 1 \mathrm{~T}^{\prime}$ polytype. $^{141,142}$ Interestingly, also the exposure of substrate-supported CVD $\mathrm{MoS}_{2}$ sheets to $n$-butyllithium results in the change from the $1 \mathrm{H}$ to the $1 \mathrm{~T} / 1 \mathrm{~T}^{\prime}$ phase, as illustrated in Fig. 13b. This procedure was later shown to be also applicable to continuous polycrystalline $\mathrm{MoX}_{2}$ films. ${ }^{307}$ This can be performed in a spatially-controlled manner, which is suitable for patterning metallic regions in a semiconducting 2D layer to develop low-resistance contacts for ultrathin 
$\mathrm{MoS}_{2}$ FETs. ${ }^{308}$ Chhowalla et al. $^{80}$ showed that metallic $1 \mathrm{~T} / 1 \mathrm{~T}^{\prime}$ nanosheets can be easily functionalized by subjecting them to methyl iodide and iodoacetamide electrophiles (see Fig. 13a), resulting in functional groups that are covalently bound to sulfur atoms. Here, the reaction is promoted by charge transfer between the electron-rich $1 \mathrm{~T} / 1 \mathrm{~T}^{\prime} \mathrm{MoS}_{2}$ and the organohalides, which allows for achieving a degree of functionalization of the order of $\sim 30 \%$ (estimated by XPS). Atomic-resolution TEM studies carried out on functionalized nanosheets revealed that the original atomic structure with octahedral coordination is not altered by such a process (Fig. 13c). However, the functionalized 2D $\mathrm{MoS}_{2}$ displayed optical properties remarkably different from those of its not-functionalized counterpart, including fluorescence emission (Fig. 13d). The latter indicates the opening of a semiconducting bandgap, which is a promising result proving the possibility of tailoring ultrathin TMDs by the combination of chemical and phase-engineering approaches.

The authors further showed that CVD monolayer $\mathrm{MoS}_{2}$ films can be converted from $1 \mathrm{H}$ to $1 \mathrm{~T} / 1 \mathrm{~T}^{\prime}$ via $n$-butyllithium exposure; they can then be functionalized with electrophile molecules and subsequently re-converted into the $1 \mathrm{H}$ polytype by thermal annealing at $T \geq 300{ }^{\circ} \mathrm{C}$. Raman and XPS spectroscopy confirmed the occurrence of the semiconductingphase recovery, whereas attenuated total reflectance Fourier transform infrared (ATR-FTIR) spectroscopy indicated that functional groups were still present on the surface of the $1 \mathrm{H} \mathrm{MoS} 2$ sheets. This technique - based on a two-step phase conversion process - is being regarded as a promising strategy for the covalent functionalization of semiconducting MoWSeS materials, which otherwise could not be functionalized in their pristine form due to the lack of dangling bonds. It is worth noting that the methods described above were applied also to $\mathrm{WS}_{2}$ and $\mathrm{MoSe}_{2}$, and are expected to be applicable to other TMDs. ${ }^{76,80}$ In this research area, additional investigations are envisioned for tuning of the optical and electronic properties of ultrathin 
TMDs ad hoc for applications, which can be achieved for instance by a careful choice/design of the electrophile molecules.

\section{Functionalization of solution-processed TMD nanosheets}

Functionalization can also be achieved on TMD nanosheets in the liquid phase by a number of strategies. The prerequisite is an efficient exfoliation in liquid to obtain colloidally stable dispersions. For TMDs, two main strategies exist which are referred to as chemical exfoliation (CE) and liquid phase exfoliation (LPE), respectively. Chemical exfoliation is based on the intercalation chemistry of TMDs first described in the 1970s. ${ }^{117,309}$ Based on these early reports, the intercalation chemistry was explored in detail in the past century. ${ }^{310}$ The current understanding of the chemical exfoliation is described in detail elsewhere ${ }^{15,115}$ and is only briefly summarized here. The most commonly used technique is based on the reaction of bulk TMD powder with $n$-BuLi under inert conditions. Lithium is inserted between the layers followed by an electron transfer to the TMD resulting in an $\mathrm{Li}_{\mathrm{x}} \mathrm{MX}_{2}$ salt. Due to the Coulomb repulsion between the negatively charged layers, efficient exfoliation down to predominantly monolayers can be achieved after agitation/sonication in water. After purification (removal of excess $n$-BuLi, reaction side products, unexfoliated TMD), a colloidal suspension of negatively charged nanosheets in water is obtained. Due to the excess charge, the MoWSeSTMDs undergo a transition from the semiconducting $2 \mathrm{H}$ polytype (trigonal prismatic arrangement of chalcogen) to the metallic $1 \mathrm{~T} / 1 \mathrm{~T}^{\prime}$ polytype (octahedral arrangement of chalcogen, often distorted) ${ }^{115}$ - see Section 1.1. The metallic 1T-polytype is metastable and can be converted to the $2 \mathrm{H}$-polytype on annealing or by ageing which is usually accomplished by flocculation of the TMDs if this takes place in the dispersion. Depending on the reaction conditions, ${ }^{260,311,312}$ defective nanosheets (chalcogen vacancies and/or holes on the basal plane) or mixtures of the 1T/2H-polytype are accessible. 
A complementary technique that yields colloidally stable dispersions of group VI-TMDs in the semiconducting 2H-polytype is liquid phase exfoliation (LPE). As summarized in detail in recent reviews, ${ }^{106,313-317}$ this process involves sonication (or other forms of agitation such as shearing, ball milling) of the bulk powder in appropriate solvents or aqueous surfactant systems. A range of polymers (including biopolymers) can also be used as stabilizing agents in both aqueous and organic media. The energy or mechanical stress is required to overcome the van der Waals-type intersheet interaction and causes exfoliation of the bulk material into few-layers in the liquid with the nanosheet reaggregation prevented by the solvent/stabilizer. This process has first been demonstrated in 1989 via sonication of TMDs in water in an argon-hydrogen atmosphere. ${ }^{318}$ However, in ambient conditions, water as a neat solvent (i.e. without additional stabilizers) is not a suitable solvent to stabilize the TMD nanosheets. It was first demonstrated by Coleman et al. in $2011,{ }^{122}$ that solvents with solubility parameters matching the TMD can be used to obtain colloidally stable dispersions under ambient conditions. Suitable solubility parameters to describe the solution thermodynamics are solvent surface tension or energy, Hildebrandt parameters or Hansen parameters. Typical solvent choices are N-methyl-2-pyrrolidone, N-cyclohexyl-2-pyrrolidone, dimethyl formamide or isopropanol to a lesser extent. Alternatively, as first demonstrated by the same group, ${ }^{319}$ aqueous surfactant solutions can be used. The resultant nanosheet dispersion are highly polydisperse with respect to both layer number and lateral size, but size selection techniques have been developed to tackle this problem. ${ }^{320}$

Based on such nanosheet dispersions, numerous strategies have been developed to functionalize both basal plane and edges (Fig. 14). Due to the high surface area of the nanosheets, non-covalent functionalization via physisorption of various molecules, polymers (or noble metal nanoparticles) is commonly exploited. Note that numerous hybrid structures with other nanomaterials can be constructed in such a way as outlined elsewhere. ${ }^{321}$ The most 
widespread use of this non-covalent approach is to prevent nanosheets from reaggregation after LPE by adsorption of small molecules ${ }^{105,319,322-332}$ or (bio)polymers ${ }^{333-361}$ (see Table 2). The organic functional groups are believed to adsorb to the nanosheets via predominantly van der Waals interaction and dipole interactions can also occur. ${ }^{203,362}$

Similar to graphene, stabilization in the aqueous environment in the case of ionic surfactants is achieved mostly due to Coulomb repulsion that can be described within the DerjaguinLandau-Verwey-Overbeek (DLVO) theory, even though steric hindrance can also play an important role. ${ }^{363}$ Unfortunately, it is extremely challenging to unravel experimentally how the surfactant adsorption occurs, or indeed how densely the surfactant is packed on the surface. Ref. 326 is highlighted in this regard, as it provides evidence that surfactant chains of the cationic amphiphile cetyltrimethylammonium bromide (CTAB) and the anionic surfactant sodium dodecyl sulfate (SDS) lie flat, arranged randomly on the basal plane of the $\mathrm{MoS}_{2}$ nanosheets with their charged headgroup exposed. It was also shown that they exchange rapidly with free surfactants in the surrounding water. One of the most widely used classes of surfactants are facial amphiphiles such as sodium cholate (SC). ${ }^{105,319,324,327-329,} 331$ As demonstrated in the original work, ${ }^{319}$ such surfactant-based dispersions can be used to fabricate thin films or nanocomposites (e.g. by vacuum filtration) which can for example be used as electrodes in electrochemical water splitting. ${ }^{364,365}$ However, as shown recently, SC can have detrimental effects for electrocatalysis ${ }^{366}$ which emphasizes that more research is required to explore strategies to remove physisorbed molecules/polymers after processing for certain applications.

In the case of non-ionic surfactants ${ }^{332}$ or polymers, ${ }^{333-345}$ stabilization is due to steric effects that can be described in the framework of solution thermodynamics. ${ }^{334}$ In general, poloxamers (e.g. Pluronics) $335,339,341,344,345,367$ are the most widely studied polymeric stabilizers. In particular Pluronic F68 has been used to decrease the buoyant density of the nanosheet- 
polymer hybrid in water to allow for thickness sorting in a density gradient. ${ }^{367}$ Among the various structural characteristics, the thickness of the TMD sheets is known to define their properties; hence, the use of efficient layer number separation techniques is important. However, the yields of this process are currently low due to a low monolayer yield in the initial dispersion. ${ }^{367}$ This encouraged the authors to screen 19 different poloxamers with respect to their exfoliation efficiency (dispersed nanosheet concentration and monolayer content). ${ }^{341}$ Nevertheless, as outlined in ref. 335 , the presence of polymers during the exfoliation can result in poorer exfoliation compared to solvent or small molecule-assisted LPE in terms of mean layer number and lower dispersed concentration, as the surface tension of the liquid increases with the polymer concentration. The authors suggested to add the polymer stepwisely during the exfoliation to overcome this. ${ }^{335}$

In addition, a range of natural polymers/oligomers ${ }^{346-361}$ have been exploited in the noncovalent functionalization as summarized in Table 2. These are typically used to obtain biocompatible TMDs for biomedical ${ }^{198}$ and biosensing ${ }^{368}$ applications. Popular examples include chitosan, ${ }^{349,350,358,359}$ bovine serum albumin ${ }^{351,352}$ or oligonucleotide sequences. ${ }^{353,}$ 354, 360 The interaction with the TMDs can be considered as predominantly van der Waalstype, while the stabilization is based on both Coulomb and steric repulsion.

Another non-covalent functionalization strategy is based on the electrostatic interaction of cationic molecules with negatively charged 1T-TMDs. ${ }^{369-371}$ Such colloids are more stable than the $1 \mathrm{~T}-\mathrm{MX}_{2}$ in water on its $\mathrm{own}^{369}$ or can be used for a solvent exchange to organic media. ${ }^{370,371}$ Stabilization with oleylamine in high boiling point solvents such as octadecene or $o$-dichlorobenzene allows the conversion of $1 \mathrm{~T}-\mathrm{MX}_{2}$ to $2 \mathrm{H}-\mathrm{MX}_{2}$ in the dispersions by heating without causing flocculation. ${ }^{371}$

Even though the basal plane of TMDs is widely considered inert, a number of functionalization strategies via chemisorption have been developed. As illustrated in Fig. 14, 
these can be classified as (i) covalent (ii) coordinative, (iii) defect or (iv) coordinative defect functionalization. Note that not all strategies are accessible from both $1 \mathrm{~T}-\mathrm{CE}-\mathrm{MX}_{2}$ and $2 \mathrm{H}-$ LPE-MX ${ }_{2}$ or lead to a different result with respect to the degree of functionalization (number of functional groups per $\mathrm{MX}_{2}$ ) or type of binding as illustrated in Fig. 15 and summarized in Table 2-6.

Covalent modification refers to a reductive functionalization of negatively charged 1T-CE$\mathrm{MX}_{2}$ with electrophiles and involves the formation of a carbon-chalcogen bond on the basal plane. ${ }^{78,80,196,260,372-374}$ In the first report by Voiry et al., ${ }^{80} \mathrm{CE}-1 \mathrm{~T}-\mathrm{MoS}_{2},-\mathrm{WS}_{2}$ and $-\mathrm{MoSe}_{2}$ was reacted with alkyl halides (or an aryl diazonium salt). Similar to the first covalent functionalization of graphene, ${ }^{375}$ the electrophile attacks the negatively charged nanosheets. On charge neutralization, a covalent carbon-chalcogen bond is formed on the basal plane with degrees of functionalization of $20 \%$ (with respect to functional groups per $\mathrm{MX}_{2}$ unit). The successful functionalization was evidenced by ${ }^{13} \mathrm{C}-\mathrm{NMR}$ spectroscopy, IR spectroscopy and X-ray photoemission spectroscopy (XPS). XPS spectra of functionalized 1T-MoS2 (Fig. 16a) show evidence for a S-C in the S core level spectra in addition to the characteristic features of $1 \mathrm{~T}$, and $2 \mathrm{H}-\mathrm{MoS}_{2}$. The chalcogen-carbon bond is further observed in the IR spectra (Fig. 16b) in addition to the alkyl vibrations of the functional group. Importantly, the peak frequency shifts depending on the chalcogen as would be expected. The 1T-polytype is retained, but exhibits semiconducting properties after covalent functionalization due to the charge neutralization.

In a subsequent work, the question was addressed whether defects often present on CE-1T$\mathrm{MX}_{2}$ are required for successful functionalization. ${ }^{260}$ This was achieved by intercalating $\mathrm{MoS}_{2}$ with excess $\mathrm{MoS}_{2}$ over $n$-BuLi. Compared to the traditional intercalation that uses excess $n$ BuLi, a significant portion of the $\mathrm{MX}_{2}(\sim 50 \%)$ remains in its $2 \mathrm{H}$-polytype as confirmed by XPS (Fig. 16c, d) and thermogravimetric analysis (TGA) revealed that the nanosheets are less 
defective (Fig. 16e). Covalent functionalization of $\mathrm{CE}-2 \mathrm{H} / 1 \mathrm{~T}-\mathrm{MoS}_{2}$ with an aryldiazonium salt was evidenced (by XPS, IR spectroscopy and TGA coupled to mass spectrometry), albeit with lower degrees of functionalization ( $\sim 10 \%$ per $\mathrm{MoS}_{2}$ unit). In spite of this lower degree of functionalization, it is clear that defects or the 1T-polytype are not a prerequisite for the reductive functionalization and it will be possible to tailor the degree of surface decoration via the amount of charges initially present on the CE-MX $\mathrm{M}_{2}$ The 1T-polytype was found to convert rapidly (within a few days) into the $2 \mathrm{H}$-polytype in the case of the $\mathrm{CE}-2 \mathrm{H} / 1 \mathrm{~T}-\mathrm{MoS}_{2}$ from intercalation using excess $\mathrm{MoS}_{2}$. However, it is stabilized after functionalization. In analogy to the work by Voiry et al., ${ }^{80}$ it was suggested that it exhibits semiconducting properties. This covalent functionalization has subsequently been used to anchor various organic functionalities, ${ }^{78,372-374}$ or to graft molecules on the TMD surface the can be used as ligands for further derivatization via coordination chemistry. ${ }^{373,374}$ This approach is particularly interesting for catalytic applications. In a recent study, it has also been shown that 4-carboxy benzene diazonium salt can be reacted with edges of $\mathrm{LPE}-2 \mathrm{H}-\mathrm{MoS}_{2}$ in the presence of an amine catalyst. ${ }^{196}$ Cross-linking of the edges in a network via hydrogen bonding provided the proof of concept that such edge functionalization sequences can play an important role in (opto)electronics in the future, as the authors observe a drastically increased charge-carrier mobility in the network. It is worth noting that despite $\mathrm{MoS}_{2}$ nanosheets are known to behave as unipolar $n$-type semiconductors, the authors reported an unusual unipolar $p$-type transport, whose origin cannot be easily rationalized.

Alternatively, the basal plane of 2H-LPE-TMDs can be decorated by cationic metal complexes using the TMD chalcogens as ligands in a Lewis acid-base type of coordination chemistry. ${ }^{110,376}$ This strategy builds on the work of Tremel et al on $\mathrm{MoS}_{2}$-fullerenes ${ }^{377}$ and has recently been adapted for the functionalization of $\mathrm{InSe} .{ }^{378} \mathrm{In}$ particular $\mathrm{Cu}^{2+}$ ions were identified as suitable anchors to the TMD chalcogens (as evidenced by IR spectroscopy and 
XPS). This strategy is interesting, as it is a rare example of the chemical basal plane functionalization of the semiconducting 2H-polytype which is retained after the functionalization. ${ }^{110}$ However, it remains little explored and further work will be required to demonstrate its versatility as well as possibilities of further derivatization.

The most commonly used chemisorption strategy exploits chalcogen vacancy defects to coordinate nucleophiles in dative bonds, in particular thiols ${ }^{112,259,379-388}$ with dithiolane ${ }^{389}$ used to a lesser extent. This functionalization concept often referred to as ligand conjugation was first introduced by Dravid and coworkers in $2013,{ }^{259}$ where CE-1T-MoS 2 was reacted with a number of thiol-terminated polyethylene glycol derivatives bearing different ionic and non-ionic headgroups. This leads to a tuneable and $\mathrm{pH}$-stable colloidal stability (Fig. 16g top). Zeta potential measurements suggest the successful decoration of the nanosheet surface, as the zeta potential could be tuned from $\sim-60-40 \mathrm{mV}$ depending on the functional group. The disappearance of SH vibrations in the IR spectra indicate that thiols are deprotonated and the S bound to the $\mathrm{MoS}_{2}$. In this strategy, CE-1T-MoS 2 is typically used, ${ }^{259,380,382-386,388,389}$ as it exhibits a higher density of sulfur vacancies than LPE-2H-MX 2 unless they are introduced on purpose. ${ }^{381}$ However, as discussed in Sections 1.1 and 4.1, such defect sites (amongst others) are also observed in micromechanically-cleaved or CVD-grown monolayers, ${ }^{69,157,158,164}$ so that this strategy is not restricted to liquid-suspended nanosheets. ${ }^{88,293,390}$ In the case of LPE$2 \mathrm{H}-\mathrm{MX}_{2}$, edge functionalization is believed to be more favourable, for example by coordinating polyacrylic acid ${ }^{192}$ or dithiolane derivatives ${ }^{194,} 195$ to metal-rich edges and thionine $^{193}$ or polybutadiene ${ }^{391}$ to sulfur-rich edges, respectively. Note that in the latter two cases, ${ }^{193,391}$ the ligand serves as an electron acceptor in the coordinative bond.

In some cases, thiol coordination to the basal plane of $\mathrm{LPE}-2 \mathrm{H}-\mathrm{MoS}_{2}$ has been suggested, ${ }^{379}$, ${ }^{387}$ but no unambiguous proof (for example by the emergence of new species in the sulfur core level spectra in XPS) has been provided. Frequently, the disappearance of the S-H vibrations 
in IR spectroscopy (for example Fig. 16g) serves as indicator for the successful defect functionalization. However, as demonstrated by McDonald and coworkers, ${ }^{261}$ this can be due to a TMD-mediated dimerization of thiols to disulfides. In their work, the authors ${ }^{261}$ added cysteine to LPE-2H-MoS 2 in isopropanol and observed a dimerization to cysteine and subsequent physisorption rather than a coordination of the thiol to the $\mathrm{MoS}_{2}$. This was a puzzling observation, in particular because no thiol coordination could be evidenced in the reference experiment using CE-1T-MoS 2 . DFT calculations by Li et al. ${ }^{183}$ suggest that such a SV-mediated dimerization can be a thermodynamically favourable process. In their reaction pathway, a $\mathrm{MoS}_{2} \mathrm{SV}$-mediated homolytic bond cleavage of the $\mathrm{S}-\mathrm{H}$ is suggested as the first step which then leads to functionalized $\mathrm{MoS}_{2}$ in an exothermic reaction prior to a potential dimerization. However, the activation energy for the dimerization was found to be high $(91.5$ $\mathrm{kJ} / \mathrm{mol}$ ) - unless free radicals are present - which does not properly account for the observation by McDonald and coworkers.

A recent theoretical work by Förster et al. ${ }^{184}$ provides an alternative explanation to the question why sometimes defect functionalization occurs and sometimes dimerization of the thiol to disulfides. Their DFT calculations revealed that, depending on the nature of defects, an addition of a thiol results in either defect healing, coordination of the sulfur bearing ligand, or formation of disulfides/hydrodisulfides. For example, if sulfur vacancies are present, the thiol can coordinate to the exposed Mo via a transition state in an endothermic reaction, where the $\mathrm{S}-\mathrm{H}$ bond is broken and the sulfur terminated molecule adsorbs to the exposed molybdenum and the hydrogen binds to a neighbouring sulfur atom on the $\mathrm{MoS}_{2}$. This is followed by either vacancy healing or chemisorption of the functional group via the sulfur (accompanied with the release of hydrogen) in exothermic reactions. Based on the energetics, the vacancy healing should be favourable. However, if sulfur adatoms are present, the formation of a disulfide can occur in an exothermic reaction via a hydrodisulfide (net energy 
release of ca. $-1 \mathrm{eV}$ ) resulting in the formation of a disulfide and structurally perfect $\mathrm{MoS}_{2}$ (adatom healing) accompanied by the release of $\mathrm{H}_{2} \mathrm{~S}$, albeit with a high activation energy of $\sim 3 \mathrm{eV}$. Energetics are more favourable if the hydrodisulfide interacts with a nearby sulfur vacancy. This theoretical work is an excellent foundation to explain the different experimental observations. For example, the work by Jeong et al. ${ }^{194}$ showed via XPS that lipoic acid coordinates to LPE-2H-MoS 2 via the dithiolane group. This is in contrast to the above mentioned work by McDonald and coworkers, where no coordination (of neither disulfide nor thiol) was observed. ${ }^{261}$ In both cases, LPE-2H-MoS 2 was used. However, the protocol differed in a significant aspect: in the case of the work by Jeong et al., ${ }^{194}$ the reagent lipoic acid was used directly as surfactant and was thus present during the LPE by sonication, while in the case of the study by McDonald and coworkers, ${ }^{261}$ the reagent cysteine was added after the exfoliation in IPA was performed. Since theoretical work has shown that oxidation of $\mathrm{MoS}_{2}$ can readily occur on both the sulfur vacant basal plane, ${ }^{392}$ as well as edge, ${ }^{393}$ the coordination of the sulfur species can potentially be prevented in ambient conditions, if oxidation occurs prior to functionalization. Furthermore, it should be noted that chalcogen and vacancy defects are not the only defects that can be present. For example, numerous antisite defects have also been observed experimentally. ${ }^{164}$ To fully exploit defect functionalization in TMDs, it will therefore be crucial to control both type and content of defects and systematically study their impact on the respective functionalization.

The chemistry of liquid-suspended TMDs can furthermore be exploited to decorate the nanosheets with noble metal (Au, Pt, Ag, Pd) nanoparticles (NPs). These structures are particularly interesting for catalysis, plasmonics or sensing. ${ }^{337,394-408}$ The growth of can be

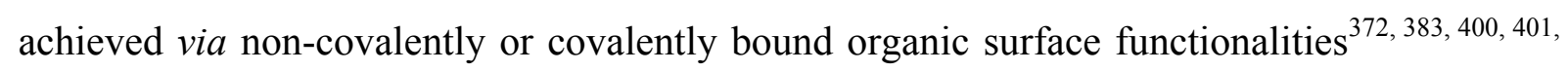
403 nicely illustrating that TMD functionalization can be used to create hybrid structures. Alternatively, NPs can be grown directly on both CE-1T-MX $\mathrm{M}_{2}$ and LPE-2H-MX ${ }_{2}$ nanosheets 
after reduction of noble metal precursor salts. ${ }^{339,} 394-399,404,409,410$ Typically, an additional reducing agent is added, ${ }^{394,396-399,409}$ even though the spontaneous formation (i.e. without additional reducing agent) has also been observed in some cases. ${ }^{339,394,395,404,410}$ This is documented to be the case when CE-1T-MoS $2{ }^{394,395,410}$ or CE-1T-WS ${ }_{2}{ }^{395}$ are used. In such a way, a number of noble metal nanoparticles like Pd, Pt or Au can be epitaxially grown on the 1T-MX 2 surface. Typical TEM images are shown in Fig. 17a-c. Notably, decoration occurs on the basal plane and XPS (for Au-decorated nanosheets in Fig. 17d) does not show evidence for a covalent binding. This is likely because the excess charges of the CE-MX $\mathrm{X}_{2}$ can reduce the metal precursor salt resulting in the nucleation of physisorbed nanoparticles on both basal plane and edge. (Note that the non-covalent binding has not been unambiguously confirmed in all reports).

In contrast to these reports on CE-1T-MoX 2 , a covalent bond formation between the sulfur of the TMD and AuNPs was demonstrated according to XPS (Fig. 17e) in hybrid materials obtained after reaction of $\mathrm{HAuCl}_{4}$ with $\mathrm{LPE}-2 \mathrm{H}-\mathrm{WS}_{2}$ in the absence of additional reducing agents. ${ }^{404}$ In this case, the NPs are predominantly located at nanosheet edges and terraces of incompletely exfoliated nanosheets (Fig. 17f). It was suggested that thiol functionalities present on edges and defect sites act as reducing agent for the initial nucleation resulting in a conversion to disulfides, as indirectly confirmed by an increased electrocatalytic activity in the hydrogen evolution reaction, where disulfides are the predominant active sites. ${ }^{411}$ Due to the higher mass of Au-decorated $\mathrm{WS}_{2}$ opposed to the native $\mathrm{WS}_{2}$ in combination with the edge decoration, an enrichment of monolayers was readily achieved by centrifugation resulting in $\mathrm{WS}_{2}$ dispersions with monolayer volume fractions of 50-90\% (depending on the initial lateral size of the LPE-WS 2 ) with a sparse decoration of nanoparticles around the edges (Fig. 17g). The optical properties of the semiconducting 2H-polytype (e.g. photoluminescence 
of monolayers) were retained making these hybrids potentially interesting for printed optoelectronics.

By and large, the (multi)functional inks developed by combining TMDs with suitably designed molecules can express unique functionalities which are resulting from those determined by the individual components. Their processing and integration for device applications (e.g. in opto-electronics, photonics, sensing, etc.) can be carried out using upscalable methods such as printing (ink-jet, roll-to-roll, etc.) and spray-coating, and the devices can be easily supported on flexible foils, opening new intriguing perspectives in the fields of flexible, foldable and wearable (opto)electronics. 
Table 2. Functionalization of solution-processed group-6 TMD nanosheets via physisorption of molecules, polymers and biopolymers.

\begin{tabular}{|c|c|c|c|c|c|c|}
\hline Ref. & Interaction & Molecule or (bio)polymers & 2D material & $\begin{array}{l}\text { Exfoliation } \\
\text { Method }\end{array}$ & Medium & Reason for functionalization \\
\hline & & Molecules & & & & \\
\hline 319 & van der Waals & sodium cholate (SC) & $2 \mathrm{H}-\mathrm{MoS}_{2}$ & sonication & aqueous & stabilization in LPE \\
\hline 322 & van der Waals & $p$-phosphonic acid calix[8]arene & $2 \mathrm{H}-\mathrm{MoS}_{2}, 2 \mathrm{H}-\mathrm{WS}_{2}$ & sonication & aqueous & stabilization in LPE \\
\hline 323 & van der Waals & sodium dodecyl sulfate (SDS) & $2 \mathrm{H}-\mathrm{MoS}_{2}$ & ball milling & aqueous & stabilization in LPE, gas sensing \\
\hline 369 & electrostatic & $\begin{array}{l}\text { hexadecyltrimethylammonium } \\
\text { chloride (CTAC) }\end{array}$ & $1 \mathrm{~T}-\mathrm{MoS}_{2}$ & $\mathrm{CE} n$-BuLi & aqueous & stabilization after chemical exfoliation \\
\hline 325 & van der Waals & alkyl-trichlorosilanes & $2 \mathrm{H}-\mathrm{MoS}_{2}$ & sonication & $\begin{array}{l}1,2 \text { dichloro- } \\
\text { benzene }\end{array}$ & stabilization after LPE, thin film formation \\
\hline 370 & electrostatic & $\begin{array}{l}\text { cetyltrimethylammonium bromide (CTAB), } \\
\text { chitosan (CS) }\end{array}$ & $1 \mathrm{~T}-\mathrm{MoS}_{2}$ & $\begin{array}{l}\mathrm{CE} n \text {-BuLi } \\
\text { (hydrothermal) }\end{array}$ & $\begin{array}{l}\text { aqueous, then } \\
\text { redispersion in } \\
\text { dimethylformamide }\end{array}$ & $\begin{array}{l}\text { transfer to organic solvent; polymer } \\
\text { composites }\end{array}$ \\
\hline 324 & van der Waals & sodium cholate (SC) & $2 \mathrm{H}-\mathrm{MoS}_{2}, 2 \mathrm{H}-\mathrm{WS}_{2}$ & sonication & aqueous & stabilization in LPE for size selection \\
\hline 371 & electrostatic & oleylamine & $1 \mathrm{~T}-\mathrm{MoS}_{2}$ & CE $n$-BuLi & $\begin{array}{l}\text { aqueous, then } \\
\text { transfer to various } \\
\text { organic }\end{array}$ & $\begin{array}{l}\text { phase transfer to organic solvents; } \\
\text { conversion to } 2 \mathrm{H}-\mathrm{MoS}_{2} \text { in high boiling point } \\
\text { solvent }\end{array}$ \\
\hline 326 & van der Waals & $\begin{array}{l}\text { sodium dodecyl sulfate (SDS), } \\
\text { cetyltrimethylammonium bromide (CTAB) }\end{array}$ & $2 \mathrm{H}-\mathrm{MoS}_{2}$ & sonication & aqueous & stabilization in LPE \\
\hline 105 & van der Waals & sodium cholate (SC) & $2 \mathrm{H}-\mathrm{MoS}_{2}$ & shear exfoliation & aqueous & stabilization in LPE \\
\hline 327 & van der Waals & sodium deoxycholate (SDC) & $2 \mathrm{H}-\mathrm{MoS}_{2}$ & sonication & aqueous & $\begin{array}{l}\text { stabilization in LPE, mixing with PVA for } \\
\text { composite fabrication }\end{array}$ \\
\hline 328 & van der Waals & sodium cholate (SC) & $2 \mathrm{H}-\mathrm{WS}_{2}$ & sonication & aqueous & stabilization in LPE for size selection \\
\hline 329 & van der Waals & sodium cholate (SC) & $2 \mathrm{H}-\mathrm{MoS}_{2}, 2 \mathrm{H}-\mathrm{WS}_{2}$ & dry ball milling & aqueous & stabilization \\
\hline 330 & van der Waals & $\begin{array}{l}\text { 2,3,6,7,10,11-hexahydroxytriphenylene and } \\
\text { derivatives }\end{array}$ & $2 \mathrm{H}-\mathrm{MoS}_{2}$ & $\begin{array}{l}\text { sonication and dry } \\
\text { ball milling }\end{array}$ & various & stabilization in LPE \\
\hline
\end{tabular}




\section{Polymers or biopolymers}

polyoxyethylene sorbitan monooleate (Tween 80 ), polyoxyethylene sorbitan trioleate (Tween 85),

polyvinylpyrrolidone (PVP), polyoxyethylene

ther (Brij 700), polyoxyethylene octyl phenyl

ether (Triton X-100), gum arabic, Pluronic P-123,

n-Dodecyl $\beta$-D-maltoside (DBDM)

polybutadiene (PBD)

poly(styrene-co-butadiene) (PBS)

polystyrene (PS),

poly(vinyl chloride) (PVC)

334 van der Waals

poly(vinyl acetate) (PVAc)

polycarbonate (PC),

poly(methyl methacrylate) (PMMA),

poly(vinylidene chloride) (PVDC),

cellulose acetate (CA)

van der Waals

gelatine

van der Waals

Pluronic F108

\section{Pluronic P123, Pluronic F127}

pyrene-derivatized hyaluronic acid

electrostatic

assembly on poly(allylamine hydrochloride)

(PAH), and poly(styrenesulfonate) (PSS)

van der Waals

Pluronic F68

van der Waals

polyvinylpyrrolidone (PVP)

van der Waals

chitosan
$2 \mathrm{H}-\mathrm{MoS}_{2}, 2 \mathrm{H}-\mathrm{WS}_{2}$

sonication

aqueous

stabilization in LPE sonication

-MoS

$2 \mathrm{H}-\mathrm{MoS}_{2}, 2 \mathrm{H}-\mathrm{WS}$

$2 \mathrm{H}-\mathrm{WS}_{2}$

$2 \mathrm{H}-\mathrm{MoS}_{2}$

$2 \mathrm{H}-\mathrm{MoS}_{2}$

$1 \mathrm{~T}-\mathrm{MoS}_{2}$

$2 \mathrm{H}-\mathrm{MoS}_{2}, 2 \mathrm{H}-\mathrm{WS}$

$2 \mathrm{H}-\mathrm{MoS}_{2}$

$2 \mathrm{H}-\mathrm{MoS}_{2}$

$2 \mathrm{H}-\mathrm{MoS}_{2}$, partial conversion to $1 \mathrm{~T}$ sonication

sonication

sonication

sonication

CE $n$-BuLi

sonication

sonication and supercritical $\mathrm{CO}_{2}$

sonication

sonication aqueous

aqueous

aqueous

aqueous

aqueous

aqueous

ethanol $/ \mathrm{H}_{2} \mathrm{O}$

aqueous

methanol, then redispersion in $\mathrm{H}_{2} \mathrm{O}$ stabilization in LPE

stabilization in LPE, gelatine composites

stabilization in LPE

stabilization in LPE, photocatalysis

stabilization in LPE

electrostatic layer by layer assembly

thickness sorting in density gradient after LPE

stabilization in LPE, cell labelling

stabilization in LPE, chitosan composites

Stabilization in LPE, farbication of $\mathrm{H}_{2} \mathrm{O}_{2}$ sensor 


\begin{tabular}{|c|c|c|}
\hline 350 & van der Waals & chitosan \\
\hline 351 & van der Waals & bovine serum albumin, then methylene blue \\
\hline 338 & van der Waals & polyvinylpyrrolidone (PVP) \\
\hline 339 & van der Waals & Pluronic P123 \\
\hline 340 & van der Waals & poly(ureamethylvinyl)silazane \\
\hline 401 & van der Waals & polydopamine \\
\hline 352 & van der Waals & bovine serum albumin \\
\hline 353 & van der Waals & DNA oligonucleotides \\
\hline 354 & van der Waals & DNA oligonucleotides \\
\hline 355 & van der Waals & nanofibrillated cellulose \\
\hline 356 & van der Waals & alkali lignin \\
\hline 357 & van der Waals & guar gum, xanthan gum, tannic acid \\
\hline 358 & van der Waals & chitosan \\
\hline 359 & van der Waals & chitosan \\
\hline 341 & van der Waals & $\begin{array}{l}19 \text { different poloxamers (i.e., Pluronics and } \\
\text { Tetronics) }\end{array}$ \\
\hline 343 & van der Waals & polyvinylalcohol (PVA) \\
\hline 344 & van der Waals & Pluronic F108 \\
\hline 342 & van der Waals & $\begin{array}{l}\text { tetrathiafulvalene (TTF)- } \\
\text { substituted polymers }\end{array}$ \\
\hline
\end{tabular}

\begin{tabular}{|c|c|c|c|}
\hline $2 \mathrm{H}-\mathrm{MoS}_{2}$ & $\begin{array}{l}\text { sonication after } \\
\text { oleum pre- } \\
\text { treatment }\end{array}$ & aqueous & $\begin{array}{l}\text { stabilization, chemotherapeutic drug } \\
\text { nanocarrier }\end{array}$ \\
\hline Acid-exfoliated $\mathrm{WS}_{2}$ & $\begin{array}{l}\mathrm{H}_{2} \mathrm{SO}_{4} \\
\text { intercalation }\end{array}$ & aqueous & stabilization, photosensitization \\
\hline $2 \mathrm{H}-\mathrm{MoS}_{2}$ & sonication & various & stabilization in LPE, film formation \\
\hline $2 \mathrm{H}-\mathrm{MoS}_{2}$ & sonication & aqueous & $\begin{array}{l}\text { stabilization in LPE, anchor for AuNPs to } \\
\text { enhance photocurrent }\end{array}$ \\
\hline $\begin{array}{l}\text { Acid exfoliated } \\
\mathrm{MoS}_{2}\end{array}$ & $\begin{array}{l}\text { chlorosulfonic } \\
\text { acid exfoliation }\end{array}$ & aqueous & $\begin{array}{l}\text { formation of amorphous } \mathrm{SiCN} \text { ceramic for } \\
\mathrm{Li} \text { ion batteries }\end{array}$ \\
\hline $1 \mathrm{~T}-\mathrm{MoS}_{2}$ & $\mathrm{CE} n$-BuLi & aqueous & $\begin{array}{l}\text { stabilization after chemical exfoliation, } \\
\text { nanoparticle decoration }\end{array}$ \\
\hline $2 \mathrm{H}-\mathrm{MoS}_{2}$ & sonication & aqueous & $\begin{array}{l}\text { stabilization in LPE, biocompatible } \\
\text { nanosheets }\end{array}$ \\
\hline $1 \mathrm{~T}-\mathrm{MoS}_{2}$ & $\mathrm{CE} n$-BuLi & aqueous & $\begin{array}{l}\text { surface functionalization for a prostate } \\
\text { specific antigen sensor }\end{array}$ \\
\hline $2 \mathrm{H}-\mathrm{MoS}_{2}$ & $\begin{array}{l}\text { sonication } \\
\text { (initially in SC) }\end{array}$ & aqueous & stabilization at high ionic strength \\
\hline $2 \mathrm{H}-\mathrm{MoS}_{2}$ & sonication & aqueous & stabilization in LPE, composite formation \\
\hline $2 \mathrm{H}-\mathrm{MoS}_{2}, 2 \mathrm{H}-\mathrm{WS}_{2}$ & sonication & aqueous & stabilization in LPE \\
\hline $2 \mathrm{H}-\mathrm{MoS}_{2}$ & sonication & aqueous & stabilization in LPE \\
\hline $2 \mathrm{H}-\mathrm{MoS}_{2}$ & sonication & $\begin{array}{l}\text { aqueous, then } \\
\text { tetrahydrofuran }\end{array}$ & $\begin{array}{l}\text { stabilization in LPE, composite formation } \\
\text { (epoxy resin) in tetrahydrofuran }\end{array}$ \\
\hline $2 \mathrm{H}-\mathrm{MoS}_{2}$ & $\begin{array}{l}\text { ionic liquid (IL) } \\
\text { assisted grinding }\end{array}$ & IL, then aqueous & biocompatible nanosheets in water \\
\hline $2 \mathrm{H}-\mathrm{MoS}_{2}$ & sonication & aqueous & $\begin{array}{l}\text { stabilization in LPE, biocompatible } \\
\text { dispersions }\end{array}$ \\
\hline $2 \mathrm{H}-\mathrm{WS}_{2}$ & sonication & aqueous & stabilization in LPE, composite fabrication \\
\hline $2 \mathrm{H}-\mathrm{WS}_{2}$ & sonication & aqueous & $\begin{array}{l}\text { stabilization in LPE, isolation of nanodots } \\
\text { for HER }\end{array}$ \\
\hline $1 \mathrm{~T}-\mathrm{MoS}_{2}$ & $\mathrm{CE} n$-BuLi & $\begin{array}{l}\text { redispersion in } \\
\text { tetrahydrofuran }\end{array}$ & $\begin{array}{l}\text { stabilization after chemical exfoliation, } \\
\text { chemical doping }\end{array}$ \\
\hline
\end{tabular}


$2 \mathrm{H}-\mathrm{WS}_{2}, 2 \mathrm{H}-\mathrm{WSe}_{2}$

sonication

$2 \mathrm{H}-\mathrm{MoS}_{2}$

sonication

cross-beta-amyloid (protein nanotubes)

van der Waals

stabilization in LPE, biocompatible nanosheets

stabilization in LPE, biocompatible nanosheets

stabilization in LPE, stimuli responsive dispersions 
Table 3. Functionalization of solution-processed TMD nanosheets via coordination chemistry on defects (dative bonds of nucleophiles) and on basal plane (Lewis acid-base chemistry).

\begin{tabular}{|c|c|c|c|c|c|c|}
\hline Ref. & Suggested binding & Functional group & 2D material & $\begin{array}{l}\text { Exfoliation } \\
\text { Method }\end{array}$ & Medium & Reason for functionalization \\
\hline & \multicolumn{6}{|c|}{ Coordination chemistry on defects (dative bonds of nucleophiles) } \\
\hline 259 & $\begin{array}{l}\text { thiol coordination to } S \\
\text { vacancies }\end{array}$ & $\begin{array}{l}\text { thiol-terminated polyethylene glycol with } \\
\text { various headgroups }\end{array}$ & $1 \mathrm{~T}-\mathrm{MoS}_{2}$ & $\mathrm{CE} n$-BuLi & aqueous & proof of concept \\
\hline 379 & $\begin{array}{l}\text { thiol coordination to } S \\
\text { vacancies + van der Waals }\end{array}$ & thioglycolic acid & $2 \mathrm{H}-\mathrm{MoS}_{2}$ & sonication & aqueous & stabilization in LPE \\
\hline 389 & $\begin{array}{l}\text { dithiolane coordination to } \\
\mathrm{S} \text { vacancies }\end{array}$ & lipoic acid conjugated polyethylene glycol & $1 \mathrm{~T}-\mathrm{WS}_{2}, 1 \mathrm{~T}-\mathrm{MoS}_{2}$ & $\mathrm{CE} n$-BuLi & aqueous & $\begin{array}{l}\text { biocompatible nanosheets as theranostic } \\
\text { agent or in drug delivery }\end{array}$ \\
\hline 380 & $\begin{array}{l}\text { thiol coordination to } S \\
\text { vacancies }\end{array}$ & dithioglycol & $1 \mathrm{~T}-\mathrm{MoS}_{2}$ & $\begin{array}{l}\text { CE } n \text {-BuLi } \\
\text { (hydrothermal) }\end{array}$ & tetrahydrofuran & $\begin{array}{l}\text { nanocomposites with octa-vinyl polyhedral } \\
\text { oligomeric silsesquioxanes (improved } \\
\text { thermal, mechanical, flame-retardant } \\
\text { properties) }\end{array}$ \\
\hline 381 & $\begin{array}{l}\text { thiol coordination to } S \\
\text { vacancies }\end{array}$ & mercaptoundecanoic acid & $2 \mathrm{H}-\mathrm{MoS}_{2}$ & $\begin{array}{l}\text { sonication (to } \\
\text { introduce defects) }\end{array}$ & ethanol $/ \mathrm{H}_{2} \mathrm{O}$ & sensor for volatile organic compounds \\
\hline 382 & $\begin{array}{l}\text { thiol coordination to } S \\
\text { vacancies }\end{array}$ & dodecanethiol & $1 \mathrm{~T}-\mathrm{MoS}_{2}$ & $\mathrm{CE} n$-BuLi & acetone & proof of concept: transfer to organic solvents \\
\hline 192 & $\begin{array}{l}\text { coordination of } \mathrm{COOH} \text { to } \\
\mathrm{S} \text { vacancies } \\
\text { (predominantly. at edges) }\end{array}$ & polyacrylic acid & $2 \mathrm{H}-\mathrm{WS}_{2}$ & sonication & aqueous & $\begin{array}{l}\text { stabilization in LPE and fabrication of a } \\
\text { biosensor with adsorbed ss-DNA }\end{array}$ \\
\hline 383 & $\begin{array}{l}\text { thiol coordination to } S \\
\text { vacancies }\end{array}$ & $\begin{array}{l}\text { mercaptopropionic acid, 1-Thioglycerol, L- } \\
\text { Cysteine }\end{array}$ & $1 \mathrm{~T}-\mathrm{MoS}_{2}$ & $\begin{array}{l}\text { CE } n \text {-BuLi } \\
\text { (hydrothermal) }\end{array}$ & aqueous & $\begin{array}{l}\text { proof of concept; further derivatization by in } \\
\text { situ reduction of metal ions, esterification, } \\
\text { polymerizations }\end{array}$ \\
\hline 384 & $\begin{array}{l}\text { thiol coordination to } S \\
\text { vacancies }\end{array}$ & $\begin{array}{l}\text { meso-2,3-dimercaptosuccinnic acid- } \\
\text { modified iron oxide nanoparticles, then } \\
\text { lipoic acid terminated polyethylene glycol } \\
\text { and amine-terminated branched PEG }\end{array}$ & $1 \mathrm{~T}-\mathrm{MoS}_{2}$ & $\mathrm{CE} n$-BuLi & aqueous & $\begin{array}{l}\text { TMD-based nanoplatform for multimodal } \\
\text { imaging-guided photothermal therapy of } \\
\text { cancer }\end{array}$ \\
\hline 112 & $\begin{array}{l}\text { thiol coordination to } S \\
\text { vacancies }\end{array}$ & $\begin{array}{l}\text { p-mercaptophenol, thiophenol, } \\
\text { 1-propanethiol, 1-nonanethiol, and 1- } \\
\text { dodecanethiol }\end{array}$ & $2 \mathrm{H}-\mathrm{MoS}_{2}$ & $\begin{array}{l}\text { two solvent } \\
\text { grinding assisted } \\
\text { sonication }\end{array}$ & ethanol & $\begin{array}{l}\text { proof of concept } \\
\text { (comparison of various thiols) }\end{array}$ \\
\hline
\end{tabular}


no thiol coordination, but physisorbed dimer

dithiolane coordination to

194

vacancies mainly at

edges

thiol coordination to $\mathrm{S}$

vacancies

thiol coordination to $S$

vacancies

thiol coordination to $S$

vacancies

thiol coordination to $\mathrm{S} / \mathrm{Se}$

vacancies

sulfur addition at

molybdenum edges physisorbed cystine produced from cysteine

$2 \mathrm{H}-\mathrm{MoS}_{2}$

$2 \mathrm{H}-\mathrm{MoS}_{2}$

thiol-terminated tetra ethylene glycol with various head groups

thioglycolic acid

$\mathrm{N}, \mathrm{N}^{\prime}$-bis(p-thiophenylamido)

diethylenetriamine- $\mathrm{N}, \mathrm{N}^{\prime}, \mathrm{N}^{\prime \prime}$-triacetic and

complexation with Eu3+ and Gd3+

\section{thiobarbituric acid}

1,2-dithiolane derivatives bearing ethylene

glycol alkyl chain terminated to a

butoxycarbonyl (BOC)-protected amine or a

pyren

$2 \mathrm{H}-\mathrm{MoS}_{2}$

$2 \mathrm{H}-\mathrm{MoS}_{2}$ sonication

isopropanol

proof of concept

1T-MoS, $1 \mathrm{~T}-\mathrm{WS}_{2}$,

$1 \mathrm{~T}-\mathrm{MoSe}_{2}, 1 \mathrm{~T}-\mathrm{WSe}_{2}$
CE $n$-BuLi

aqueous

$\mathrm{H}_{2} \mathrm{O}, \mathrm{H}_{2} \mathrm{O}$ /tetra-

hydrofuran for

composite

(hydrothermal)

sonication

isopropanol

CE $n$-BuLi

acid-assisted aqueous

chlorosulfonic acid

then (sonication) dimethylformamide acceptor hybrids

proof of concept

2D material antibiotic

polymer composite (chitosan, improved mechanical and thermal properties)

proof of concept, introduction of magnetic and tailored light emission

proof of concept, preservation of metallic 1T

phase

\section{Coordination chemistry on basal plane (Lewis acid-base chemistry)}

\section{$376 \quad \operatorname{Metal}\left(\mathrm{Cu}^{2+}\right)$ ion} coordination

Metal ion $\left(\mathrm{Cu}^{2+}, \mathrm{Ni}^{2+}\right.$,

$\mathrm{Zn}^{2+}$ ) coordination cross-linking at edge sites via polymer

acetates (via metal cations)
$2 \mathrm{H}-\mathrm{MoS}_{2}$

$2 \mathrm{H}-\mathrm{MoS}_{2}$ sonication

sonication mechanical robustness, polymer composite, dimethylformamide reduced graphene oxide ( $\mathrm{rGO}$ ) composite as electrode in $\mathrm{Li}$ ion batteries

proof of concept 
Table 4. Reductive covalent basal plane functionalization of solution-processed TMDs with electrophiles. Notes: $(*)$ Different intercalation conditions.

\begin{tabular}{|c|c|c|c|c|c|c|}
\hline Ref. & Reagent & Functional group & 2D-material & $\begin{array}{l}\text { Exfoliation } \\
\text { Method }\end{array}$ & Medium & Reason for functionalization \\
\hline 80 & $\begin{array}{l}\text { via alkyl halides, aryl } \\
\text { diazonium salts }\end{array}$ & alkyl, 4-methoxybenzene & $\begin{array}{l}1 \mathrm{~T}-\mathrm{MoS}_{2}, 1 \mathrm{~T}-\mathrm{WS}_{2} \\
1 \mathrm{~T}-\mathrm{MoSe}_{2}\end{array}$ & $\mathrm{CE} n$-BuLi & aqueous & proof of concept \\
\hline 260 & via aryldiazonium salts & 4-methoxybenzene & $1 \mathrm{~T} / 2 \mathrm{H}-\mathrm{MoS}_{2}$ & $\begin{array}{l}\text { CE } n \text {-BuLi } \\
(*)\end{array}$ & aqueous & proof of concept \\
\hline 196 & $\begin{array}{l}\text { via } 4 \text {-carboxy- } \\
\text { benzenediazonium salt }\end{array}$ & 4-carboxybenzene & 1T-MoS2 & $\mathrm{CE} n$-BuLi & $\begin{array}{l}\text { dimethylformamide } \\
\text { (DMF) }\end{array}$ & $\begin{array}{l}\text { reference system to edge functionalized } 2 \mathrm{H}- \\
\mathrm{MoS}_{2} \text { in thin film transistor }\end{array}$ \\
\hline 78 & via organohalides & $\begin{array}{l}\text { benzene, 4-methoxybenzene, } 4 \text { - } \\
\text { nitrobenzene, porphyin, pyrene derivatives }\end{array}$ & $\begin{array}{l}1 \mathrm{~T}-\mathrm{MoS}_{2}, 1 \mathrm{~T}- \\
\mathrm{MoSe}_{2}, 2 \mathrm{H}-\mathrm{MoS}_{2} \\
\text { and } 2 \mathrm{H} \mathrm{MoSe} \text { (with }_{2} \\
\text { Pd0 catalyst) }\end{array}$ & $\begin{array}{l}\mathrm{CE} n \text {-BuLi, } \\
\text { sonication }\end{array}$ & $\begin{array}{l}\mathrm{H}_{2} \mathrm{O} / \mathrm{DMF}(1 \mathrm{~T}) \\
\operatorname{DMF}(2 \mathrm{H})\end{array}$ & proof of concept \\
\hline 373 & $\begin{array}{l}\text { via 4-Cyanobenzyl } \\
\text { diazonium salt }\end{array}$ & $\begin{array}{l}\text { 4-cyanobenzol and coordination to } \\
\text { cobaloxime }\end{array}$ & $1 \mathrm{~T}-\mathrm{MoS}_{2}$ & $\mathrm{CE} n$-BuLi & aqueous & improved HER electrocatalyst \\
\hline 374 & $\begin{array}{l}\text { via } 5 \text {-Bromomethyl-2,2'- } \\
\text { bipyridine }\end{array}$ & $\begin{array}{l}\text { methyl-2,2'-bipyridine and coordination to } \\
\text { RuII(bpy)2Cl2 }\end{array}$ & 1T-MoS2 & $\mathrm{CE} n$-BuLi & $\mathrm{H}_{2} \mathrm{O}$ /ethanol & improved HER electrocatalyst \\
\hline
\end{tabular}


Table 5. Decoration of solution-processed TMDs with noble metal nanoparticles.

\begin{tabular}{|c|c|c|c|c|c|c|}
\hline Ref. & $\begin{array}{l}\text { Method/suggested } \\
\text { binding }\end{array}$ & Nanoparticle and 2D material & $\begin{array}{l}\text { Exfoliation } \\
\text { Method }\end{array}$ & Precursor Salt & $\begin{array}{l}\text { Reducing } \\
\text { Agent }\end{array}$ & Reason for functionalization \\
\hline 394 & $\begin{array}{l}\text { epitaxial growth on basal } \\
\text { plane }\end{array}$ & $\mathrm{Pd}, \mathrm{Pt}, \mathrm{Au}$ on $\mathrm{MoS}_{2}$ & $\begin{array}{l}\text { electrochemical } \mathrm{Li} \\
\text { intercalation }\end{array}$ & $\begin{array}{l}\mathrm{K}_{2} \mathrm{PdCl}_{4}, \mathrm{~K}_{2} \mathrm{PtCl}_{4} \\
\mathrm{HAuCl}_{4}\end{array}$ & $\begin{array}{l}\text { Pd: CTAB/ } \\
\text { ascorbic acid; Pt: } \\
\text { trisodium citrate; } \\
\text { Au: Trisodium } \\
\text { citrate }\end{array}$ & $\begin{array}{l}\text { proof of concept, hybrids for } \\
\text { electrocatalysis }\end{array}$ \\
\hline 395 & $\begin{array}{l}\text { spontaneous formation at } \\
\text { defect sites and edges } \\
\text { (physisorption) }\end{array}$ & $\mathrm{Au}$ on $1 \mathrm{~T}-\mathrm{MoS}_{2}, 1 \mathrm{~T}-\mathrm{WS}_{2}$ & $\mathrm{CE} n$-BuLi & $\mathrm{HAuCl}_{4}$ & none & $\begin{array}{l}\text { proof of concept, hybrids for } \\
\text { electrocatalysis }\end{array}$ \\
\hline 409 & $\begin{array}{l}\text { metal nuclei seeding on } \\
\text { defect sites (basal plane) }\end{array}$ & $\mathrm{Au}, \mathrm{Ag}$ on $2 \mathrm{H}-\mathrm{MoS}_{2}$ & sonication in NMP & $\mathrm{HAuCl}_{4}, \mathrm{AgNO}_{3}$ & $\begin{array}{l}\text { hydroxyl amine, } \\
\text { microwave }\end{array}$ & $\begin{array}{l}\text { proof of concept, improve transport } \\
\text { behavior }\end{array}$ \\
\hline 396 & $\begin{array}{l}\text { self-assembly on basal } \\
\text { plane }\end{array}$ & $\mathrm{Au}$ on $2 \mathrm{H}-\mathrm{MoS}_{2}$ & $\begin{array}{l}\text { L-cysteine assisted } \\
\text { exfoliation }\end{array}$ & $\mathrm{HAuCl}_{4}$ & $\mathrm{NaBH}_{4}$ & $\begin{array}{l}\text { fabrication of an aptasensor via layer by } \\
\text { layer deposition }\end{array}$ \\
\hline 397 & $\begin{array}{l}\text { self-assembly on basal } \\
\text { plane }\end{array}$ & $\mathrm{Ag}$ on $2 \mathrm{H}-\mathrm{MoS}_{2}$ & $\begin{array}{l}\text { sonication in NMP } \\
(+ \text { chitosan } \\
\text { stabilizer })\end{array}$ & $\mathrm{AgNO}_{3}$ & ascorbic acid & electrocatalysis (typrophan oxidation) \\
\hline 398 & $\begin{array}{l}\text { epitaxial growth on basal } \\
\text { plane }\end{array}$ & $\mathrm{Au}$ on $\mathrm{MoS}_{2}$ & $\begin{array}{l}\text { electrochemical } \mathrm{Li} \\
\text { intercalation }\end{array}$ & $\mathrm{HAuCl}_{4}$ & ascorbic acid & $\begin{array}{l}\text { Au NP plasmon enhanced photocatalytic } \\
\text { water-splitting }\end{array}$ \\
\hline 399 & $\begin{array}{l}\text { self-assembly on basal } \\
\text { plane (physisorption) }\end{array}$ & $\mathrm{Au}, \mathrm{Ag}, \mathrm{Pt}, \mathrm{Pd}$ on $1 \mathrm{~T}-\mathrm{MoS}_{2}$ & $\begin{array}{l}\text { CE } n \text {-BuLi } \\
\text { (+carboymethyl } \\
\text { cellulose stabilizer) }\end{array}$ & $\begin{array}{l}\mathrm{HAuCl}_{4}, \mathrm{AgNO}_{3} \\
\mathrm{H}_{2} \mathrm{PtCl}_{6}, \mathrm{PdCl}_{2}\end{array}$ & microwave & $\begin{array}{l}\text { proof of concept, } \mathrm{Pd}-\mathrm{MoS}_{2} \text { for } \\
\text { electrocatalysis (methanol oxidation) }\end{array}$ \\
\hline 383 & $\begin{array}{l}\text { growth on } \mathrm{COOH} \text { on } \\
\text { basal plane functionalized } \\
\mathrm{MoS}_{2}\end{array}$ & $\mathrm{Ag}$ on functionalized $\mathrm{MoS}_{2}$ & $\begin{array}{l}\text { thiol coordination } \\
\text { after } C E\end{array}$ & $\mathrm{AgNO}_{3}$ & $\mathrm{NaBH}_{4}$ & proof of concept \\
\hline 400 & $\begin{array}{l}\text { growth on } \mathrm{COOH} \text { on } \\
\text { basal plane functionalized } \\
\mathrm{MoS}_{2}\end{array}$ & $\begin{array}{l}\mathrm{Pt}, \mathrm{Au}, \mathrm{Fe}_{3} \mathrm{O}_{4}, \mathrm{CdS}_{2}, \mathrm{PbS} \text { on functionalized } \\
\mathrm{MoS}_{2}\end{array}$ & $\begin{array}{l}\text { thiol coordination } \\
\text { after } \mathrm{CE}\end{array}$ & $\begin{array}{l}\mathrm{K}_{2} \mathrm{PtCl}_{4}, \mathrm{HClAu}_{4} \\
\mathrm{FeCl}_{3}, \mathrm{CdCl}_{2} \\
\mathrm{~Pb}\left(\mathrm{CH}_{3} \mathrm{COO}\right)_{2}\end{array}$ & $\begin{array}{l}\mathrm{Pt}, \mathrm{Au}: \mathrm{NaBH}_{4} \\
\mathrm{Fe}_{3} \mathrm{O}_{4}: \mathrm{NaOH} \\
\mathrm{CdS}_{2} \text { and } \mathrm{PbS}: \\
\mathrm{Na}_{2} \mathrm{~S}\end{array}$ & $\begin{array}{l}\text { proof of concept, production of ternary } \\
\text { systems for photocatalysis (reduction of } 4- \\
\text { nitrophenol) }\end{array}$ \\
\hline 339 & $\begin{array}{l}\text { growth on } \mathrm{MoS}_{2} \text { edges } \\
\text { and basal plane }\end{array}$ & $\mathrm{Au}$ on $2 \mathrm{H}-\mathrm{MoS}_{2}$ & $\begin{array}{l}\text { sonication in } \\
\text { aqueous Pluronic } \\
\text { P123 }\end{array}$ & $\mathrm{HAuCl}_{4}$ & none & $\begin{array}{l}\text { cathode buffer layers in organic } \\
\text { photovoltaic devices }\end{array}$ \\
\hline 401 & $\begin{array}{l}\text { growth on poly dopamine } \\
\text { layer surrounding } \mathrm{MoS}_{2}\end{array}$ & $\begin{array}{l}\mathrm{Pt}, \mathrm{Au} \text { on } 1 \mathrm{~T}-\mathrm{MoS}_{2} \text { with self-polymerised } \\
\text { dopamine }\end{array}$ & $\begin{array}{l}\text { CE, reaction with } \\
\text { dopamine } \\
\text { hydrochloride }\end{array}$ & $\mathrm{H}_{2} \mathrm{PtCl}_{6}, \mathrm{HAuCl}_{4}$ & none & proof of concept, photocatalysis \\
\hline
\end{tabular}


growth on $\mathrm{COOH}$ on

basal plane functionalized

$\mathrm{Ag}$ on functionalized 1T-MoS

$\mathrm{CE}$ and reductive

functionalization

$\mathrm{AgNO}_{3}$

growth on PVP on basal

plane of MoS2

$\mathrm{Au}, \mathrm{Au} / \mathrm{Pt}$ on $1 \mathrm{~T}-\mathrm{MoS}_{2}$ with PVP coating

(physisorption)

spontaneous formation

binding to defect sites an

edges (physisorption)

growth on PVP on basal

plane and edge

(physisorption)

chemisorption through

reaction with thiol edge

sites (and basal plane

$\mathrm{Au}$ on $1 \mathrm{~T}-\mathrm{MoS}_{2}$

Pd on 2H-WS

$\mathrm{Au}$ on $2 \mathrm{H}-\mathrm{WS}_{2}$

\section{$\mathrm{CE} n$-BuLi}

CE $n$-BuLi

LPE in NMP,

(+ PVP, ethylene

glycol)

LPE in aqueous SC
$\mathrm{HAuCl}_{4}$, then

$\mathrm{H}_{2} \mathrm{PtCl}_{6}$

$\mathrm{HAuCl}_{4}$

$\mathrm{Pd}(\mathrm{OAc})_{2}$
$\mathrm{NaBH}_{4}$

proof of concept

none for $\mathrm{Au}$, then

CTAB/acetic acid electrocatalysis (oxidation of methanol)

for $\mathrm{Pt}$

none

further derivatization via thiol click chemistry

photocatalysis (Suzuki reactions)

roof of concept (different mechanism resulting in chemisorption), monolaye enrichment, electrocatalytic hydrogen evolution 
Table 6. Additional methods for the functionalization of solution-processed TMD nanosheets.

\begin{tabular}{|c|c|c|c|c|c|c|}
\hline Ref. & Type of reaction & Functional group & Layered material & Exfoliation Method & Medium & Reason for functionalization \\
\hline 413 & oxidation & oxides and chlorosulfonic acid & $2 \mathrm{H}-\mathrm{WS}_{2}$ & $\begin{array}{l}\text { acid intercalation and } \\
\text { oxidation }\end{array}$ & $\begin{array}{l}\text { chlorosulfonic } \\
\text { acid, then water }\end{array}$ & proof of concept, electrode in Li ion battery \\
\hline 193 & $\begin{array}{l}\text { coordination to negatively } \\
\text { charged S-edge }\end{array}$ & thionine & $2 \mathrm{H}-\mathrm{MoS}_{2}$ & $\begin{array}{l}\text { ionic liquid assisted } \\
\text { sonication }\end{array}$ & $\begin{array}{l}\text { ionic liquid (IL), } \\
\text { then DMF }\end{array}$ & $\begin{array}{l}\text { fabrication of a double-stranded DNA } \\
\text { electrochemical biosensor }\end{array}$ \\
\hline 391 & $\begin{array}{l}\text { binding of edge } \mathrm{S} \text { to } \\
\text { polybutadiene }\end{array}$ & polybutadiene & $2 \mathrm{H}-\mathrm{MoS}_{2}$ & sonication & $\begin{array}{l}\text { N-methyl-2- } \\
\text { pyrrolidone }\end{array}$ & proof of concept, polymer composite \\
\hline 196 & $\begin{array}{l}\text { reaction of 4-carboxy- } \\
\text { benzenediazonium salt } \\
\text { with S-rich edges in the } \\
\text { presence of amine }\end{array}$ & 4-carboxybenzene & $2 \mathrm{H}-\mathrm{MoS}_{2}$ & $\begin{array}{l}\text { "solvation" in } \\
\text { dimethylformamide (DMF) }\end{array}$ & DMF & $\begin{array}{l}\text { cross-linking of edges of functionalized 2H- } \\
\mathrm{MoS}_{2} \text { via hydrogen bonding in thin film } \\
\text { transistor }\end{array}$ \\
\hline
\end{tabular}




\section{Recent progress in the chemistry of TMDs "beyond MoWSeS"}

Besides MoWSeS materials, other TMDs (group-6 tellurides, groups 4, 5, 7 and 10) possessing diverse chemical and physical properties are attracting nowadays a great deal of attention. In general, the electronic structures of TMDs are strongly dependent on the $d$ electron number of the transition metal. ${ }^{115}$ TMDs with many (group 7, 10) or few (group 4) $d$ electrons are prone to form $d^{2} s p^{3}$ hybridization, which results in the 1T-phase (or distorted $\left.1 \mathrm{~T}^{\prime}\right)$ structure. ${ }^{115,414}$ In terms of electronic transport, they mostly exhibit semiconducting properties. In contrast, TMDs of the group 5 and 6 are prone to form $d^{4} s p$ hybridization with $1 \mathrm{H}$ structure and behave as narrow-band metals or semimetals with superconducting properties. ${ }^{115,410}$ By taking advantage of chemical functionalization strategies, diverse and novel properties of TMDs may emerge and be used for device applications.

The interaction between ferromagnetism (FM) and superconductivity (SC) is a cutting-edge research topic. ${ }^{415}$ It is well known that FM can destroy singlet correlations responsible for SC, and therefore it is quite rare to find materials that simultaneously exhibit both properties. ${ }^{416}$ Recently, the coexistence of FM and SC was observed in chemically-exfoliated $2 \mathrm{H}-\mathrm{NbSe}_{2}$ (group 5) nanosheets upon chemical treatment with hydrazine molecules. ${ }^{114}$ Pristine $\mathrm{NbSe}_{2}$ is a superconducting yet non-magnetic material since the magnetic moment of $\mathrm{Nb}^{4+}\left(4 d^{1}\right.$ configuration) is quenched due to the $\mathrm{Nb}-\mathrm{Se}$ hybridization. Zhu et al. ${ }^{114}$ found that the adsorption of reducing hydrazine molecules on the surface of $\mathrm{NbSe}_{2}$ (Fig. 18a) induces a structural distortion, i.e. elongated $\mathrm{Nb}-\mathrm{Se}$ bonds and weaker $\mathrm{Nb}-\mathrm{Se}$ interactions. Such a distortion leads to the emergence of a net magnetic moment with ordered spin behaviour resulting in ferromagnetic properties. Fig. $18 \mathrm{~b}$ displays the magnetic hysteresis loop at $30 \mathrm{~K}$, which provides evidence for the existence of $\mathrm{FM}$ in $\mathrm{NbSe}_{2} .{ }^{114}$ Moreover, the abrupt drop in electrical resistivity $R$ occurring at temperature $T \leq 6 \mathrm{~K}$ (see Fig. 18c) confirms that the hydrazine treatment does not suppress the SC properties. Hence, the adsorption of hydrazine 
molecules is an effective method to modulate the electrical - see also discussion in Section 2.1 - and magnetic properties of TMDs via chemical approaches.

Monolayer $\mathrm{MoTe}_{2}$ (group 6) has emerged as a promising phase-change material due to the small energy difference $(\sim 31 \mathrm{meV})^{417}$ between the semiconducting $1 \mathrm{H}$ and metallic $1 \mathrm{~T}^{\prime}$ polytypes, ${ }^{146,147}$ which makes it possible to trigger phase transitions via electrostatic doping. ${ }^{147}$ Theoretical calculations by Zhou et al. ${ }^{417}$ predict that molecules $\left(\right.$ e.g. $\mathrm{H}_{2}, \mathrm{H}_{2} \mathrm{O}, \mathrm{NH}_{3}, \mathrm{CO}$ and $\mathrm{N}_{2}$ ) and atoms (e.g. Li, Na, K, $\mathrm{O}$ and $\mathrm{Cl}$ ) adsorbed on the surface of the 2D sheet can stabilize the $1 \mathrm{H}$ and $1 \mathrm{~T}^{\prime}$ phase, respectively. In this framework, new experimental studies are foreseen to explore the use of molecular approaches - for instance based on the "decoration" of the top and bottom surface (see Section 2.2) - to control the phase state and therefore also the electrical properties of ultrathin $\mathrm{MoTe}_{2}$.

Group-10 TMDs are gathering attention due to their thickness-dependent bandgap and high charge-carrier mobility. ${ }^{144,414}$ For instance, bulk $\mathrm{PdSe} \mathrm{C}_{2}$ and $\mathrm{PtSe}_{2}$ are semimetals whereas thin sheets of these materials (thickness $\leq 10 \mathrm{~nm}$ ) display small semiconducting bandgaps. ${ }^{144,411}$ FETs based on $\sim 9 \mathrm{~nm}$ thick sheets of $\mathrm{PdSe}_{2}$ (group-10) show ambipolar transport characteristics with electron/hole field-effect mobility $\left(\mu_{\mathrm{FE}}\right)$ up to $\sim 54 / 14 \mathrm{~cm}^{2} \mathrm{~V}^{-1} \mathrm{~s}^{-1}$ and $\mathrm{I}_{\mathrm{on}} / \mathrm{I}_{\mathrm{off}}$ ratio of $\sim 100 .{ }^{113}$ After a thermal annealing step under vacuum, which is commonly performed to remove environmental adsorbates (i.e. $\mathrm{H}_{2} \mathrm{O}$ and $\mathrm{O}_{2}$ ), the devices show unipolar $n$-type transport with $\mu_{\mathrm{FE}} \approx 216 \mathrm{~cm}^{2} \mathrm{~V}^{-1} \mathrm{~s}^{-1}$ and $I_{\mathrm{on}} / \mathrm{I}_{\mathrm{off}} \approx 10^{3}$ (Fig. 18d). However, ambipolar as well as unipolar $p$-type transport has also been achieved via physisorption of acceptor molecules, namely $\mathrm{F}_{4}$-TCNQ (see Section 2.1). Electrical transport measurements in Fig. 18e demonstrate that the charge-carrier density in $\mathrm{F}_{4}$-TCNQ-treated $\mathrm{PdSe}_{2}$ FETs can be effectively modulated by controlling the amount of physisorbed molecules. By increasing the number of molecular dopants, the authors succeeded in achieving a unipolar $p$-type transport (red curve in Fig. 18e). Overall, they showed that the combination of thermal annealing and molecular 
physisorption doping is a promising route to control the charge-carrier polarity in narrowbandgap semiconductors towards complementary logic devices and circuits.

Wan et $a l .{ }^{418}$ prepared and characterized a promising $n$-type flexible thermoelectric material based on hybrid superlattices of semiconducting $1 \mathrm{~T}-\mathrm{TiS}_{2}$ (group 4) and organic cations. $\mathrm{TiS}_{2}$ single crystals were electrochemically intercalated with hexylammonium (HA) ions dissolved in dimethyl sulfoxide (DMSO). After solvent exchange from DMSO to water the resulting compound - i.e. $\mathrm{TiS}_{2}\left[(\mathrm{HA})_{0.08}\left(\mathrm{H}_{2} \mathrm{O}\right)_{0.22}(\mathrm{DMSO})_{0.03}\right]$ - showed a remarkably low in-plane thermal conductivity $\left(\sim 0.12 \mathrm{Wm}^{-1} \mathrm{~K}^{-1}\right)$, significantly lower than that of pristine $\mathrm{TiS}_{2}$ crystals $\left(4.2 \mathrm{Wm}^{-1} \mathrm{~K}^{-1}\right)$ where the layers interact via weak van der Waals forces. On the other hand, negatively-charged $\mathrm{TiS}_{2}$ layers and organic cations in the hybrid system are coupled via strong electrostatic interactions, ${ }^{418}$ which degrade the in-plane thermal conductivity due to phonon scattering. The combination of high electrical conductivity - typical of $\mathrm{TiS}_{2}-$ with the low thermal conductivity of the intercalated compound, allowed achieving a figure of merit $Z T$ as high as $\sim 0.28(T \approx 373 \mathrm{~K})$, which is close to that of the most promising $p$-type organic thermoelectric materials, i.e. poly(3,4-ethylenedioxythiophene) polystyrene sulfonate (PEDOT:PSS).

Finally, ultrathin $\operatorname{ReSe}_{2}$ (group 7) is an appealing material for optoelectronic applications due to its wide photoresponse range. The performance of photodetectors based on $\mathrm{ReSe}_{2}$ nanosheets has been significantly improved by $n$-type doping via physisorption of triphenylphosphine $\left(\mathrm{PPh}_{3}\right)$ molecules. ${ }^{101}$ In comparison to their untreated counterparts, $\mathrm{PPh}_{3}$ treated $\mathrm{ReSe}_{2}$ FETs were found to display lower contact resistance, higher charge-carrier mobility, as well as enhanced photocurrent - by approximately a factor of 4 - under monochromatic light irradiation $(\lambda \approx 520 \mathrm{~nm})$.

By taking advantage of the exotic properties of TMDs beyond group 6 and of their tunability via molecular chemistry methods, advanced optoelectronic, magnetic, as well as 
thermoelectric materials can be developed, paving the way towards novel applications based on molecule-TMD hybrids.

\section{Conclusions and outlook}

The large variety of materials/properties available in the TMD family combined with the virtually-infinite number of functional molecular systems available through chemical synthesis provide countless opportunities to engineer hybrid organic/inorganic nanomaterials with on-demand characteristics for device applications. In such hybrids, new functionalities can be introduced in a chemically controlled manner by tailoring the interactions between molecules ultrathin crystalline materials, ${ }^{199}$ thereby opening further possibilities for developing multifunctional/multiresponsive materials and devices. Hereafter, we will discuss these newly emerging research fronts, as well as the prospects and the challenges towards next-generation technologies based on molecule-TMD hybrid materials.

Hybrid van der Waals heterostructures. The molecular chemistry approaches to TMDs present several analogies to the quest for materials by design carried out by superimposing different inorganic 2D materials in the so-called van der Waals heterostructures (Fig. 19a). ${ }^{419 \text {, }}$

${ }^{420}$ Molecules can be incorporated into these layered structures to generate novel hybrid materials in which unique molecular capabilities are combined with the ultra-high electrical performances of TMDs and other 2D materials. Towards this goal, molecular science offers a variety of opportunities which have not yet been fully exploited.

So far, relatively few studies exploit the possibility to generate highly predictable selfassembled molecular monolayers on the surface of TMDs ${ }^{219,252,253,421,422}$ and impart them on-demand functions. Indeed, the molecular arrangement on TMD surfaces is determined by supramolecular interactions which can be predicted and controlled by molecular design. Mastering molecular self-assembly offers the possibility to incorporate specific functional 
groups (such as dopants) at pre-determined spatial locations, paving the way to a direct correlation between electrical effects measured at the device level and events taking place at the molecular scale. Moreover, such molecular chemistry approaches ensure an atomic precision that outperforms conventional lithographic techniques, ${ }^{423-425}$ and molecular monolayers self-align with respect to the crystalline direction of the underlying substrate giving rise to epitaxial growth. ${ }^{426,427}$ On graphene, this approach was exploited to induce controllable periodic potentials ${ }^{93}$ and demonstrate tunable doping. ${ }^{428}$

The same ultra-high control over the nanoscale molecular ordering can be achieved in the case of TMDs. ${ }^{81}$ Recently, Wang et al. ${ }^{252}$ demonstrated that oleamide generates analogous crystalline structures on the surface of different 2D materials, including several TMDs $\mathrm{MoS}_{2}, \mathrm{MoSe}_{2}, \mathrm{MoTe}_{2}, \mathrm{WSe}_{2}$ (Fig. 19 b-d). Within the SAMs, oleamide molecules lie flat on the substrate surface, and intermolecular hydrogen bonds drive the formation of parallel rows, which are aligned to the crystallographic orientation of the $2 \mathrm{D}$ materials (Fig.16e). In this regard, the growth is epitaxial and atomically precise, with single crystalline molecular domains extending over regions above $0.5 \mu \mathrm{m} \times 0.5 \mu \mathrm{m}$ (Fig.16 b-d). ${ }^{252}$ Whether the structural 1D anisotropy of the assembly induces anisotropy in the electronic properties of the underlying TMD remains an open question, which can only be answered by an investigation of electrical transport and optical characteristics of regions covered by a single crystalline molecular domain. In turn, this goal poses the challenge of maximizing the size of molecular crystalline domains, and of fabricating nanoscale device which preserve the molecular monolayers. Finally, we highlight that molecular decoration affects not only the optoelectronic, but also the magnetic properties of TMDs, ${ }^{114,429}$ opening interesting avenues for spintronics based on $\mathrm{TMD} /$ molecules heterostructures. In perspective, the ability to generate heterostructures with engineered properties can be exploited to build up a library of novel hybrid materials with on-demand properties. 
Multifunctional materials and devices. Combining molecular systems with ultrathin TMDs allows not only to control the charge-carrier doping of $2 \mathrm{D}$ semiconductors but also to modulate their optical, thermal and sensing properties in hybrid systems. Such an approach makes it also possible to impart a multifunctional/multiresponsive nature to $2 \mathrm{D}$ TMDs that can be conveniently exploited for developing multifunctional (opto)electronic devices, which can be controlled with multiple independent stimuli. For instance, by making use of the different molecular chemistry methods discussed in this review, 2D sheets of TMDs could be functionalized with molecules carrying photochromic, ${ }^{430-432}$ magneto-responsive ${ }^{433}$ and electrochemically-switchable ${ }^{434,435}$ moieties. External stimuli, such as heat, light, magnetic fields and electrochemical signals, can then be used to trigger changes in the structures/properties of the molecules interfacing the ultrathin sheets. Thanks to their ultimately-large surface to volume ratio, ultrathin TMDs are expected to respond to such changes with variations in their electrical, optical and magnetic characteristics. One interesting example has been recently provided by Datta et al., ${ }^{429}$ who developed FETs based on monolayer $\mathrm{MoS}_{2}$ sheets "decorated" on the top surface with magnetic molecules, such as quinoidal dithienyl perylenequinodimethane (QDTP). The latter undergo a spin transition from a singlet to a triplet state with increasing temperature above $\sim 370 \mathrm{~K}$. This spin-state switch could be electrically transduced by the $\mathrm{MoS}_{2}$ sheet, which displayed an increase in the free electron density ( $n$ doping) and a strong enhancement in magnetoconductance $(\sim 100 \%)$ above the magnetic transition temperature. This work demonstrated that the electrical/magnetic properties of monolayer $\mathrm{MoS}_{2}$ could be dynamically modified through the interaction with switchable molecular systems. In the next years, further research is envisioned towards multifunctional/multiresponsive materials and devices based on combinations of ultrathin TMDs and molecular switches. 
Next challenges. In order to take full advantage of TMD-molecule hybrids in technological applications, various challenges have to be addressed. Besides the aforementioned requirement to control/enlarge the size of physisorbed molecular crystal domains, an important task towards hybrid TMD-molecule (opto)electronics consists in developing suitable techniques (e.g. encapsulation) to prevent desorption or decomposition of molecules during device operation. It is also of paramount importance to achieve a systematic control over the degree of functionalization in both solution-processed and substrate-supported 2D sheets. The latter should be carefully quantified through multiple experimental techniques and must be systematically controlled, especially in the case of optoelectronic devices and sensing technologies, where uniform and reproducible optical, electronic and sensing properties must be achieved across the entire active material's surface. However, such requirement might not be so stringent for multifunctional foams and composites, ${ }^{436}$ or for biomedical applications, ${ }^{198}$ which could be the first areas where hybrid molecule-TMDs could find practical application, in order to address societal needs in energy generation and storage as well as water purification and highly selective gas and ion sensors. Towards the improvement of the quality of our lives, in the forthcoming years the development of structurally defined, novel hybrid materials based on TMDs and suitably designed molecules, shall open a new technological era relying on portable, flexible and foldable multiresponsive (opto)electronic devices for health and environmental monitoring. The full exploitation of the infinite options offered by molecular science in terms of structural and functional diversities shall soon yield to the emergence of new, disruptive and exotic technologies based on TMDs hybrids.

\section{Conflict of interest}

There are no conflicts to declare. 


\section{Acknowledgments}

We acknowledge funding from the European Commission through the Graphene Flagship (GA-696656), the Marie-Curie IEF MULTI2DSWITCH (GA-700802) and the Marie Curie IEF SUPER2D (GA-748971), the M-ERA.NET project MODIGLIANI, the Agence Nationale de la Recherche through the LabEx projects CSC (ANR-10-LABX-0026 CSC) and Nanostructures in Interaction with their Environment (ANR-11-LABX-0058 NIE) within the Investissement d'Avenir program (ANR-10-120 IDEX-0002-02), and the International Center for Frontier Research in Chemistry (icFRC). C.B. acknowledges the German research foundation DFG under Emmy-Noether grant BA4856/2-1. 


\section{References}

1. D. L. Duong, S. J. Yun and Y. H. Lee, ACS Nano, 2017, 11, 11803.

2. P. Miro, M. Audiffred and T. Heine, Chem. Soc. Rev., 2014, 43, 6537.

3. A. C. Ferrari, F. Bonaccorso, V. Fal'ko, K. S. Novoselov, S. Roche, P. Boggild, S. Borini, F. H. L. Koppens, V. Palermo, N. Pugno, J. A. Garrido, R. Sordan, A. Bianco, L. Ballerini, M. Prato, E. Lidorikis, J. Kivioja, C. Marinelli, T. Ryhanen, A. Morpurgo, J. N. Coleman, V. Nicolosi, L. Colombo, A. Fert, M. Garcia-Hernandez, A. Bachtold, G. F. Schneider, F. Guinea, C. Dekker, M. Barbone, Z. P. Sun, C. Galiotis, A. N. Grigorenko, G. Konstantatos, A. Kis, M. Katsnelson, L. Vandersypen, A. Loiseau, V. Morandi, D. Neumaier, E. Treossi, V. Pellegrini, M. Polini, A. Tredicucci, G. M. Williams, B. H. Hong, J. H. Ahn, J. M. Kim, H. Zirath, B. J. van Wees, H. van der Zant, L. Occhipinti, A. Di Matteo, I. A. Kinloch, T. Seyller, E. Quesnel, X. L. Feng, K. Teo, N. Rupesinghe, P. Hakonen, S. R. T. Neil, Q. Tannock, T. Lofwander and J. Kinaret, Nanoscale, 2015, 7, 4598.

4. K. S. Novoselov, A. K. Geim, S. V. Morozov, D. Jiang, Y. Zhang, S. V. Dubonos, I. V. Grigorieva and A. A. Firsov, Science, 2004, 306, 666.

5. K. S. Novoselov, D. Jiang, F. Schedin, T. J. Booth, V. V. Khotkevich, S. V. Morozov and A. K. Geim, Proc. Natl. Acad. Sci. USA, 2005, 102, 10451.

6. A. K. Geim and K. S. Novoselov, Nat. Mater., 2007, 6, 183.

7. F. Schwierz, Nat. Nanotechnol., 2010, 5, 487.

8. A. Kuc and T. Heine, Chem. Soc. Rev., 2015, 44, 2603.

9. S. Manzeli, D. Ovchinnikov, D. Pasquier, O. V. Yazyev and A. Kis, Nat. Rev. Mat., 2017, 2, 17033 .

10. R. Fivaz and E. Mooser, Phys. Rev., 1967, 163, 743.

11. J. A. Wilson and A. D. Yoffe, Adv. Phys., 1969, 18, 193.

12. R. F. Frindt and A. D. Yoffe, Proc. R. Soc. London, Ser. A, 1963, 273, 69.

13. R. F. Frindt, J. Appl. Phys., 1966, 37, 1928.

14. P. Joensen, R. F. Frindt and S. R. Morrison, Mater. Res. Bull., 1986, 21, 457.

15. Y. Jung, Y. Zhou and J. J. Cha, Inorg. Chem. Front., 2016, 3, 452.

16. J. R. Brent, N. Savjani and P. O'Brien, Prog. Mater. Sci., 2017, 89, 411.

17. G. R. Bhimanapati, Z. Lin, V. Meunier, Y. Jung, J. Cha, S. Das, D. Xiao, Y. Son, M. S. Strano, V. R. Cooper, L. Liang, S. G. Louie, E. Ringe, W. Zhou, S. S. Kim, R. R. Naik, B. G. Sumpter, H. Terrones, F. Xia, Y. Wang, J. Zhu, D. Akinwande, N. Alem, J. A. Schuller, R. E. Schaak, M. Terrones and J. A. Robinson, ACS Nano, 2015, 9, 11509.

18. W. Choi, N. Choudhary, G. H. Han, J. Park, D. Akinwande and Y. H. Lee, Mater. Today, 2017, 20, 116.

19. O. V. Yazyev and A. Kis, Mater. Today, 2015, 18, 20.

20. B. Radisavljevic, A. Radenovic, J. Brivio, V. Giacometti and A. Kis, Nat. Nanotechnol., $2011,6,147$.

21. M. S. Fuhrer and J. Hone, Nat. Nanotechnol., 2013, 8, 146.

22. B. Radisavljevic and A. Kis, Nat. Nanotechnol., 2013, 8, 147.

23. G. Fiori, F. Bonaccorso, G. Iannaccone, T. Palacios, D. Neumaier, A. Seabaugh, S. K. Banerjee and L. Colombo, Nat. Nanotechnol., 2014, 9, 768.

24. D. Lembke, S. Bertolazzi and A. Kis, Acc. Chem. Res., 2015, 48, 100.

25. H. Schmidt, F. Giustiniano and G. Eda, Chem. Soc. Rev., 2015, 44, 7715.

26. D. Jariwala, V. K. Sangwan, L. J. Lauhon, T. J. Marks and M. C. Hersam, ACS Nano, $2014,8,1102$.

27. Q. H. Wang, K. Kalantar-Zadeh, A. Kis, J. N. Coleman and M. S. Strano, Nat. Nanotechnol., 2012, 7, 699.

28. L. T. Liu, Y. Lu and J. Guo, IEEE Trans. Electron. Devices, 2013, 60, 4133.

29. K. Alam and R. K. Lake, IEEE Trans. Electron. Devices, 2012, 59, 3250.

30. M. C. Lemme, in Beyond-CMOS Nanodevices 2, ed. F. Balestra, ISTE Ltd, London, John Wiley \& Sons Inc., Hoboken, New Jersey, 2014, ch. 3, pp. 97.

31. M. M. Waldrop, Nature, 2016, 530, 144.

32. A. Kuc, N. Zibouche and T. Heine, Phys. Rev., B, 2011, 83, 245213.

33. A. Splendiani, L. Sun, Y. B. Zhang, T. S. Li, J. Kim, C. Y. Chim, G. Galli and F. Wang, Nano Lett., 2010, 10, 1271. 
34. K. F. Mak, C. Lee, J. Hone, J. Shan and T. F. Heinz, Phys. Rev. Lett., 2010, 105.

35. K. F. Mak and J. Shan, Nat. Photonics, 2016, 10, 216.

36. J. Shim, H.-Y. Park, D.-H. Kang, J.-O. Kim, S.-H. Jo, Y. Park and J.-H. Park, Adv. Electron. Mater., 2017, 3, 1600364.

37. B. W. H. Baugher, H. O. H. Churchill, Y. F. Yang and P. Jarillo-Herrero, Nat. Nanotechnol., 2014, 9, 262.

38. F. H. L. Koppens, T. Mueller, P. Avouris, A. C. Ferrari, M. S. Vitiello and M. Polini, Nat. Nanotechnol., 2014, 9, 780.

39. D. H. Kang, M. S. Kim, J. Shim, J. Jeon, H. Y. Park, W. S. Jung, H. Y. Yu, C. H. Pang, S. Lee and J. H. Park, Adv. Funct. Mater., 2015, 25, 4219.

40. O. Lopez-Sanchez, D. Lembke, M. Kayci, A. Radenovic and A. Kis, Nat. Nanotechnol., 2013, 8, 497.

41. A. Pospischil, M. M. Furchi and T. Mueller, Nat. Nanotechnol., 2014, 9, 257.

42. M. Bernardi, M. Palummo and J. C. Grossman, Nano Lett., 2013, 13, 3664.

43. L. Britnell, R. M. Ribeiro, A. Eckmann, R. Jalil, B. D. Belle, A. Mishchenko, Y. J. Kim, R. V. Gorbachev, T. Georgiou, S. V. Morozov, A. N. Grigorenko, A. K. Geim, C. Casiraghi, A. H. Castro Neto and K. S. Novoselov, Science, 2013, 340, 1311.

44. P. Huang, Z. Wang, Y. Liu, K. Zhang, L. Yuan, Y. Zhou, B. Song and Y. Li, ACS. Appl. Mater. Interfaces, 2017, 9, 25323.

45. S. Bertolazzi, J. Brivio and A. Kis, ACS Nano, 2011, 5, 9703.

46. D. Akinwande, C. J. Brennan, J. S. Bunch, P. Egberts, J. R. Felts, H. Gao, R. Huang, J.-S. Kim, T. Li, Y. Li, K. M. Liechti, N. Lu, H. S. Park, E. J. Reed, P. Wang, B. I. Yakobson, T. Zhang, Y.-W. Zhang, Y. Zhou and Y. Zhu, Extreme Mech. Lett., 2017, 13, 42.

47. S. Das, R. Gulotty, A. V. Sumant and A. Roelofs, Nano Lett., 2014, 14, 2861.

48. R. Cheng, S. Jiang, Y. Chen, Y. Liu, N. Weiss, H. C. Cheng, H. Wu, Y. Huang and X. F. Duan, Nat. Commun., 2014, 5, 5143.

49. A. Nathan, A. Ahnood, M. T. Cole, S. Lee, Y. Suzuki, P. Hiralal, F. Bonaccorso, T. Hasan, L. Garcia-Gancedo, A. Dyadyusha, S. Haque, P. Andrew, S. Hofmann, J. Moultrie, D. P. Chu, A. J. Flewitt, A. C. Ferrari, M. J. Kelly, J. Robertson, G. A. J. Amaratunga and W. I. Milne, Proc. IEEE, 2012, 100, 1486.

50. Y. M. Shi, H. N. Li and L. J. Li, Chem. Soc. Rev., 2015, 44, 2744.

51. Y. D. Zhao, K. Xu, F. Pan, C. J. Zhou, F. C. Zhou and Y. Chai, Adv. Funct. Mater., 2017, 27, 1603484.

52. N. Onofrio, D. Guzman and A. Strachan, J. Appl. Phys., 2017, 122, 185102.

53. V. P. Pham and G. Y. Yeom, Adv. Mater., 2016, 28, 9024.

54. Y. H. Lee, X. Q. Zhang, W. J. Zhang, M. T. Chang, C. T. Lin, K. D. Chang, Y. C. Yu, J. T. W. Wang, C. S. Chang, L. J. Li and T. W. Lin, Adv. Mater., 2012, 24, 2320.

55. Y. J. Zhan, Z. Liu, S. Najmaei, P. M. Ajayan and J. Lou, Small, 2012, 8, 966.

56. A. M. van der Zande, P. Y. Huang, D. A. Chenet, T. C. Berkelbach, Y. M. You, G. H. Lee, T. F. Heinz, D. R. Reichman, D. A. Muller and J. C. Hone, Nat. Mater., 2013, 12, 554.

57. S. M. Sze and K. K. Ng, Physics of Semiconductor Devices, John Wiley \& Sons Inc., Hoboken, New Jersey, 2006.

58. G. S. May and C. J. Spanos, Fundamentals of semiconductor manufacturing and process control, Wiley-Interscience, Hoboken, New Jersey, 2006.

59. K. Haynes, R. Murray, Z. Weinrich, X. Zhao, D. Chiappe, S. Sutar, I. Radu, C. Hatem, S. S. Perry and K. S. Jones, Appl. Phys. Lett., 2017, 110, 262102.

60. R. Murray, K. Haynes, X. Y. Zhao, S. Perry, C. Hatem and K. Jones, ECS J. Solid State Sci. Technol., 2016, 5, Q3050.

61. A. Nipane, D. Karmakar, N. Kaushik, S. Karande and S. Lodha, ACS Nano, 2016, 10, 2128.

62. M. Zhang, J. X. Wu, Y. M. Zhu, D. O. Dumcenco, J. H. Hong, N. N. Mao, S. B. Deng, Y. F. Chen, Y. L. Yang, C. H. Jin, S. H. Chaki, Y. S. Huang, J. Zhang and L. M. Xie, ACS Nano, $2014,8,7130$.

63. A. A. Tedstone, D. J. Lewis and P. O'Brien, Chem. Mater., 2016, 28, 1965.

64. J. Gao, Y. D. Kim, L. Liang, J. C. Idrobo, P. Chow, J. Tan, B. Li, L. Li, B. G. Sumpter, T.-M. Lu, V. Meunier, J. Hone and N. Koratkar, Adv. Mater., 2016, 28, 9735.

65. J. Suh, T.-E. Park, D.-Y. Lin, D. Fu, J. Park, H. J. Jung, Y. Chen, C. Ko, C. Jang, Y. Sun, R. Sinclair, J. Chang, S. Tongay and J. Wu, Nano Lett., 2014, 14, 6976. 
66. Y. C. Tsai and Y. Li, IEEE Trans. Electron Devices, 2018, 65, 733.

67. X. G. Liang, H. S. Nam, S. J. Wi and M. K. Chen, 2014 25th Annual SEMI Advanced Semiconductor Manufacturing Conference (ASMC), 2014, 365.

68. H.-P. Komsa, J. Kotakoski, S. Kurasch, O. Lehtinen, U. Kaiser and A. V. Krasheninnikov, Phys. Rev. Lett., 2012, 109, 035503.

69. Z. Lin, B. R. Carvalho, E. Kahn, R. T. Lv, R. Rao, H. Terrones, M. A. Pimenta and M. Terrones, 2D Mater., 2016, 3, 022002.

70. H. T. Wang, H. T. Yuan, S. S. Hong, Y. B. Li and Y. Cui, Chem. Soc. Rev., 2015, 44, 2664.

71. F. K. Perkins, A. L. Friedman, E. Cobas, P. M. Campbell, G. G. Jernigan and B. T. Jonker, Nano Lett., 2013, 13, 668.

72. W. Yang, L. Gan, H. Li and T. Zhai, Inorg. Chem. Front., 2016, 3, 433.

73. T. Kim, Y. Kim, S. Park, S. Kim and H. Jang, Chemosensors, 2017, 5, 15.

74. A. Bandyopadhyay and S. K. Pati, Mater. Res. Express, 2015, 2, 085003.

75. C. R. Ryder, J. D. Wood, S. A. Wells and M. C. Hersam, ACS Nano, 2016, 10, 3900.

76. S. Presolski and M. Pumera, Mater. Today, 2016, 19, 140.

77. Q. Tang and D. E. Jiang, Chem. Mater., 2015, 27, 3743.

78. P. Vishnoi, A. Sampath, U. V. Waghmare and C. N. R. Rao, Chem. Eur. J., 2017, 23, 886.

79. P. D. Zhao, D. Kiriya, A. Azcatl, C. X. Zhang, M. Tosun, Y. S. Liu, M. Hettick, J. S.

Kang, S. McDonnell, K. C. Santosh, J. H. Guo, K. Cho, R. M. Wallace and A. Javey, ACS Nano, 2014, 8, 10808.

80. D. Voiry, A. Goswami, R. Kappera, C. D. C. C. E. Silva, D. Kaplan, T. Fujita, M. Chen, T. Asefa and M. Chhowalla, Nat. Chem., 2015, 7, 45.

81. C. J. L. de la Rosa, R. Phillipson, J. Teyssandier, J. Adisoejoso, Y. Balaji, C. Huyghebaert, I. Radu, M. Heyns, S. De Feyter and S. De Gendt, Appl. Phys. Lett., 2016, 109, 253112.

82. Y. Jing, X. Tan, Z. Zhou and P. W. Shen, J. Mater. Chem. A, 2014, 2, 16892.

83. A. Kumar, K. Banerjee and P. Liljeroth, Nanotechnology, 2017, 28, 082001.

84. Y. Li, C.-Y. Xu, P. Hu and L. Zhen, ACS Nano, 2013, 7, 7795.

85. D. Kiriya, M. Tosun, P. D. Zhao, J. S. Kang and A. Javey, J. Am. Chem. Soc., 2014, 136, 7853.

86. D.-H. Kang, J. Shim, S. K. Jang, J. Jeon, M. H. Jeon, G. Y. Yeom, W.-S. Jung, Y. H. Jang, S. Lee and J.-H. Park, ACS Nano, 2015, 9, 1099.

87. A. Tarasov, S. Y. Zhang, M. Y. Tsai, P. M. Campbell, S. Graham, S. Barlow, S. R. Marder and E. M. Vogel, Adv. Mater., 2015, 27, 1175.

88. D. M. Sim, M. Kim, S. Yim, M.-J. Choi, J. Choi, S. Yoo and Y. S. Jung, ACS Nano, 2015, 9, 12115.

89. S. N. Zhang, C. J. Benjamin and Z. Chen, presented in part at the 75th Annual Device Research Conference, South Bend, IN, USA, June, 2017.

90. H. Y. Park, S. R. Dugasani, D. H. Kang, J. Jeon, S. K. Jang, S. Lee, Y. Roh, S. H. Park and J. H. Park, ACS Nano, 2014, 8, 11603.

91. T. Kawanago and S. Oda, Appl. Phys. Lett., 2016, 108, 041605.

92. S. Najmaei, X. L. Zou, D. Q. Er, J. W. Li, Z. H. Jin, W. L. Gao, Q. Zhang, S. Park, L. H. Ge, S. D. Lei, J. Kono, V. B. Shenoy, B. I. Yakobson, A. George, P. M. Ajayan and J. Lou, Nano Lett., 2014, 14, 1354.

93. M. Gobbi, S. Bonacchi, J. X. Lian, Y. Liu, X.-Y. Wang, M.-A. Stoeckel, M. A. Squillaci, G. D'Avino, A. Narita, K. Müllen, X. Feng, Y. Olivier, D. Beljonne, P. Samorì and E. Orgiu, Nat. Commun., 2017, 8, 14767.

94. Y. C. Du, H. Liu, A. T. Neal, M. W. Si and P. D. Ye, IEEE Electron. Dev. Lett., 2013, 34, 1328.

95. D. Çakır, C. Sevik, F. M. Peeters, J. Li, Y. Shi, X. Wang, P. Barbara, M. Pan, D. Xaio, J. Yan, D. Mandrus and Z. Zhou, J. Mater. Chem. C, 2014, 2, 9842.

96. M. Amani, P. Taheri, R. Addou, G. H. Ahn, D. Kiriya, D. H. Lien, J. W. Ager, R. M. Wallace and A. Javey, Nano Lett., 2016, 16, 2786.

97. M. Amani, D.-H. Lien, D. Kiriya, J. Xiao, A. Azcatl, J. Noh, S. R. Madhvapathy, R. Addou, S. Kc, M. Dubey, K. Cho, R. M. Wallace, S.-C. Lee, J.-H. He, J. W. Ager, X. Zhang, E. Yablonovitch and A. Javey, Science, 2015, 350, 1065. 
98. M. Amani, R. A. Burke, X. Ji, P. Zhao, D. H. Lien, P. Taheri, G. H. Ahn, D. Kirya, J. W. Ager, E. Yablonovitch, J. Kong, M. Dubey and A. Javey, ACS Nano, 2016, 10, 6535.

99. J. Choi, H. Zhang and J. H. Choi, ACS Nano, 2016, 10, 1671.

100. H. V. Han, A. Y. Lu, L. S. Lu, J. K. Huang, H. Li, C. L. Hsu, Y. C. Lin, M. H. Chiu, K. Suenaga, C. W. Chu, H. C. Kuo, W. H. Chang, L. J. Li and Y. Shi, ACS Nano, 2016, 10, 1454.

101. S.-H. Jo, H.-Y. Park, D.-H. Kang, J. Shim, J. Jeon, S. Choi, M. Kim, Y. Park, J. Lee, Y. J. Song, S. Lee and J.-H. Park, Adv. Mater., 2016, 28, 6711.

102. W. Su, H. Dou, D. Huo, N. Dai and L. Yang, Chem. Phys. Lett., 2015, 635, 40.

103. S. Tongay, J. Zhou, C. Ataca, J. Liu, J. S. Kang, T. S. Matthews, L. You, J. Li, J. C. Grossman and J. Wu, Nano Lett., 2013, 13, 2831.

104. M. Ayán-Varela, Ó. Pérez-Vidal, J. I. Paredes, J. M. Munuera, S. Villar-Rodil, M. Díaz-González, C. Fernández-Sánchez, V. S. Silva, M. Cicuéndez, M. Vila, A. MartínezAlonso and J. M. D. Tascón, ACS. Appl. Mater. Interfaces, 2017, 9, 2835.

105. E. Varrla, C. Backes, K. R. Paton, A. Harvey, Z. Gholamvand, J. McCauley and J. N. Coleman, Chem. Mater., 2015, 27, 1129.

106. F. Bonaccorso, A. Bartolotta, J. N. Coleman and C. Backes, Adv. Mater., 2016, 28, 6136.

107. A. G. Kelly, T. Hallam, C. Backes, A. Harvey, A. S. Esmaeily, I. Godwin, J. Coelho, V. Nicolosi, J. Lauth, A. Kulkarni, S. Kinge, L. D. A. Siebbeles, G. S. Duesberg and J. N. Coleman, Science, 2017, 356, 69.

108. D. McManus, S. Vranic, F. Withers, V. Sanchez-Romaguera, M. Macucci, H. Yang, R. Sorrentino, K. Parvez, S.-K. Son, G. Iannaccone, K. Kostarelos, G. Fiori and C. Casiraghi, Nat. Nanotechnol., 2017, 12, 343.

109. S. Bertolazzi, S. Bonacchi, G. J. Nan, A. Pershin, D. Beljonne and P. Samorì, Adv. Mater., 2017, 29, 1606760.

110. C. Backes, N. C. Berner, X. Chen, P. Lafargue, P. LaPlace, M. Freeley, G. S. Duesberg, J. N. Coleman and A. R. McDonald, Angew. Chem. Int. Ed., 2015, 54, 2638.

111. X. Chen and A. R. McDonald, Adv. Mater., 2016, 28, 5738.

112. E. P. Nguyen, B. J. Carey, J. Z. Ou, J. van Embden, E. D. Gaspera, A. F. Chrimes, M. J. S. Spencer, S. Zhuiykov, K. Kalantar-zadeh and T. Daeneke, Adv. Mater., 2015, 27, 6225.

113. W. L. Chow, P. Yu, F. Liu, J. Hong, X. Wang, Q. Zeng, C.-H. Hsu, C. Zhu, J. Zhou, X. Wang, J. Xia, J. Yan, Y. Chen, D. Wu, T. Yu, Z. Shen, H. Lin, C. Jin, B. K. Tay and Z. Liu, Adv. Mater., 2017, 29, 1602969.

114. X. Zhu, Y. Guo, H. Cheng, J. Dai, X. An, J. Zhao, K. Tian, S. Wei, X. Cheng Zeng, C. Wu and Y. Xie, Nat. Commun., 2016, 7, 11210.

115. M. Chhowalla, H. S. Shin, G. Eda, L. J. Li, K. P. Loh and H. Zhang, Nat. Chem., 2013, 5, 263.

116. N. Mounet, M. Gibertini, P. Schwaller, D. Campi, A. Merkys, A. Marrazzo, T. Sohier, I. E. Castelli, A. Cepellotti, G. Pizzi and N. Marzari, Nat. Nanotechnol., 2018, DOI: $10.1038 / \mathrm{s} 41565$.

117. M. B. Dines, Mater. Res. Bull., 1975, 10, 287.

118. Z. Y. Zeng, Z. Y. Yin, X. Huang, H. Li, Q. Y. He, G. Lu, F. Boey and H. Zhang, Angew. Chem. Int. Ed., 2011, 50, 11093.

119. J. Zheng, H. Zhang, S. Dong, Y. Liu, C. Tai Nai, H. Suk Shin, H. Young Jeong, B. Liu and K. Ping Loh, Nat. Commun., 2014, 5, 2995.

120. J. Peng, J. Wu, X. Li, Y. Zhou, Z. Yu, Y. Guo, J. Wu, Y. Lin, Z. Li, X. Wu, C. Wu and Y. Xie, J. Am. Chem. Soc., 2017, 139, 9019.

121. R. A. Gordon, D. Yang, E. D. Crozier, D. T. Jiang and R. F. Frindt, Phys. Rev., B, 2002, 65, 125407.

122. J. N. Coleman, M. Lotya, A. O’Neill, S. D. Bergin, P. J. King, U. Khan, K. Young, A. Gaucher, S. De, R. J. Smith, I. V. Shvets, S. K. Arora, G. Stanton, H.-Y. Kim, K. Lee, G. T. Kim, G. S. Duesberg, T. Hallam, J. J. Boland, J. J. Wang, J. F. Donegan, J. C. Grunlan, G. Moriarty, A. Shmeliov, R. J. Nicholls, J. M. Perkins, E. M. Grieveson, K. Theuwissen, D. W. McComb, P. D. Nellist and V. Nicolosi, Science, 2011, 331, 568.

123. V. Nicolosi, M. Chhowalla, M. G. Kanatzidis, M. S. Strano and J. N. Coleman, Science, 2013, 340, 1420. 
124. X. S. Li, W. W. Cai, J. H. An, S. Kim, J. Nah, D. X. Yang, R. Piner, A. Velamakanni, I. Jung, E. Tutuc, S. K. Banerjee, L. Colombo and R. S. Ruoff, Science, 2009, 324, 1312.

125. Y. F. Hao, M. S. Bharathi, L. Wang, Y. Y. Liu, H. Chen, S. Nie, X. H. Wang, H. Chou, C. Tan, B. Fallahazad, H. Ramanarayan, C. W. Magnuson, E. Tutuc, B. I. Yakobson, K. F. McCarty, Y. W. Zhang, P. Kim, J. Hone, L. Colombo and R. S. Ruoff, Science, 2013, 342, 720 .

126. D. Dumcenco, D. Ovchinnikov, K. Marinov, P. Lazic, M. Gibertini, N. Marzari, O. L. Sanchez, Y. C. Kung, D. Krasnozhon, M. W. Chen, S. Bertolazzi, P. Gillet, A. F. I. Morral, A. Radenovic and A. Kis, ACS Nano, 2015, 9, 4611.

127. S. L. Wong, H. F. Liu and D. Z. Chi, Prog. Cryst. Growth Charact. Mater., 2016, 62, 9.

128. J. Yu, J. Li, W. Zhang and H. Chang, Chem. Sci., 2015, 6, 6705.

129. K. Keyshar, Y. J. Gong, G. L. Ye, G. Brunetto, W. Zhou, D. P. Cole, K. Hackenberg, Y. M. He, L. Machado, M. Kabbani, A. H. C. Hart, B. Li, D. S. Galvao, A. George, R. Vajtai, C. S. Tiwary and P. M. Ajayan, Adv. Mater., 2015, 27, 4640.

130. X. Zhou, L. Gan, W. M. Tian, Q. Zhang, S. Y. Jin, H. Q. Li, Y. Bando, D. Golberg and T. Y. Zhai, Adv. Mater., 2015, 27, 8035.

131. X. Q. Zhang, C. H. Lin, Y. W. Tseng, K. H. Huang and Y. H. Lee, Nano Lett., 2015, $15,410$.

132. C. Tan and H. Zhang, J. Am. Chem. Soc., 2015, 137, 12162.

133. M. Kertesz and R. Hoffmann, J. Am. Chem. Soc., 1984, 106, 3453.

134. K.-A. N. Duerloo, Y. Li and E. J. Reed, Nat. Commun., 2014, 5, 4214.

135. H. Yang, S. W. Kim, M. Chhowalla and Y. H. Lee, Nat. Phys., 2017, 13, 931.

136. C. H. Naylor, W. M. Parkin, J. Ping, Z. Gao, Y. R. Zhou, Y. Kim, F. Streller, R. W. Carpick, A. M. Rappe, M. Drndić, J. M. Kikkawa and A. T. C. Johnson, Nano Lett., 2016, 16, 4297.

137. Y. Qi, P. G. Naumov, M. N. Ali, C. R. Rajamathi, W. Schnelle, O. Barkalov, M. Hanfland, S.-C. Wu, C. Shekhar, Y. Sun, V. Süß, M. Schmidt, U. Schwarz, E. Pippel, P. Werner, R. Hillebrand, T. Förster, E. Kampert, S. Parkin, R. J. Cava, C. Felser, B. Yan and S. A. Medvedev, Nat. Commun., 2016, 7, 11038.

138. C. Ataca, H. Sahin and S. Ciraci, J. Phys. Chem. C, 2012, 116, 8983.

139. F. A. Rasmussen and K. S. Thygesen, J. Phys. Chem. C, 2015, 119, 13169.

140. S. J. Sandoval, D. Yang, R. F. Frindt and J. C. Irwin, Phys. Rev., B, 1991, 44, 3955.

141. G. Eda, T. Fujita, H. Yamaguchi, D. Voiry, M. Chen and M. Chhowalla, ACS Nano, $2012,6,7311$.

142. G. Eda, H. Yamaguchi, D. Voiry, T. Fujita, M. Chen and M. Chhowalla, Nano Lett., $2011,11,5111$.

143. K. Leng, Z. Chen, X. Zhao, W. Tang, B. Tian, C. T. Nai, W. Zhou and K. P. Loh, ACS Nano, 2016, 10, 9208.

144. Y. Zhao, J. Qiao, Z. Yu, P. Yu, K. Xu, S. P. Lau, W. Zhou, Z. Liu, X. Wang, W. Ji and Y. Chai, Adv. Mater., 2017, 29, 1604230.

145. Y. Cao, A. Mishchenko, G. L. Yu, E. Khestanova, A. P. Rooney, E. Prestat, A. V. Kretinin, P. Blake, M. B. Shalom, C. Woods, J. Chapman, G. Balakrishnan, I. V. Grigorieva, K. S. Novoselov, B. A. Piot, M. Potemski, K. Watanabe, T. Taniguchi, S. J. Haigh, A. K. Geim and R. V. Gorbachev, Nano Lett., 2015, 15, 4914.

146. S. Cho, S. Kim, J. H. Kim, J. Zhao, J. Seok, D. H. Keum, J. Baik, D.-H. Choe, K. J. Chang, K. Suenaga, S. W. Kim, Y. H. Lee and H. Yang, Science, 2015, 349, 625.

147. Y. Wang, J. Xiao, H. Zhu, Y. Li, Y. Alsaid, K. Y. Fong, Y. Zhou, S. Wang, W. Shi, Y. Wang, A. Zettl, E. J. Reed and X. Zhang, Nature, 2017, 550, 487.

148. Y. C. Lin, H. P. Komsa, C. H. Yeh, T. Bjorkman, Z. Y. Liang, C. H. Ho, Y. S. Huang, P. W. Chiu, A. V. Krasheninnikov and K. Suenaga, ACS Nano, 2015, 9, 11249.

149. D. Ovchinnikov, F. Gargiulo, A. Allain, D. J. Pasquier, D. Dumcenco, C. H. Ho, O. V. Yazyev and A. Kis, Nat. Commun., 2016, 7, 12391.

150. T. Heine, Acc. Chem. Res., 2015, 48, 65.

151. P. Y. Yu and C. Manuel, Fundamentals of Semiconductors: Physics and Materials Properties, Springer, London, 2010. 
152. C. Chakraborty, L. Kinnischtzke, K. M. Goodfellow, R. Beams and A. N. Vamivakas, Nat. Nanotechnol., 2015, 10, 507.

153. D. W. Boukhvalov and M. I. Katsnelson, Nano Lett., 2008, 8, 4373.

154. M. Yi and Z. G. Shen, J. Mater. Chem. A, 2015, 3, 11700.

155. M. Eredia, S. Bertolazzi, T. Leydecker, M. El Garah, I. Janica, G. Melinte, O. Ersen, A. Ciesielski and P. Samorì, J. Phys. Chem. Lett., 2017, 8, 3347.

156. P. Y. Huang, C. S. Ruiz-Vargas, A. M. van der Zande, W. S. Whitney, M. P. Levendorf, J. W. Kevek, S. Garg, J. S. Alden, C. J. Hustedt, Y. Zhu, J. Park, P. L. McEuen and D. A. Muller, Nature, 2011, 469, 389.

157. H. Qiu, T. Xu, Z. Wang, W. Ren, H. Nan, Z. Ni, Q. Chen, S. Yuan, F. Miao, F. Song, G. Long, Y. Shi, L. Sun, J. Wang and X. Wang, Nat. Commun., 2013, 4, 2642.

158. W. Zhou, X. Zou, S. Najmaei, Z. Liu, Y. Shi, J. Kong, J. Lou, P. M. Ajayan, B. I. Yakobson and J.-C. Idrobo, Nano Lett., 2013, 13, 2615.

159. S. Tongay, J. Suh, C. Ataca, W. Fan, A. Luce, J. S. Kang, J. Liu, C. Ko, R. Raghunathanan, J. Zhou, F. Ogletree, J. Li, J. C. Grossman and J. Wu, Sci. Rep., 2013, 3, 2657.

160. C. P. Lu, G. H. Li, J. H. Mao, L. M. Wang and E. Y. Andrei, Nano Lett., 2014, 14, 4628.

161. S. McDonnell, R. Addou, C. Buie, R. M. Wallace and C. L. Hinkle, ACS Nano, $2014,8,2880$.

162. R. Addou, L. Colombo and R. M. Wallace, ACS. Appl. Mater. Interfaces, 2015, 7, 11921.

163. R. Addou, S. McDonnell, D. Barrera, Z. B. Guo, A. Azcatl, J. Wang, H. Zhu, C. L. Hinkle, M. Quevedo-Lopez, H. N. Alshareef, L. Colombo, J. W. P. Hsu and R. M. Wallace, ACS Nano, 2015, 9, 9124.

164. J. Hong, Z. Hu, M. Probert, K. Li, D. Lv, X. Yang, L. Gu, N. Mao, Q. Feng, L. Xie, J. Zhang, D. Wu, Z. Zhang, C. Jin, W. Ji, X. Zhang, J. Yuan and Z. Zhang, Nat. Commun., $2015,6,6293$.

165. M. Yarali, X. F. Wu, T. Gupta, D. Ghoshal, L. X. Xie, Z. Zhu, H. Brahmi, J. M. Bao, S. Chen, T. F. Luo, N. Koratkar and A. Mavrokefalos, Adv. Funct. Mater., 2017, 27, 1704357.

166. S. Najmaei, J. T. Yuan, J. Zhang, P. Ajayan and J. Lou, Acc. Chem. Res., 2015, 48, 31.

167. P. Vancso, G. Z. Magda, J. Peto, J. Y. Noh, Y. S. Kim, C. Hwang, L. P. Biró and L. Tapaszto, Sci. Rep., 2016, 6, 29726.

168. M. Ghorbani-Asl, A. N. Enyashin, A. Kuc, G. Seifert and T. Heine, Phys. Rev., B, $2013, \mathbf{8 8}, 245440$.

169. D. Liu, Y. Guo, L. Fang and J. Robertson, Appl. Phys. Lett., 2013, 103, 183113.

170. J.-Y. Noh, H. Kim and Y.-S. Kim, Phys. Rev. B, 2014, 89, 205417.

171. K. C. Santosh, R. C. Longo, R. Addou, R. M. Wallace and K. Cho, Nanotechnology, 2014, 25, 375703 .

172. S. J. Yuan, R. Roldan, M. I. Katsnelson and F. Guinea, Phys. Rev., B, 2014, 90, 041402.

173. M. Pizzochero and O. V. Yazyev, Phys. Rev., B, 2017, 96, 245402.

174. H.-P. Komsa and A. V. Krasheninnikov, Phys. Rev., B, 2015, 91, 125304.

175. P. K. Chow, R. B. Jacobs-Gedrim, J. Gao, T. M. Lu, B. Yu, H. Terrones and N. Koratkar, ACS Nano, 2015, 9, 1520.

176. Y. Guo, D. Liu and J. Robertson, Appl. Phys. Lett., 2015, 106, 173106.

177. O. Lehtinen, H. P. Komsa, A. Pulkin, M. B. Whitwick, M. W. Chen, T. Lehnert, M. J. Mohn, O. V. Yazyev, A. Kis, U. Kaiser and A. V. Krasheninnikov, ACS Nano, 2015, 9, 3274 .

178. J. H. Lin, S. T. Pantelides and W. Zhou, ACS Nano, 2015, 9, 5189.

179. M. Pandey, F. A. Rasmussen, K. Kuhar, T. Olsen, K. W. Jacobsen and K. S. Thygesen, Nano Lett., 2016, 16, 2234.

180. M. A. Khan, M. Erementchouk, J. Hendrickson and M. N. Leuenberger, Phys. Rev., $B, 2017,95,245435$.

181. M. Makarova, Y. Okawa and M. Aono, J. Phys. Chem. C, 2012, 116, 22411.

182. Z. Yu, Y. Pan, Y. Shen, Z. Wang, Z.-Y. Ong, T. Xu, R. Xin, L. Pan, B. Wang, L. Sun, J. Wang, G. Zhang, Y. W. Zhang, Y. Shi and X. Wang, Nat. Commun., 2014, 5, 5290. 
183. Q. Li, Y. H. Zhao, C. Y. Ling, S. J. Yuan, Q. Chen and J. L. Wang, Angew. Chem. Int. Ed., 2017, 56, 10501.

184. A. Förster, S. Gemming, G. Seifert and D. Tomanek, ACS Nano, 2017, 11, 9989.

185. H. Li, C. Tsai, A. L. Koh, L. Cai, A. W. Contryman, A. H. Fragapane, J. Zhao, H. S. Han, H. C. Manoharan, F. Abild-Pedersen, J. K. Norskov and X. Zheng, Nat. Mater., 2016, 15,48 .

186. C. Tsai, H. Li, S. Park, J. Park, H. S. Han, J. K. Nørskov, X. Zheng and F. AbildPedersen, Nat. Commun., 2017, 8, 15113.

187. C. B. Roxlo, H. W. Deckman, J. Gland, S. D. Cameron and R. R. Chianelli, Science, 1987, 235, 1629.

188. A. Tuxen, J. Kibsgaard, H. Gobel, E. Laegsgaard, H. Topsoe, J. V. Lauritsen and F. Besenbacher, ACS Nano, 2010, 4, 4677.

189. B. Ni and X. Wang, Advanced Science, 2015, 2, 1500085.

190. J. D. Wiensch, J. John, J. M. Velazquez, D. A. Torelli, A. P. Pieterick, M. T. McDowell, K. Sun, X. Zhao, B. S. Brunschwig and N. S. Lewis, ACS Energy Lett., 2017, 2, 2234.

191. T. F. Jaramillo, K. P. Jorgensen, J. Bonde, J. H. Nielsen, S. Horch and I. Chorkendorff, Science, 2007, 317, 100.

192. Y. Yuan, R. Li and Z. Liu, Anal. Chem., 2014, 86, 3610.

193. T. Wang, R. Zhu, J. Zhuo, Z. Zhu, Y. Shao and M. Li, Anal. Chem., 2014, 86, 12064.

194. M. Jeong, S. Kim and S.-Y. Ju, RSC Adv., 2016, 6, 36248.

195. R. Canton-Vitoria, Y. Sayed-Ahmad-Baraza, M. Pelaez-Fernandez, R. Arenal, C. Bittencourt, C. P. Ewels and N. Tagmatarchis, npj 2D Mater. Appl., 2017, 1, 13.

196. H. Lee, S. Bak, S.-J. An, J. H. Kim, E. Yun, M. Kim, S. Seo, M. S. Jeong and H. Lee, ACS Nano, 2017, 11, 12832.

197. R. C. T. Howe, G. Hu, Z. Yang and T. Hasan, Proc. SPIE, 2015, 9553, 95530R.

198. Z. Li and S. L. Wong, Mat. Sci. Eng., C, 2017, 70, 1095.

199. P. Samorì, V. Palermo and X. L. Feng, Adv. Mater., 2016, 28, 6027.

200. A. Hirsch and F. Hauke, Angew. Chem. Int. Ed., 2018, DOI: 10.1002/anie.201708211.

201. M. Y. Tsai, S. Y. Zhang, P. M. Campbell, R. R. Dasari, X. C. Ba, A. Tarasov, S. Graham, S. Barlow, S. R. Marder and E. M. Vogel, Chem. Mater., 2017, 29, 7296.

202. T. Zhu, L. Yuan, Y. Zhao, M. Zhou, Y. Wan, J. Mei and L. Huang, Sci. Adv., 2018, 4, eaao3104.

203. A. Ciesielski and P. Samorì, Adv. Mater., 2016, 28, 6030.

204. A. Painelli and F. Terenziani, in Non-Linear Optical Properties of Matter: From Molecules to Condensed Phases, eds. M. G. Papadopoulos, A. J. Sadlej and J. Leszczynski, Springer Netherlands, Dordrecht, 2006, ch. 7, pp. 251.

205. B. Chamlagain, Q. Li, N. J. Ghimire, H. J. Chuang, M. M. Perera, H. Tu, Y. Xu, M. Pan, D. Xaio, J. Yan, D. Mandrus and Z. Zhou, ACS Nano, 2014, 8, 5079.

206. B. Liu, W. Zhao, Z. Ding, I. Verzhbitskiy, L. Li, J. Lu, J. Chen, G. Eda and K. P. Loh, Adv. Mater., 2016, 28, 6457.

207. D. Voiry, A. Mohite and M. Chhowalla, Chem. Soc. Rev., 2015, 44, 2702.

208. Y. J. Gong, Z. Liu, A. R. Lupini, G. Shi, J. H. Lin, S. Najmaei, Z. Lin, A. L. Elias, A. Berkdemir, G. You, H. Terrones, M. Terrones, R. Vajtai, S. T. Pantelides, S. J. Pennycook, J. Lou, W. Zhou and P. M. Ajayan, Nano Lett., 2014, 14, 442.

209. $\quad$ S. Mouri, Y. Miyauchi and K. Matsuda, Nano Lett., 2013, 13, 5944.

210. C. Gong, H. J. Zhang, W. H. Wang, L. Colombo, R. M. Wallace and K. J. Cho, Appl. Phys. Lett., 2013, 103, 053513.

211. K. Keyshar, M. Berg, X. Zhang, R. Vajtai, G. Gupta, C. K. Chan, T. E. Beechem, P. M. Ajayan, A. D. Mohite and T. Ohta, ACS Nano, 2017, 11, 8223.

212. S. L. Howell, D. Jariwala, C. C. Wu, K. S. Chen, V. K. Sangwan, J. M. Kang, T. J. Marks, M. C. Hersam and L. J. Lauhon, Nano Lett., 2015, 15, 2278.

213. S.-H. Jo, D.-H. Kang, J. Shim, J. Jeon, M. H. Jeon, G. Yoo, J. Kim, J. Lee, G. Y. Yeom, S. Lee, H.-Y. Yu, C. Choi and J.-H. Park, Adv. Mater., 2016, 28, 4824.

214. I. Lee, S. Rathi, L. Li, D. Lim, M. A. Khan, E. S. Kannan and G.-H. Kim, Nanotechnology, 2015, 26, 455203. 
215. L. Yu, A. Zubair, E. J. G. Santos, X. Zhang, Y. Lin, Y. Zhang and T. Palacios, Nano Lett., 2015, 15, 4928.

216. N. Peimyoo, W. H. Yang, J. Z. Shang, X. N. Shen, Y. L. Wang and T. Yu, ACS Nano, 2014, 8, 11320.

217. J. H. Park, A. Sanne, Y. Z. Guo, M. Amani, K. H. Zhang, H. C. P. Movva, J. A. Robinson, A. Javey, J. Robertson, S. K. Banerjee and A. C. Kummel, Sci. Adv., 2017, 3, e1701661.

218. A. J. Molina-Mendoza, L. Vaquero-Garzon, S. Leret, L. de Juan-Fernandez, E. M. Perez and A. Castellanos-Gomez, Chem. Commun., 2016, 52, 14365.

219. Z. Song, T. Schultz, Z. Ding, B. Lei, C. Han, P. Amsalem, T. Lin, D. Chi, S. L. Wong, Y. J. Zheng, M.-Y. Li, L.-J. Li, W. Chen, N. Koch, Y. L. Huang and A. T. S. Wee, ACS Nano, 2017, 11, 9128.

220. H. Y. Park, S. R. Dugasani, D. H. Kang, G. Yoo, J. Kim, B. Gnapareddy, J. Jeon, M. Kim, Y. J. Song, S. Lee, J. Heo, Y. J. Jeon, S. H. Park and J. H. Park, Sci. Rep., 2016, 6, 35733.

221. D. H. Kang, S. R. Dugasani, H. Y. Park, J. Shim, B. Gnapareddy, J. Jeon, S. Lee, Y. Roh, S. H. Park and J. H. Park, Sci. Rep., 2016, 6, 20333.

222. J. Li, J. Wierzbowski, Ö. Ceylan, J. Klein, F. Nisic, T. Le Anh, F. Meggendorfer, C. A. Palma, C. Dragonetti, J. V. Barth, J. J. Finley and E. Margapoti, Appl. Phys. Lett., 2014, 105, 241116.

223. M. Mahjouri-Samani, L. Liang, A. Oyedele, Y.-S. Kim, M. Tian, N. Cross, K. Wang, M.-W. Lin, A. Boulesbaa, C. M. Rouleau, A. A. Puretzky, K. Xiao, M. Yoon, G. Eres, G. Duscher, B. G. Sumpter and D. B. Geohegan, Nano Lett., 2016, 16, 5213.

224. N. Ma and D. Jena, Phys. Rev. X, 2014, 4, 011043.

225. A. Di Bartolomeo, L. Genovese, T. Foller, F. Giubileo, G. Luongo, L. Croin, S. J. Liang, L. K. Ang and M. Schleberger, Nanotechnology, 2017, 28, 214002.

226. K. F. Mak, K. L. He, C. Lee, G. H. Lee, J. Hone, T. F. Heinz and J. Shan, Nat. Mater., 2013, 12, 207.

227. B. Chakraborty, A. Bera, D. V. S. Muthu, S. Bhowmick, U. V. Waghmare and A. K. Sood, Phys. Rev., B, 2012, 85, 161403.

228. R. Saito, Y. Tatsumi, S. Huang, X. Ling and M. S. Dresselhaus, J. Phys.: Condens. Matter, 2016, 28, 353002.

229. C. Wirtz, T. Hallam, C. P. Cullen, N. C. Berner, M. O'Brien, M. Marcia, A. Hirsch and G. S. Duesberg, Chem. Commun., 2015, 51, 16553.

230. O. A. Ajayi, J. V. Ardelean, G. D. Shepard, J. Wang, A. Antony, T. Taniguchi, K. Watanabe, T. F. Heinz, S. Strauf, X. Y. Zhu and J. C. Hone, 2D Mater., 2017, 4, 0310011.

231. S. De Feyter and F. C. De Schryver, Chem. Soc. Rev., 2003, 32, 139.

232. C.-A. Palma and P. Samorì, Nat. Chem., 2011, 3, 431.

233. A. Ciesielski, C.-A. Palma, M. Bonini and P. Samorì, Adv. Mater., 2010, 22, 3506.

234. C.-A. Palma, M. Cecchini and P. Samori, Chem. Soc. Rev., 2012, 41, 3713.

235. V. Podzorov, M. E. Gershenson, C. Kloc, R. Zeis and E. Bucher, Appl. Phys. Lett., 2004, 84, 3301.

236. W. Bao, X. Cai, D. Kim, K. Sridhara and M. S. Fuhrer, Appl. Phys. Lett., 2013, 102, 042104.

237. M. Buscema, G. A. Steele, H. S. J. van der Zant and A. Castellanos-Gomez, Nano Res., 2014, 7, 1.

238. D. Akinwande, N. Petrone and J. Hone, Nat. Commun., 2014, 5, 5678.

239. J. Pu, Y. Yomogida, K.-K. Liu, L.-J. Li, Y. Iwasa and T. Takenobu, Nano Lett., 2012, 12, 4013.

240. H. Sirringhaus, Adv. Mater., 2005, 17, 2411.

241. I. N. Hulea, S. Fratini, H. Xie, C. L. Mulder, N. N. Iossad, G. Rastelli, S. Ciuchi and A. F. Morpurgo, Nat. Mater., 2006, 5, 982.

242. J. Tsibouklis and T. G. Nevell, Adv. Mater., 2003, 15, 647.

243. K. Dolui, I. Rungger and S. Sanvito, Phys. Rev., B, 2013, 87, 165402.

244. J. C. Love, L. A. Estroff, J. K. Kriebel, R. G. Nuzzo and G. M. Whitesides, Chem. Rev., 2005, 105, 1103.

245. F. Schreiber, Prog. Surf. Sci., 2000, 65, 151. 
247. E. Margapoti, J. Li, Ö. Ceylan, M. Seifert, F. Nisic, T. L. Anh, F. Meggendorfer, C. Dragonetti, C.-A. Palma, J. V. Barth and J. J. Finley, Adv. Mater., 2015, 27, 1426.

248. H. M. D. Bandara and S. C. Burdette, Chem. Soc. Rev., 2012, 41, 1809.

249. N. Crivillers, A. Liscio, F. Di Stasio, C. Van Dyck, S. Osella, D. Cornil, S. Mian, G. M. Lazzerini, O. Fenwick, E. Orgiu, F. Reinders, S. Braun, M. Fahlman, M. Mayor, J. Cornil, V. Palermo, F. Cacialli and P. Samorì, Phys. Chem. Chem. Phys., 2011, 13, 14302.

250. G. Pace, V. Ferri, C. Grave, M. Elbing, C. von Hänisch, M. Zharnikov, M. Mayor, M. A. Rampi and P. Samorì, Proc. Natl. Acad. Sci. USA, 2007, 104, 9937.

251. N. Crivillers, S. Osella, C. Van Dyck, G. M. Lazzerini, D. Cornil, A. Liscio, F. Di Stasio, S. Mian, O. Fenwick, F. Reinders, M. Neuburger, E. Treossi, M. Mayor, V. Palermo, F. Cacialli, J. Cornil and P. Samorì, Adv. Mater., 2013, 25, 432.

252. J. Wang, H. Yu, X. Zhou, X. Liu, R. Zhang, Z. Lu, J. Zheng, L. Gu, K. Liu, D. Wang and L. Jiao, Nat. Commun., 2017, 8, 377.

253. S. Cincotti and J. P. Rabe, Appl. Phys. Lett., 1993, 62, 3531.

254. L. Cheng, J. Lee, H. Zhu, A. V. Ravichandran, Q. Wang, A. T. Lucero, M. J. Kim, R. M. Wallace, L. Colombo and J. Kim, ACS Nano, 2017, 11, 10243.

255. K. P. Dhakal, D. L. Duong, J. Lee, H. Nam, M. Kim, M. Kan, Y. H. Lee and J. Kim, Nanoscale, 2014, 6, 13028.

256. S. Tongay, K. Berke, M. Lemaitre, Z. Nasrollahi, D. B. Tanner, A. F. Hebard and B. R. Appleton, Nanotechnology, 2011, 22, 425701.

257. H. Kim, D. H. Lien, M. Amani, J. W. Ager and A. Javey, ACS Nano, 2017, 11, 5179.

258. X. Zhang, Q. Liao, S. Liu, Z. Kang, Z. Zhang, J. Du, F. Li, S. Zhang, J. Xiao, B. Liu, Y. Ou, X. Liu, L. Gu and Y. Zhang, Nat. Commun., 2017, 8, 1.

259. S. S. Chou, M. De, J. Kim, S. Byun, C. Dykstra, J. Yu, J. Huang and V. P. Dravid, J. Am. Chem. Soc., 2013, 135, 4584.

260. K. C. Knirsch, N. C. Berner, H. C. Nerl, C. S. Cucinotta, Z. Gholamvand, N. McEvoy, Z. Wang, I. Abramovic, P. Vecera, M. Halik, S. Sanvito, G. S. Duesberg, V. Nicolosi, F. Hauke, A. Hirsch, J. N. Coleman and C. Backes, ACS Nano, 2015, 9, 6018.

261. X. Chen, N. C. Berner, C. Backes, G. S. Duesberg and A. R. McDonald, Angew. Chem. Int. Ed., 2016, 55, 5803.

262. A. Azcatl, S. McDonnell, K. C. Santosh, X. Peng, H. Dong, X. Y. Qin, R. Addou, G. I. Mordi, N. Lu, J. Kim, M. J. Kim, K. Cho and R. M. Wallace, Appl. Phys. Lett., 2014, 104, 111601 .

263. L. X. Cheng, X. Y. Qin, A. T. Lucero, A. Azcatl, J. Huang, R. M. Wallace, K. Cho and J. Kim, ACS. Appl. Mater. Interfaces, 2014, 6, 11834.

264. H. P. Komsa, S. Kurasch, O. Lehtinen, U. Kaiser and A. V. Krasheninnikov, Phys. Rev., $B, 2013, \mathbf{8 8}, 5143$.

265. W. M. Parkin, A. Balan, L. Liang, P. M. Das, M. Lamparski, C. H. Naylor, J. A. Rodríguez-Manzo, A. T. C. Johnson, V. Meunier and M. Drndić, ACS Nano, 2016, 10, 4134.

266. E. Sutter, Y. Huang, H. P. Komsa, M. Ghorbani-Asl, A. V. Krasheninnikov and P. Sutter, Nano Lett., 2016, 16, 4410.

267. D. Karmakar, R. Halder, N. Padma, G. Abraham, K. Vaibhav, M. Ghosh, M. Kaur, D. Bhattacharya and T. V. C. Rao, J. Appl. Phys., 2015, 117, 135701.

268. M. Donarelli, F. Bisti, F. Perrozzi and L. Ottaviano, Chem. Phys. Lett., 2013, 588,

198.

269. H. Y. Nan, Z. L. Wang, W. H. Wang, Z. Liang, Y. Lu, Q. Chen, D. W. He, P. H. Tan, F. Miao, X. R. Wang, J. L. Wang and Z. H. Ni, ACS Nano, 2014, 8, 5738.

270. M. R. Islam, N. Kang, U. Bhanu, H. P. Paudel, M. Erementchouk, L. Tetard, M. N. Leuenberger and S. I. Khondaker, Nanoscale, 2014, 6, 10033.

271. M. Tosun, L. Chan, M. Amani, T. Roy, G. H. Ahn, P. Taheri, C. Carraro, J. W. Ager, R. Maboudian and A. Javey, ACS Nano, 2016, 10, 6853.

272. N. Kang, H. P. Paudel, M. N. Leuenberger, L. Tetard and S. I. Khondaker, J. Phys. Chem. C, 2014, 118, 21258.

273. J. Zhu, Z. Wang, H. Yu, N. Li, J. Zhang, J. Meng, M. Liao, J. Zhao, X. Lu, L. Du, R. Yang, D. Shi, Y. Jiang and G. Zhang, J. Am. Chem. Soc., 2017, 139, 10216. 
274. Q. Ma, P. M. Odenthal, J. Mann, D. Le, C. S. Wang, Y. Zhu, T. Chen, S. Dezheng, K. Yamaguchi, T. Tran, M. Wurch, J. L. McKinley, J. Wyrick, K. Magnone, T. F. Heinz, T. S. Rahman, R. Kawakami and L. Bartels, J. Phys. Condens. Matter, 2013, 25, 252201.

275. L. Ma, Y. Tan, M. Ghorbani-Asl, R. Boettger, S. Kretschmer, S. Zhou, Z. Huang, A. V. Krasheninnikov and F. Chen, Nanoscale, 2017, 9, 11027.

276. D. Kim, H. Du, T. Kim, S. Shin, S. Kim, M. Song, C. Lee, J. Lee, H. Cheong, D. H. Seo and S. Seo, $A I P A d v ., 2016,6,105307$.

277. J. Klein, A. Kuc, A. Nolinder, M. Altzschner, J. Wierzbowski, F. Sigger, F. Kreupl, J. J. Finley, U. Wurstbauer, A. W. Holleitner and M. Kaniber, 2D Mater., 2018, 5, 011007.

278. V. Iberi, L. Liang, A. V. Ievlev, M. G. Stanford, M.-W. Lin, X. Li, M. MahjouriSamani, S. Jesse, B. G. Sumpter, S. V. Kalinin, D. C. Joy, K. Xiao, A. Belianinov and O. S. Ovchinnikova, Sci. Rep., 2016, 6, 30481.

279. D. S. Fox, Y. Zhou, P. Maguire, A. O’Neill, C. Ó’Coileáin, R. Gatensby, A. M. Glushenkov, T. Tao, G. S. Duesberg, I. V. Shvets, M. Abid, M. Abid, H.-C. Wu, Y. Chen, J. N. Coleman, J. F. Donegan and H. Zhang, Nano Lett., 2015, 15, 5307.

280. S. Mignuzzi, A. J. Pollard, N. Bonini, B. Brennan, I. S. Gilmore, M. A. Pimenta, D. Richards and D. Roy, Phys. Rev. B, 2015, 91, 195411.

281. L. Madauss, O. Ochedowski, H. Lebius, B. Ban-d'Etat, C. H. Naylor, A. T. C. Johnson, J. Kotakoski and M. Schleberger, 2D Mater., 2017, 4, 015034.

282. L. Cai, J. He, Q. Liu, T. Yao, L. Chen, W. Yan, F. Hu, Y. Jiang, Y. Zhao, T. Hu, Z. Sun and S. Wei, J. Am. Chem. Soc., 2015, 137, 2622.

283. M. Ghorbani-Asl, S. Kretschmer, D. E. Spearot and A. V. Krasheninnikov, 2D Mater., 2017, 4, 025078.

284. Q. Yue, S. Chang, S. Qin and J. Li, Phys. Lett. A, 2013, 377, 1362.

285. K. Dolui, I. Rungger, C. Das Pemmaraju and S. Sanvito, Phys. Rev., B, 2013, 88, 075420.

286. R. H. Williams, I. G. Higginbotham and M. A. B. Whitaker, J. Phys. C: Solid State Phys., 1972, 5, L191.

287. H. C. Feng and J. M. Chen, J. Phys. C: Solid State Phys., 1974, 7, L75.

288. N. S. Mcintyre, P. A. Spevack, G. Beamson and D. Briggs, Surf. Sci., 1990, 237, L390.

289. S. Naohisa and O. Keiichi, Jpn. J. Appl. Phys., 1995, 34, 3363.

290. M. A. Baker, R. Gilmore, C. Lenardi and W. Gissler, Appl. Surf. Sci., 1999, 150,

255.

291. J. B. Park, C. B. France and B. A. Parkinson, J. Vac. Sci. Technol. B, 2005, 23, 1532.

292. S. M. Davis and J. C. Carver, Appl. Surf. Sci., 1984, 20, 193.

293. K. Cho, M. Min, T.-Y. Kim, H. Jeong, J. Pak, J.-K. Kim, J. Jang, S. J. Yun, Y. H. Lee, W.-K. Hong and T. Lee, ACS Nano, 2015, 9, 8044.

294. A. Aoshima and H. Wise, J. Catal., 1974, 34, 145.

295. S. L. Peterson and K. H. Schulz, Langmuir, 1996, 12, 941.

296. C. G. Wiegenstein and K. H. Schulz, J. Phys. Chem. B, 1999, 103, 6913.

297. S.-L. Li, K. Tsukagoshi, E. Orgiu and P. Samorì, Chem. Soc. Rev., 2016, 45, 118.

298. H. Qiu, L. J. Pan, Z. N. Yao, J. J. Li, Y. Shi and X. R. Wang, Appl. Phys. Lett., 2012, 100, 123104.

299. R. G. Nuzzo, B. R. Zegarski and L. H. Dubois, J. Am. Chem. Soc., 1987, 109, 733.

300. D. G. Castner, K. Hinds and D. W. Grainger, Langmuir, 1996, 12, 5083.

301. H. Gronbeck, A. Curioni and W. Andreoni, J. Am. Chem. Soc., 2000, 122, 3839.

302. L. Kankate, A. Turchanin and A. Gölzhäuser, Langmuir, 2009, 25, 10435.

303. Y. R. Xue, X. Li, H. B. Li and W. K. Zhang, Nat. Commun., 2014, 5, 4348.

304. J. P. Lu, A. Carvalho, X. K. Chan, H. W. Liu, B. Liu, E. S. Tok, K. P. Loh, A. H. C. Neto and C. H. Sow, Nano Lett., 2015, 15, 3524.

305. S. Flemer, Molecules, 2011, 16, 3232.

306. X. Wu, H. Li, H. Liu, X. Zhuang, X. Wang, X. Fan, X. Duan, X. Zhu, Q. Zhang, A. J. Meixner, X. Duan and A. Pan, Nanoscale, 2017, 9, 4707.

307. M. Naz, T. Hallam, N. C. Berner, N. McEvoy, R. Gatensby, J. B. McManus, Z. Akhter and G. S. Duesberg, ACS. Appl. Mater. Interfaces, 2016, 8, 31442.

308. R. Kappera, D. Voiry, S. E. Yalcin, B. Branch, G. Gupta, A. D. Mohite and M. Chhowalla, Nat. Mater., 2014, 13, 1128. 
309. M. B. Dines, J. Chem. Educ., 1974, 51, 221.

310. E. Benavente, M. A. Santa Ana, F. Mendizábal and G. González, Coord. Chem. Rev., 2002, 224, 87.

311. Y. Yin, J. Han, Y. Zhang, X. Zhang, P. Xu, Q. Yuan, L. Samad, X. Wang, Y. Wang, Z. Zhang, P. Zhang, X. Cao, B. Song and S. Jin, J. Am. Chem. Soc., 2016, 138, 7965.

312. X. Fan, P. Xu, Y. C. Li, D. Zhou, Y. Sun, M. A. T. Nguyen, M. Terrones and T. E. Mallouk, J. Am. Chem. Soc., 2016, 138, 5143.

313. L. Niu, J. N. Coleman, H. Zhang, H. Shin, M. Chhowalla and Z. Zheng, Small, 2016, $12,272$.

314. J. I. Paredes and S. Villar-Rodil, Nanoscale, 2016, 8, 15389.

315. X. Zhang, Z. Lai, C. Tan and H. Zhang, Angew. Chem. Int. Ed., 2016, 55, 8816.

316. E. D. Grayfer, M. N. Kozlova and V. E. Fedorov, Adv. Colloid Interface Sci., 2017, 245, 40 .

317. H. Tao, Y. Zhang, Y. Gao, Z. Sun, C. Yan and J. Texter, Phys. Chem. Chem. Phys., 2017, 19, 921 .

318. M. Gutierrez and A. Henglein, Ultrasonics, 1989, 27, 259.

319. R. J. Smith, P. J. King, M. Lotya, C. Wirtz, U. Khan, S. De, A. O'Neill, G. S. Duesberg, J. C. Grunlan, G. Moriarty, J. Chen, J. Wang, A. I. Minett, V. Nicolosi and J. N. Coleman, Adv. Mater., 2011, 23, 3944.

320. J. Kang, V. K. Sangwan, J. D. Wood and M. C. Hersam, Acc. Chem. Res., 2017, 50,

943.

321. C. Tan and H. Zhang, Chem. Soc. Rev., 2015, 44, 2713.

322. X. Chen, R. A. Boulos, P. K. Eggers and C. L. Raston, Chem. Commun., 2012, 48, 11407.

323. Y. Yao, Z. Lin, Z. Li, X. Song, K.-S. Moon and C.-p. Wong, J. Mater. Chem., 2012, 22, 13494.

324. C. Backes, R. J. Smith, N. McEvoy, N. C. Berner, D. McCloskey, H. C. Nerl, A. O’Neill, P. J. King, T. Higgins, D. Hanlon, N. Scheuschner, J. Maultzsch, L. Houben, G. S. Duesberg, J. F. Donegan, V. Nicolosi and J. N. Coleman, Nat. Commun., 2014, 5, 4576.

325. X. Yu, M. S. Prévot and K. Sivula, Chem. Mater., 2014, 26, 5892.

326. A. Gupta, V. Arunachalam and S. Vasudevan, J. Phys. Chem. Lett., 2015, 6, 739.

327. M. Zhang, R. C. T. Howe, R. I. Woodward, E. J. R. Kelleher, F. Torrisi, G. Hu, S. V. Popov, J. R. Taylor and T. Hasan, Nano Res., 2015, 8, 1522.

328. C. Backes, B. M. Szydłowska, A. Harvey, S. Yuan, V. Vega-Mayoral, B. R. Davies, P.-1. Zhao, D. Hanlon, E. J. G. Santos, M. I. Katsnelson, W. J. Blau, C. Gadermaier and J. N. Coleman, ACS Nano, 2016, 10, 1589.

329. G. Liu and N. Komatsu, ChemNanoMat, 2016, 2, 500.

330. G. Liu and N. Komatsu, ChemPhysChem, 2016, 17, 1557.

331. D. Mao, B. Du, D. Yang, S. Zhang, Y. Wang, W. Zhang, X. She, H. Cheng, H. Zeng and J. Zhao, Small, 2016, 12, 1489.

332. L. Guardia, J. I. Paredes, R. Rozada, S. Villar-Rodil, A. Martinez-Alonso and J. M. D. Tascon, $R S C A d v$., 2014, 4, 14115.

333. J. Liu, Z. Zeng, X. Cao, G. Lu, L.-H. Wang, Q.-L. Fan, W. Huang and H. Zhang, Small, 2012, 8, 3517.

334. P. May, U. Khan, J. M. Hughes and J. N. Coleman, J. Phys. Chem. C, 2012, 116, 11393.

335. M. D. J. Quinn, N. H. Ho and S. M. Notley, ACS. Appl. Mater. Interfaces, 2013, 5, 12751.

336. P. Joo, K. Jo, G. Ahn, D. Voiry, H. Y. Jeong, S. Ryu, M. Chhowalla and B.-S. Kim, Nano Lett., 2014, 14, 6456.

337. Y. Kang, S. Najmaei, Z. Liu, Y. Bao, Y. Wang, X. Zhu, N. J. Halas, P. Nordlander, P. M. Ajayan, J. Lou and Z. Fang, Adv. Mater., 2014, 26, 6467.

338. R. Bari, D. Parviz, F. Khabaz, C. D. Klaassen, S. D. Metzler, M. J. Hansen, R. Khare and M. J. Green, Phys. Chem. Chem. Phys., 2015, 17, 9383.

339. M.-K. Chuang, S.-S. Yang and F.-C. Chen, Materials, 2015, 8, 5252.

340. L. David, R. Bhandavat, U. Barrera and G. Singh, Sci. Rep., 2015, 5, 9792.

341. N. D. Mansukhani, L. M. Guiney, P. J. Kim, Y. Zhao, D. Alducin, A. Ponce, E. Larios, M. J. Yacaman and M. C. Hersam, Small, 2016, 12, 294. 
342. R. C. Selhorst, E. Puodziukynaite, J. A. Dewey, P. Wang, M. D. Barnes, A. Ramasubramaniam and T. Emrick, Chem. Sci., 2016, 7, 4698.

343. V. Vega-Mayoral, C. Backes, D. Hanlon, U. Khan, Z. Gholamvand, M. O'Brien, G. S. Duesberg, C. Gadermaier and J. N. Coleman, Adv. Funct. Mater., 2016, 26, 1028.

344. X. Zhao, X. Ma, J. Sun, D. Li and X. Yang, ACS Nano, 2016, 10, 2159.

345. S. M. Notley, J. Colloid Interface Sci., 2013, 396, 160.

346. Y. Ge, J. Wang, Z. Shi and J. Yin, J. Mater. Chem., 2012, 22, 17619.

347. F. Zhang, X. Chen, R. A. Boulos, F. Md Yasin, H. Lu, C. Raston and H. Zhang, Chem. Commun., 2013, 49, 4845.

348. X. Feng, X. Wang, W. Xing, K. Zhou, L. Song and Y. Hu, Compos. Sci. Technol., 2014, 93, 76 .

349. B. L. Li, H. Q. Luo, J. L. Lei and N. B. Li, RSC Adv., 2014, 4, 24256.

350. W. Yin, L. Yan, J. Yu, G. Tian, L. Zhou, X. Zheng, X. Zhang, Y. Yong, J. Li, Z. Gu and Y. Zhao, ACS Nano, 2014, 8, 6922.

351. Y. Yong, L. Zhou, Z. Gu, L. Yan, G. Tian, X. Zheng, X. Liu, X. Zhang, J. Shi, W. Cong, W. Yin and Y. Zhao, Nanoscale, 2014, 6, 10394.

352. G. Guan, S. Zhang, S. Liu, Y. Cai, M. Low, C. P. Teng, I. Y. Phang, Y. Cheng, K. L. Duei, B. M. Srinivasan, Y. Zheng, Y.-W. Zhang and M.-Y. Han, J. Am. Chem. Soc., 2015, $137,6152$.

353. R.-M. Kong, L. Ding, Z. Wang, J. You and F. Qu, Anal. Bioanal.Chem., 2015, 407, 369.

354. B. L. Li, H. L. Zou, L. Lu, Y. Yang, J. L. Lei, H. Q. Luo and N. B. Li, Adv. Funct. Mater., 2015, 25, 3541.

355. Y. Li, H. Zhu, F. Shen, J. Wan, S. Lacey, Z. Fang, H. Dai and L. Hu, Nano Energy, $2015, \mathbf{1 3}, 346$.

356. W. Liu, C. Zhao, R. Zhou, D. Zhou, Z. Liu and X. Lu, Nanoscale, 2015, 7, 9919.

357. S. Ravula, J. B. Essner and G. A. Baker, ChemNanoMat, 2015, 1, 167.

358. D. Wang, L. Song, K. Zhou, X. Yu, Y. Hu and J. Wang, J. Mater. Chem. A, 2015, 3, 14307.

359. W. Zhang, Y. Wang, D. Zhang, S. Yu, W. Zhu, J. Wang, F. Zheng, S. Wang and J. Wang, Nanoscale, 2015, 7, 10210.

360. G. S. Bang, S. Cho, N. Son, G. W. Shim, B.-K. Cho and S.-Y. Choi, ACS. Appl. Mater. Interfaces, $2016, \mathbf{8}, 1943$.

361. N. Kapil, A. Singh, M. Singh and D. Das, Angew. Chem. Int. Ed., 2016, 55, 7772.

362. A. Ciesielski and P. Samori, Chem. Soc. Rev., 2014, 43, 381.

363. S. Lin, C.-J. Shih, M. S. Strano and D. Blankschtein, J. Am. Chem. Soc., 2011, 133, 12810 .

364. Z. Gholamvand, D. McAteer, C. Backes, N. McEvoy, A. Harvey, N. C. Berner, D. Hanlon, C. Bradley, I. Godwin, A. Rovetta, M. E. G. Lyons, G. S. Duesberg and J. N. Coleman, Nanoscale, 2016, 8, 5737.

365. D. McAteer, Z. Gholamvand, N. McEvoy, A. Harvey, E. O’Malley, G. S. Duesberg and J. N. Coleman, ACS Nano, 2016, 10, 672.

366. G. B. de-Mello, L. Smith, S. J. Rowley-Neale, J. Gruber, S. J. Hutton and C. E. Banks, RSC Adv., 2017, 7, 36208.

367. J. Kang, J.-W. T. Seo, D. Alducin, A. Ponce, M. J. Yacaman and M. C. Hersam, Nat. Commun., 2014, 5, 5478.

368. Y. Hu, Y. Huang, C. Tan, X. Zhang, Q. Lu, M. Sindoro, X. Huang, W. Huang, L. Wang and H. Zhang, Mater. Chem. Front., 2017, 1, 24.

369. W. Liu, X. Yang, Y. Zhang, M. Xu and H. Chen, RSC Adv., 2014, 4, 32744.

370. K. Q. Zhou, J. J. Liu, P. Y. Wen, Y. Hu and Z. Gui, Appl. Surf. Sci., 2014, 316, 237.

371. S. S. Chou, Y.-K. Huang, J. Kim, B. Kaehr, B. M. Foley, P. Lu, C. Dykstra, P. E. Hopkins, C. J. Brinker, J. Huang and V. P. Dravid, J. Am. Chem. Soc., 2015, 137, 1742.

372. J. I. Paredes, J. M. Munuera, S. Villar-Rodil, L. Guardia, M. Ayan-Varela, A. Pagan, S. D. Aznar-Cervantes, J. L. Cenis, A. Martinez-Alonso and J. M. D. Tascon, ACS. Appl. Mater. Interfaces, 2016, 8, 27974.

373. M. Cai, F. Zhang, C. Zhang, C. Lu, Y. He, Y. Qu, H. Tian, X. Feng and X. Zhuang, J. Mater. Chem. A, 2018, 6, 138. 
374. X. Chen, D. McAteer, C. McGuinness, I. Godwin, J. N. Coleman and A. R. McDonald, Chem. Eur. J., 2018, 24, 351.

375. J. M. Englert, C. Dotzer, G. Yang, M. Schmid, C. Papp, J. M. Gottfried, H.-P. Steinrück, E. Spiecker, F. Hauke and A. Hirsch, Nat. Chem., 2011, 3, 279.

376. Y.-T. Liu, Z. Tan, X.-M. Xie, Z.-F. Wang and X.-Y. Ye, Chem-Asian J., 2013, 8,

817.

377. M. N. Tahir, N. Zink, M. Eberhardt, H. A. Therese, U. Kolb, P. Theato and W. Tremel, Angew. Chem. Int. Ed., 2006, 45, 4809.

378. S. Lei, X. Wang, B. Li, J. Kang, Y. He, A. George, L. Ge, Y. Gong, P. Dong, Z. Jin, G. Brunetto, W. Chen, Z.-T. Lin, R. Baines, D. S. Galvão, J. Lou, E. Barrera, K. Banerjee, R. Vajtai and P. Ajayan, Nat. Nanotechnol., 2016, 11, 465.

379. R. Anbazhagan, H.-J. Wang, H.-C. Tsai and R.-J. Jeng, RSC Adv., 2014, 4, 42936.

380. S.-D. Jiang, G. Tang, Z.-M. Bai, Y.-Y. Wang, Y. Hu and L. Song, RSC Adv., 2014, 4, 3253 .

381. J.-S. Kim, H.-W. Yoo, H. O. Choi and H.-T. Jung, Nano Lett., 2014, 14, 5941.

382. Z. Tang, Q. Wei and B. Guo, Chem. Commun., 2014, 50, 3934.

383. L. Zhou, B. He, Y. Yang and Y. He, RSC Adv., 2014, 4, 32570.

384. T. Liu, S. Shi, C. Liang, S. Shen, L. Cheng, C. Wang, X. Song, S. Goel, T. E. Barnhart, W. Cai and Z. Liu, ACS Nano, 2015, 9, 950.

385. S. Pandit, S. Karunakaran, S. K. Boda, B. Basu and M. De, ACS. Appl. Mater. Interfaces, $2016, \mathbf{8}, 31567$.

386. X. Yang, N. Meng, Y. Zhu, Y. Zhou, W. Nie and P. Chen, Journal of Materials Science, 2016, 51, 1344.

387. S. G. McAdams, E. A. Lewis, J. R. Brent, S. J. Haigh, A. G. Thomas, P. O'Brien, F. Tuna and D. J. Lewis, Adv. Funct. Mater., 2017, 27, 1703646.

388. S. Presolski, L. Wang, A. H. Loo, A. Ambrosi, P. Lazar, V. Ranc, M. Otyepka, R. Zboril, O. Tomanec, J. Ugolotti, Z. Sofer and M. Pumera, Chem. Mater., 2017, 29, 2066.

389. L. Cheng, J. Liu, X. Gu, H. Gong, X. Shi, T. Liu, C. Wang, X. Wang, G. Liu, H. Xing, W. Bu, B. Sun and Z. Liu, Adv. Mater., 2014, 26, 1886.

390. Q. Ding, K. J. Czech, Y. Zhao, J. Zhai, R. J. Hamers, J. C. Wright and S. Jin, ACS. Appl. Mater. Interfaces, 2017, 9, 12734.

391. R. H. Gonçalves, R. Fiel, M. R. S. Soares, W. H. Schreiner, C. M. P. Silva and E. R. Leite, Chem. Eur. J., 2015, 21, 15583.

392. H. Liu, N. Han and J. Zhao, RSC Adv., 2015, 5, 17572.

393. J. Martincová, M. Otyepka and P. Lazar, Chem. Eur. J., 2017, 23, 13233.

394. X. Huang, Z. Zeng, S. Bao, M. Wang, X. Qi, Z. Fan and H. Zhang, Nat. Commun., 2013, 4, 1444.

395. J. Kim, S. Byun, A. J. Smith, J. Yu and J. Huang, J. Phys. Chem. Lett., 2013, 4, 1227.

396. Y.-M. Liu, M. Zhou, Y.-Y. Liu, G.-F. Shi, J.-J. Zhang, J.-T. Cao, K.-J. Huang and Y.-H. Chen, RSC Adv., 2014, 4, 22888.

397. X. Xia, Z. Zheng, Y. Zhang, X. Zhao and C. Wang, Sens. Actuators, B, 2014, 192, 42.

398. Z. Yin, B. Chen, M. Bosman, X. Cao, J. Chen, B. Zheng and H. Zhang, Small, 2014, $\mathbf{1 0}, 3537$.

399. L. Yuwen, F. Xu, B. Xue, Z. Luo, Q. Zhang, B. Bao, S. Su, L. Weng, W. Huang and L. Wang, Nanoscale, 2014, 6, 5762.

400. Z. Cheng, B. He and L. Zhou, J. Mater. Chem. A, 2015, 3, 1042.

401. M. A. Hussain, M. Yang, T. J. Lee, J. W. Kim and B. G. Choi, J. Colloid Interface Sci., 2015, 451, 216.

402. S. Su, C. Zhang, L. Yuwen, X. Liu, L. Wang, C. Fan and L. Wang, Nanoscale, 2016, 8, 602 .

403. F. Raza, D. Yim, J. H. Park, H.-I. Kim, S.-J. Jeon and J.-H. Kim, J. Am. Chem. Soc., 2017, 139, 14767.

404. J. R. Dunklin, P. Lafargue, T. M. Higgins, G. T. Forcherio, M. Benamara, N. McEvoy, D. K. Roper, J. N. Coleman, Y. Vaynzof and C. Backes, npj 2D Mater. Appl., 2018, $1,43$.

405. J. Lin, H. Li, H. Zhang and W. Chen, Appl. Phys. Lett., 2013, 102, 203109. 
406. J. Li, Q. Ji, S. Chu, Y. Zhang, Y. Li, Q. Gong, K. Liu and K. Shi, Sci. Rep., 2016, 6, 23626.

407. Z. Li, S. Jiang, Y. Huo, M. Liu, C. Yang, C. Zhang, X. Liu, Y. Sheng, C. Li and B. Man, Opt. Express, 2016, 24, 26097.

408. D. Liu, L. Yu, X. Xiong, L. Yang, Y. Li, M. Li, H.-O. Li, G. Cao, M. Xiao, B. Xiang, C.-j. Min, G.-C. Guo, X.-F. Ren and G.-P. Guo, Opt. Express, 2016, 24, 27554.

409. T. S. Sreeprasad, P. Nguyen, N. Kim and V. Berry, Nano Lett., 2013, 13, 4434.

410. P. Topolovsek, L. Cmok, C. Gadermaier, M. Borovsak, J. Kovac and A. Mrzel, Nanoscale, 2016, 8, 10016.

411. D. Voiry, J. Yang and M. Chhowalla, Adv. Mater., 2016, 28, 6197.

412. R. Deng, H. Yi, F. Fan, L. Fu, Y. Zeng, Y. Wang, Y. Li, Y. Liu, S. Ji and Y. Su, RSC $A d v ., 2016,6,77083$.

413. R. Bhandavat, L. David and G. Singh, J. Phys. Chem. Lett., 2012, 3, 1523.

414. Y. D. Zhao, J. S. Qiao, P. Yu, Z. X. Hu, Z. Y. Lin, S. P. Lau, Z. Liu, W. Ji and Y. Chai, Adv. Mater., 2016, 28, 2399.

415. M. G. Blamire and J. W. A. Robinson, J. Phys.: Condens. Matter, 2014, 26, 453201.

416. D. A. Dikin, M. Mehta, C. W. Bark, C. M. Folkman, C. B. Eom and V. Chandrasekhar, Phys. Rev. Lett., 2011, 107, 056802.

417. Y. Zhou and E. J. Reed, J. Phys. Chem. C, 2015, 119, 21674.

418. C. Wan, X. Gu, F. Dang, T. Itoh, Y. Wang, H. Sasaki, M. Kondo, K. Koga, K. Yabuki, G. J. Snyder, R. Yang and K. Koumoto, Nat. Mater., 2015, 14, 622.

419. A. K. Geim and I. V. Grigorieva, Nature, 2013, 499, 419.

420. K. S. Novoselov, A. Mishchenko, A. Carvalho and A. H. Castro Neto, Science, 2016, 353, aac9439.

421. E. J. G. Santos, D. Scullion, X. S. Chu, D. O. Li, N. P. Guisinger and Q. H. Wang, Nanoscale, 2017, 9, 13245.

422. H. Huang, Y. Huang, S. Wang, M. Zhu, H. Xie, L. Zhang, X. Zheng, Q. Xie, D. Niu and Y. Gao, Crystals, 2016, 6, 113.

423. J. M. Cai, P. Ruffieux, R. Jaafar, M. Bieri, T. Braun, S. Blankenburg, M. Muoth, A. P. Seitsonen, M. Saleh, X. L. Feng, K. Mullen and R. Fasel, Nature, 2010, 466, 470.

424. J. R. Sanchez-Valencia, T. Dienel, O. Groning, I. Shorubalko, A. Mueller, M. Jansen, K. Amsharov, P. Ruffieux and R. Fasel, Nature, 2014, 512, 61.

425. P. Ruffieux, S. Y. Wang, B. Yang, C. Sanchez-Sanchez, J. Liu, T. Dienel, L. Talirz, P. Shinde, C. A. Pignedoli, D. Passerone, T. Dumslaff, X. L. Feng, K. Mullen and R. Fasel, Nature, 2016, 531, 489.

426. C. H. Lee, T. Schiros, E. J. G. Santos, B. Kim, K. G. Yager, S. J. Kang, S. Lee, J. Yu, K. Watanabe, T. Taniguchi, J. Hone, E. Kaxiras, C. Nuckolls and P. Kim, Adv. Mater., 2014, 26, 2812.

427. Y.-J. Yu, G.-H. Lee, J. I. Choi, Y. S. Shim, C.-H. Lee, S. J. Kang, S. Lee, K. T. Rim, G. W. Flynn, J. Hone, Y.-H. Kim, P. Kim, C. Nuckolls and S. Ahn, Adv. Mater., 2017, 29, 1603925 .

428. R. Phillipson, C. J. Lockhart de la Rosa, J. Teyssandier, P. Walke, D. Waghray, Y. Fujita, J. Adisoejoso, K. S. Mali, I. Asselberghs, C. Huyghebaert, H. Uji-i, S. De Gendt and S. De Feyter, Nanoscale, 2016, 8, 20017.

429. S. Datta, Y. Q. Cai, I. Yudhistira, Z. B. Zeng, Y. W. Zhang, H. Zhang, S. Adam, J. S. Wu and K. P. Loh, Nat. Commun., 2017, 8, 677.

430. E. Orgiu and P. Samorì, Adv. Mater., 2014, 26, 1827.

431. L. Wang and Q. Li, Chem. Soc. Rev., 2018, 47, 1044.

432. X. Zhang, L. Hou and P. Samorì, Nat. Commun., 2016, 7, 11118.

433. M. E. Itkis, X. Chi, A. W. Cordes and R. C. Haddon, Science, 2002, 296, 1443.

434. C. Sporer, I. Ratera, D. Ruiz-Molina, Y. Zhao, J. Vidal-Gancedo, K. Wurst, P. Jaitner, K. Clays, A. Persoons, C. Rovira and J. Veciana, Angew. Chem. Int. Ed., 2004, 43, 5266.

435. C. Simão, M. Mas-Torrent, N. Crivillers, V. Lloveras, J. M. Artés, P. Gorostiza, J. Veciana and C. Rovira, Nat. Chem., 2011, 3, 359.

436. P. Samorì, I. A. Kinloch, X. Feng and V. Palermo, 2D Mater., 2015, 2, 030205. 


\section{Figures}

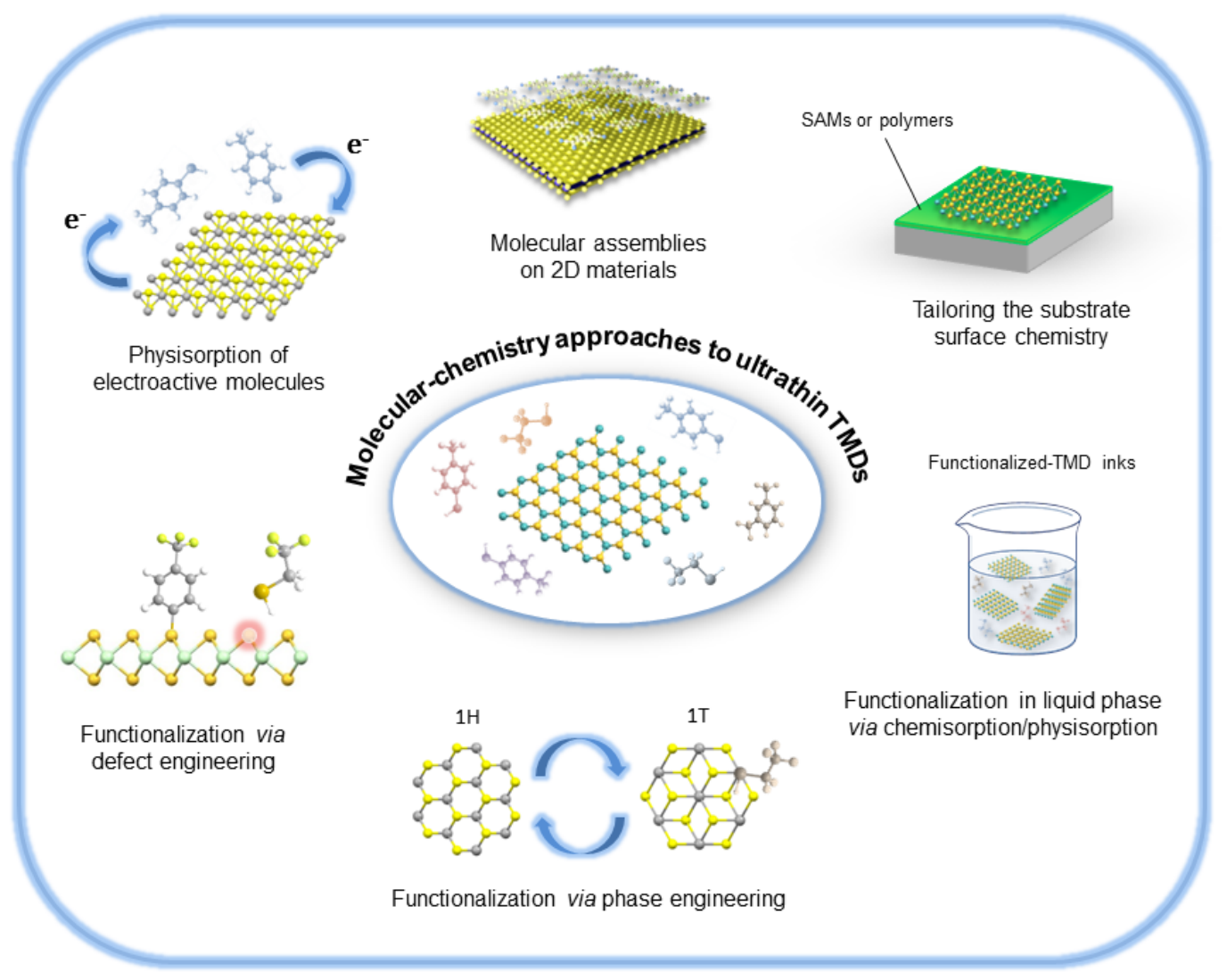

Fig. 1 Schematic representation of the different molecular chemistry approaches that have been investigated in the last years for tailoring the properties of ultrathin TMDs. 
(a) $1 \mathrm{H}$

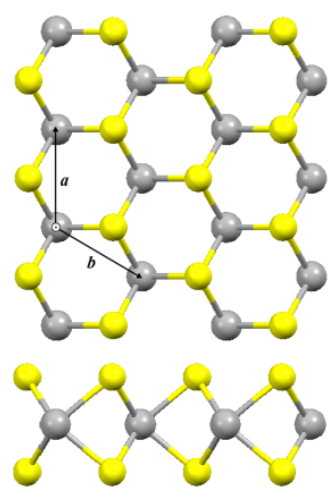

(b)

Trigonal prismatic
$1 \mathrm{~T}$

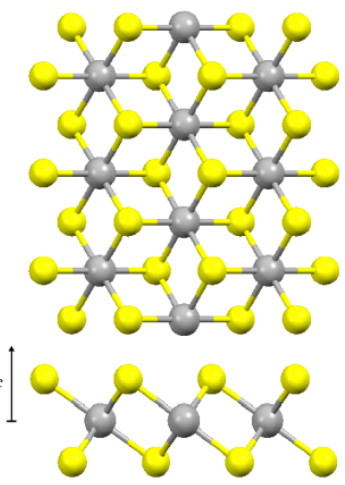

Octahedral (c)

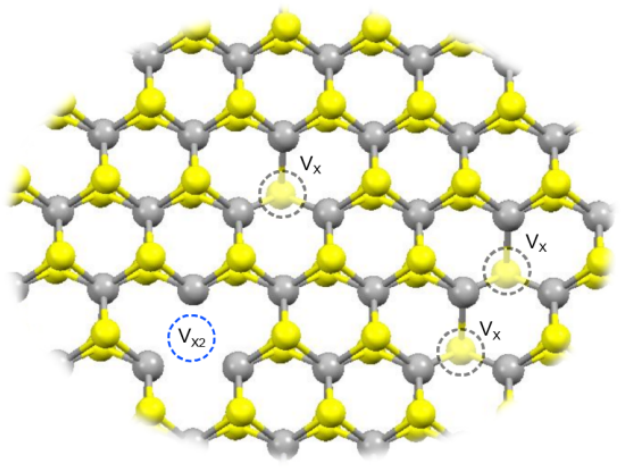

(d)

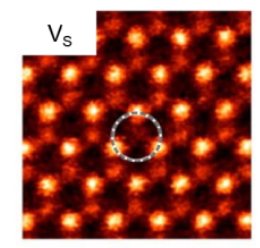

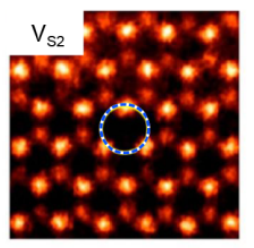

Fig. 2 Crystal structures and defects in monolayer TMDs. (a) Schematic illustration of the $1 \mathrm{H}$ (left) and $1 \mathrm{~T}$ (right) polytypes, as seen along the $c$-axis (top) and $a$-axis (bottom) of the crystal. (b) Three-dimensional representation of the most common coordination geometries between $\mathrm{M}$ and $\mathrm{X}$ atoms. (c) Structure of single $\left(V_{\mathrm{X}}\right)$ and double $\left(V_{\mathrm{X} 2}\right)$ chalcogen vacancies in $1 \mathrm{H}$ TMDs. (d) Atomic-resolution annular dark field (ADF) TEM images of single (left) and double (right) SVs present in monolayer $\mathrm{MoS}_{2}$ sheets grown by CVD. (d) Adapted with permission from ref. 158. Copyright 2013 American Chemical Society. 


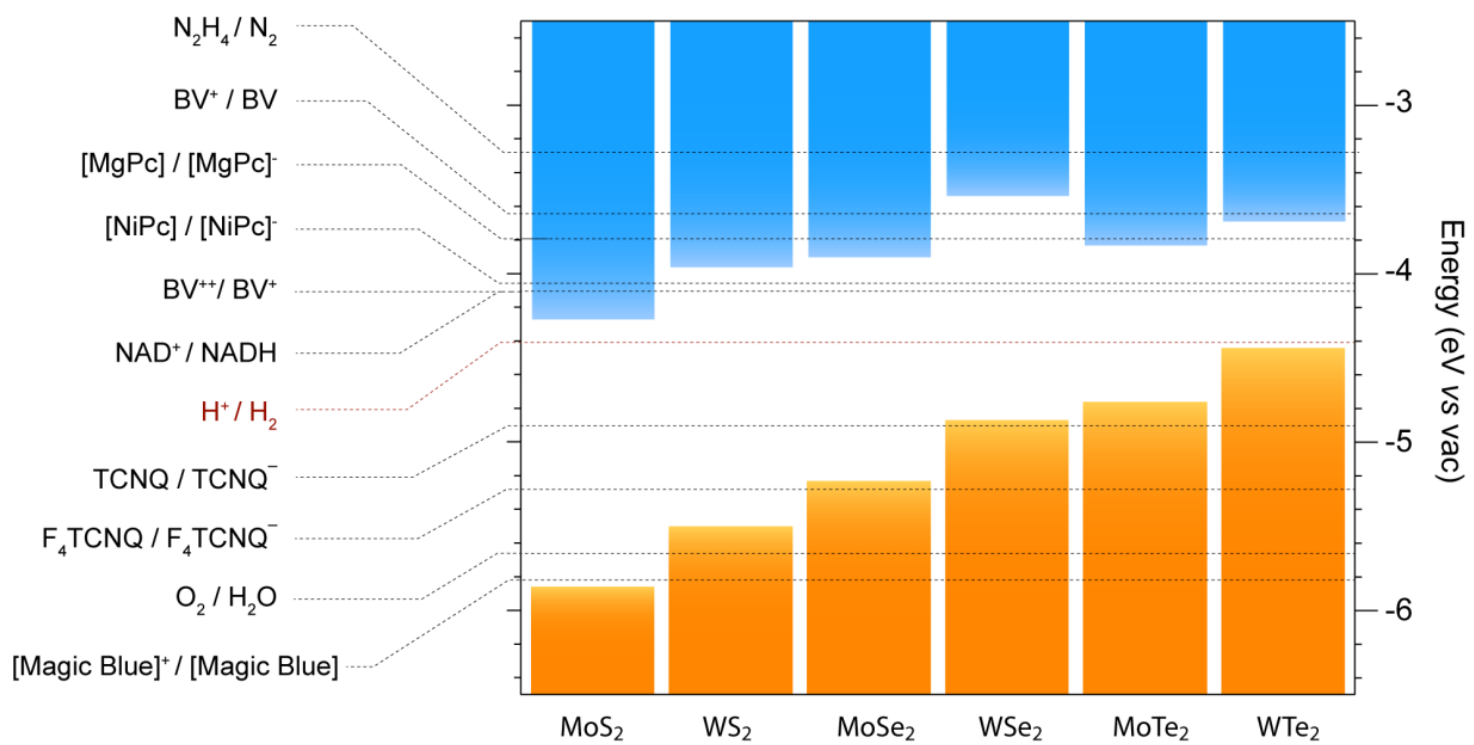

Fig. 3 Comparison between the electrochemical redox potentials of various molecular dopants and the band edges of group-6 TMDs. Approximate reduction potentials (eV vs vacuum) were derived from values reported in literature, namely hydrazine $\left(\mathrm{N}_{2} \mathrm{H}_{4}\right),{ }^{214}$ benzyl viologen $(\mathrm{BV}),{ }^{85}$ magnesium phtalocyanine $(\mathrm{MgPc}),{ }^{99}$ nickel phtalocyanine $(\mathrm{NiPc}),{ }^{99}$ nicotinamide adenine dinucleotide $(\mathrm{NADH}),{ }^{209}$ tetracyanoquinodimethane (TCNQ), ${ }^{209}$ tetrafluoro-7,7,8,8-tetracyanoquinodimethane $\left(\mathrm{F}_{4}\right.$-TCNQ),${ }^{209}$ tris(4bromophenyl)-ammoniumyl hexachloroantimonate (Magic Blue). ${ }^{201}$ The CBM/VBM band edges (theoretical values) were taken from ref. 210. 
(a)

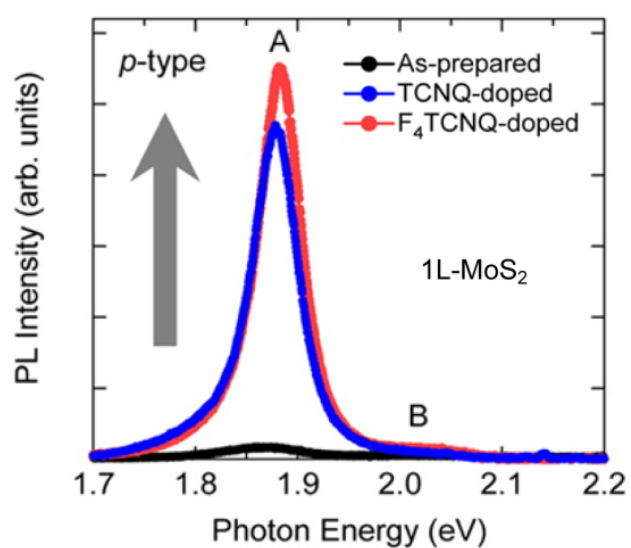

(c)

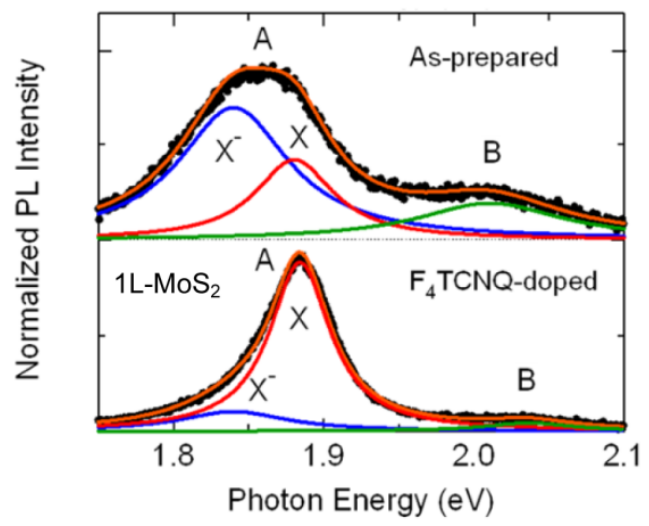

(b)

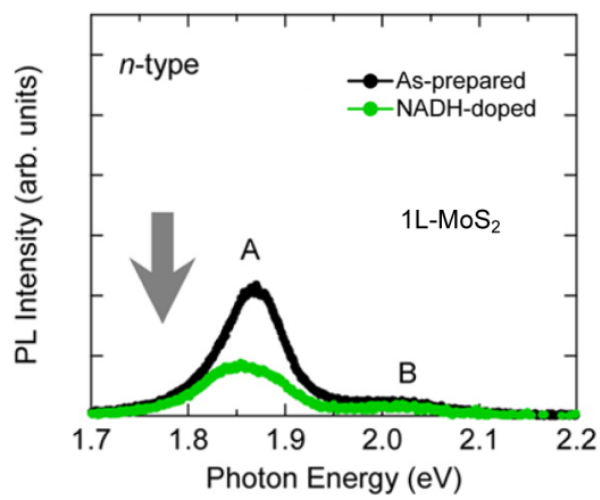

(d)

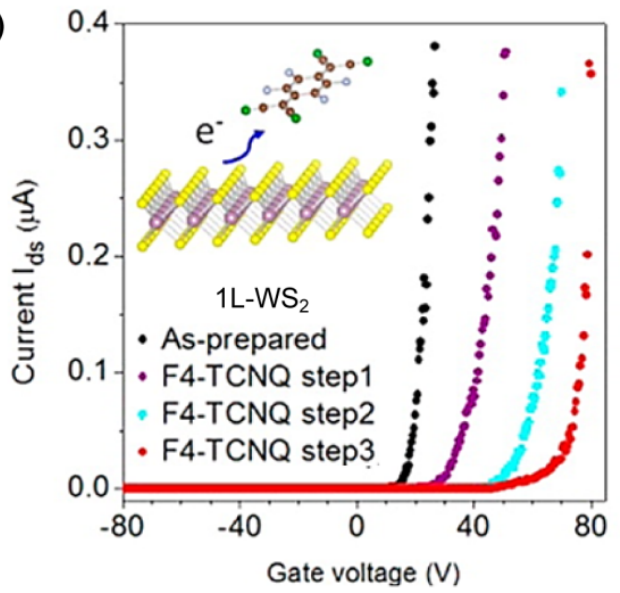

Fig. 4 Modulation of PL and electrical transport via molecular physisorption. (a) Effect of $p$-dopant TCNQ and $\mathrm{F}_{4}$-TCNQ molecules, as well as (b) $n$-dopant NADH molecules on the PL spectra of monolayer (1L) $\mathrm{MoS}_{2}$ sheets prepared by micromechanical cleavage. (c) Fitting of the PL spectra of as-prepared and $\mathrm{F}_{4}$-TCNQ-doped 1L-MoS 2 . The A peaks were fitted with trion $\left(\mathrm{X}^{-}\right)$and neutral exciton $(\mathrm{X})$ contributions. (d) Transfer curves of a $1 \mathrm{~L}-\mathrm{WS}_{2}$ FET for different concentrations of $\mathrm{F}_{4}$-TCNQ molecules. (a-c) Adapted with permission from ref. 209. Copyright 2013 American Chemical Society. (d) Adapted with permission from ref. 216. Copyright 2014 American Chemical Society. 


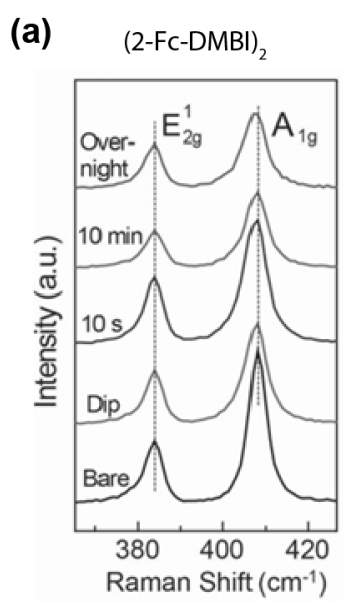

(c)

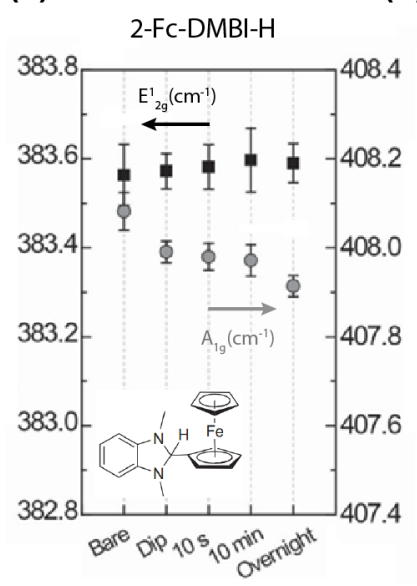

(b)

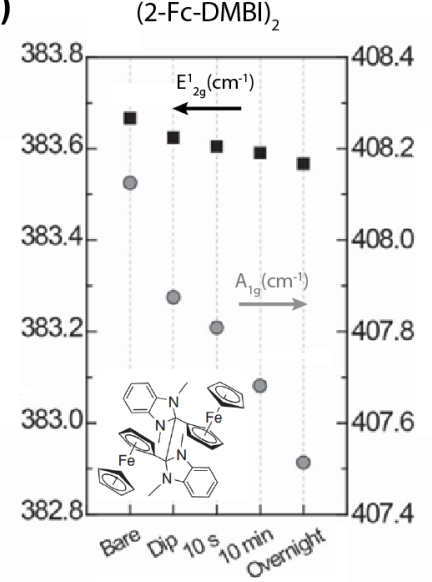

(d)

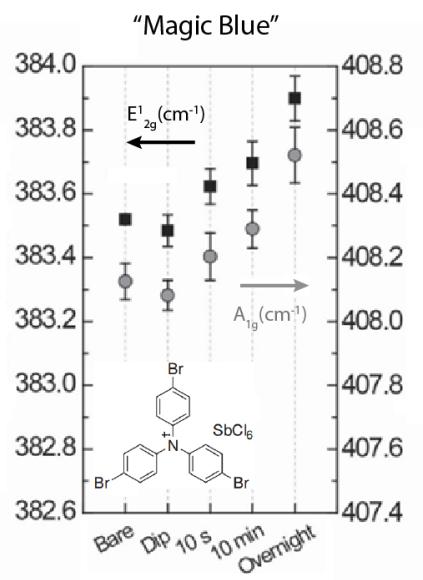

Fig. 5 Influence of physisorbed molecules on the Raman spectra of TMDs. (a) Raman spectra of few-layer $\mathrm{MoS}_{2}$ sheets before and after different treatment with (2-Fc$\mathrm{DMBI})_{2}$. (b-d) $E_{2 \mathrm{~g}}^{1}$ (squares) and $A_{1 \mathrm{~g}}$ (circles) peak position shifts upon doping with (2Fc-DMBI) 2 , 2-Fc-DMBI-H and "Magic Blue", respectively. (a-d) Adapted with permission from ref. 87. Copyright 2015 Wiley-VCH. 
(a)

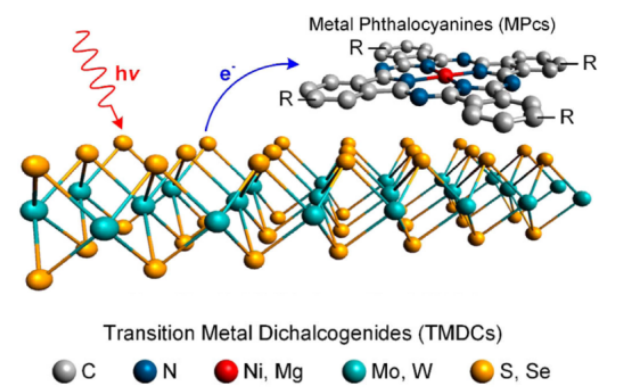

(b)

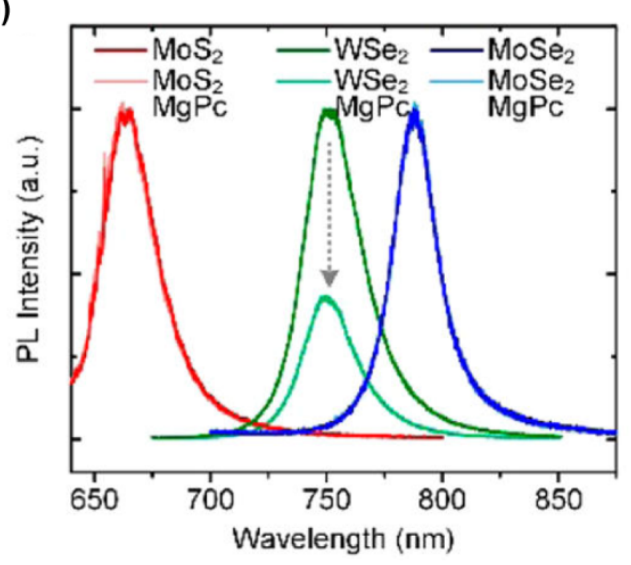

(c)

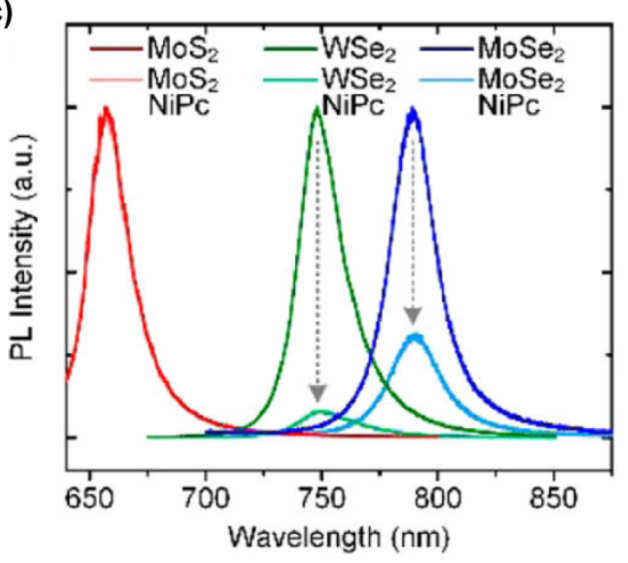

Fig. 6 Doping of TMDs by MPcs. (a) Schematic representation of a TMD sheet and a metallophthalocyanine molecule (MPc). (b, c) PL spectra of $\mathrm{MoS}_{2}, \mathrm{MoSe}_{2}$, and $\mathrm{WSe}_{2}$ before and after physisorption of (b) NiPc and (c) MgPc. (a-d) Reprinted with permission from ref. 99. Copyright 2016 American Chemical Society. 
(a)

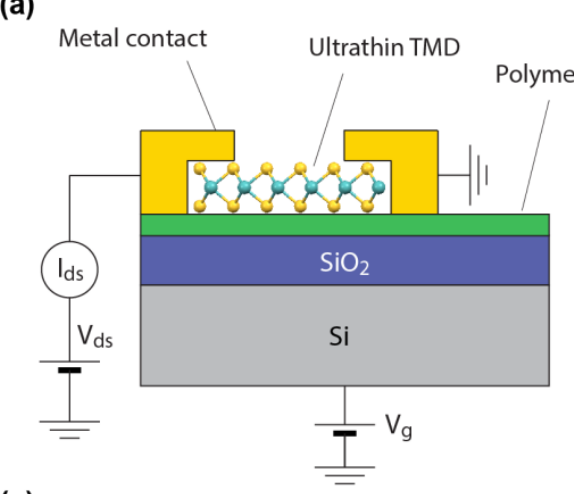

(c)

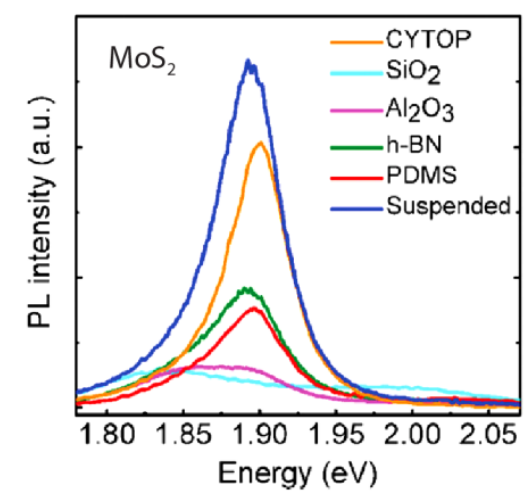

(b)

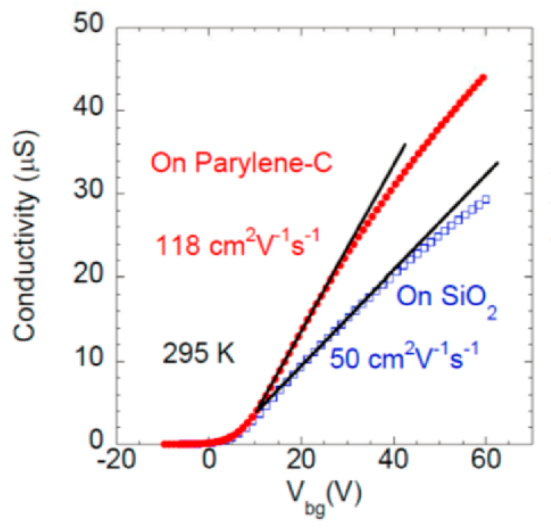

(d)

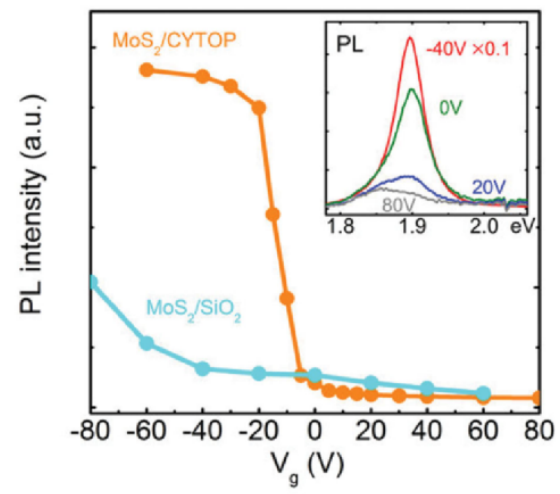

Fig. 7 Effects of polymer substrates on TMDs. (a) Structure of the FET device used to investigate the effects of polymer films on the charge-transport properties of ultrathin TMDs. (b) Transfer characteristics of few-layers $\mathrm{MoSe}_{2}$ FETs built on parylene and $\mathrm{SiO}_{2}$ substrates. (c) PL spectra of monolayer $\mathrm{MoS}_{2}$ sheets deposited on different substrates. (d) Gate-voltage dependence of the PL intensity of monolayer $\mathrm{MoS}_{2}$ on Cytop and $\mathrm{SiO}_{2}$. Inset: PL spectra of monolayer $\mathrm{MoS}_{2}$ on Cytop at various gate voltages. (b) Adapted with permission from ref. 205. Copyright 2014 American Chemical Society. (c, d) Adapted with permission from ref. 206. Copyright 2016 Wiley-VCH. 
(a)

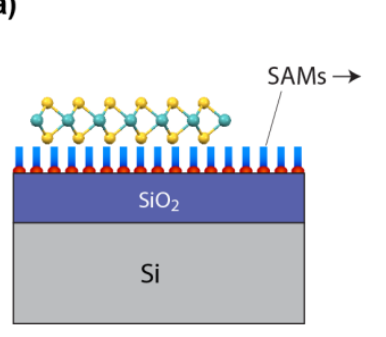

OTS

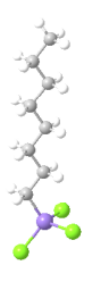

FOTS
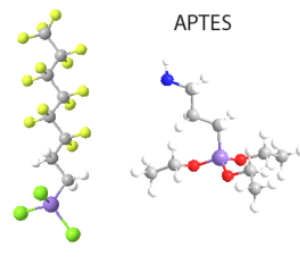

Si (c)

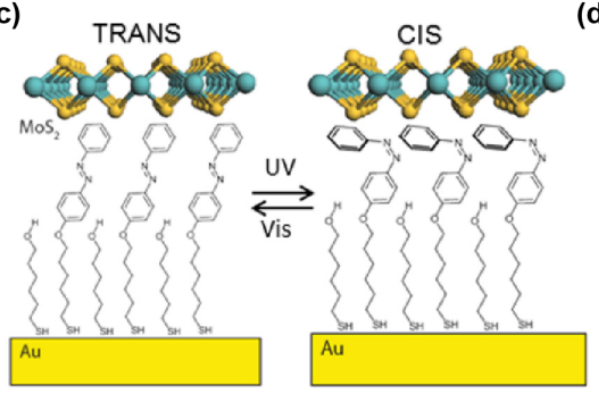

(d)

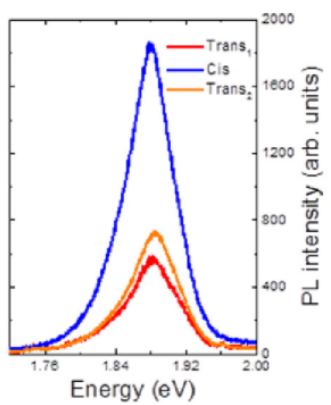

(b)

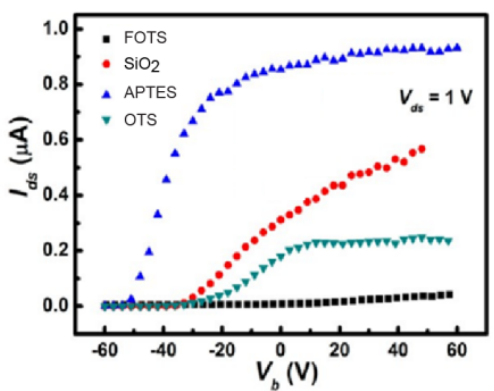

(e)

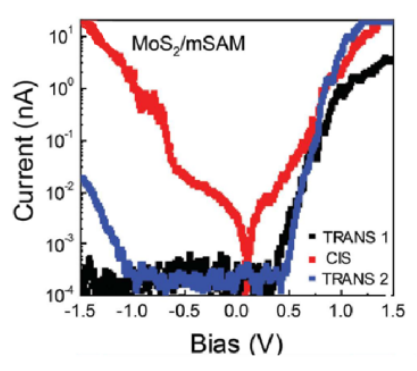

Fig. 8 The effect of SAM-modified substrates on TMDs. (a) Highly ordered molecules within the SAM are chemisorbed on the substrate surface and interact through weak van der Waals forces with TMD sheets deposited onto them. The chemical structure of the three silane coupling agents used in ref. 84 is also shown. (b) Transfer characteristics of few-layers $\mathrm{MoS}_{2}$ onto the different SAMs. (c) Cartoon of photoswitchable AZO-SAM on gold substrates interacting with $\mathrm{MoS}_{2}$. (d) Modification of the $\mathrm{MoS}_{2}$ PL intensity accompanying the AZO-SAM switch. (e) Light-induced modulation of the electrical current flowing vertically across the SAM (from gold to $\mathrm{MoS}_{2}$ ). (b) Adapted with permission from ref. 84. Copyright 2013 American Chemical Society. (c, d) Reprinted with permission from ref. 222. Copyright 2014 American Institute of Physics. (e) Adapted with permission from ref. 247. Copyright 2015 Wiley-VCH. 

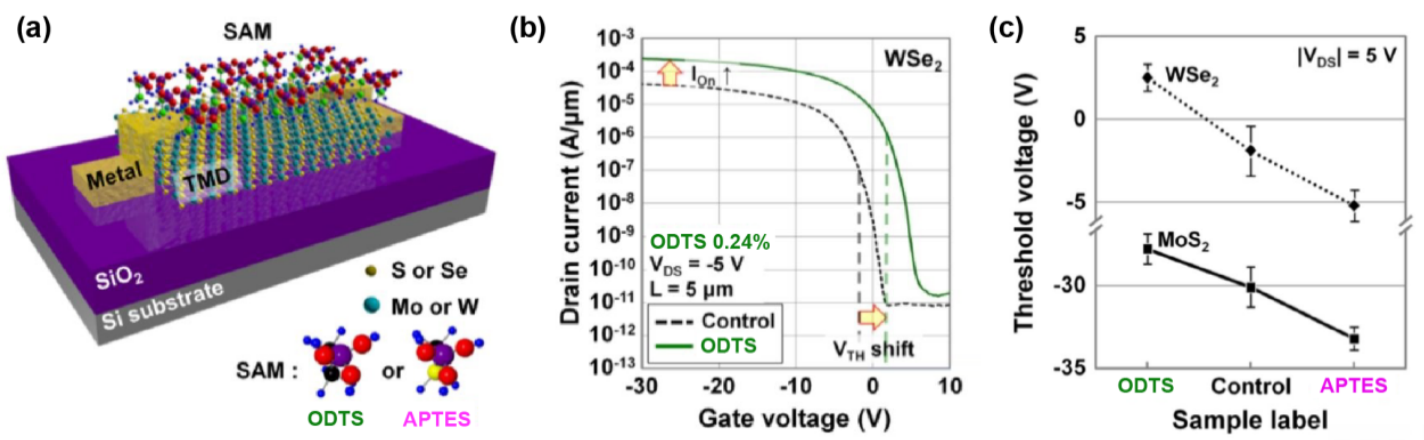

Fig. 9 Highly-ordered molecular layers on TMDs. (a) Schematic diagram of back-gated TMD transistors modified by ODTS or APTES treatments of the top surface. (b) Transfer characteristics of clean and ODTS-treated few-layer WSe $\mathrm{WETs}_{2}$ built on $\mathrm{SiO}_{2}$ substrates. (c) Threshold voltage shifts measured in few-layer TMD FETs treated with different molecules. Adapted with permission from ref. 39. Copyright 2015 Wiley$\mathrm{VCH}$. 
(a)

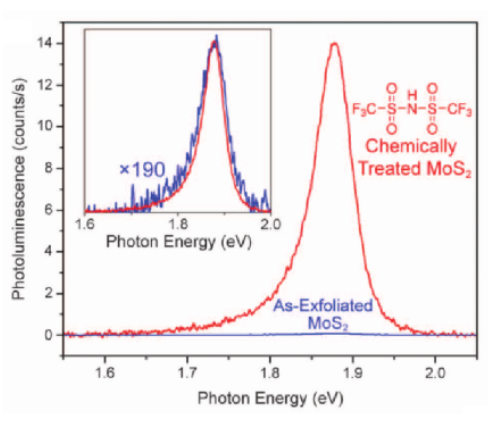

(d)

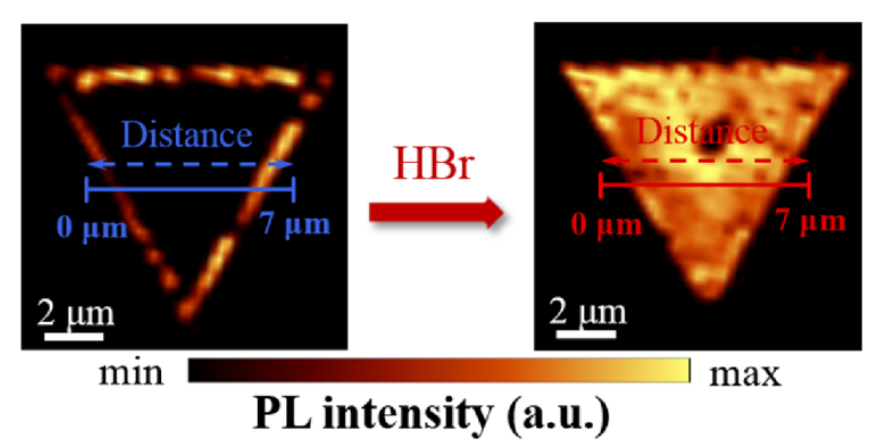

(b)

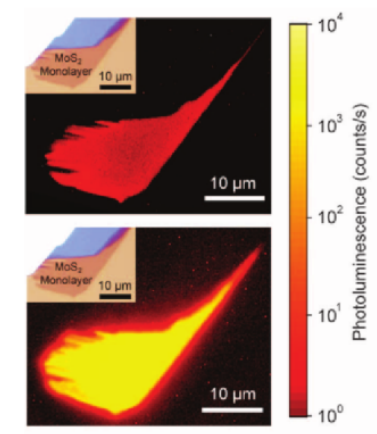

(e)

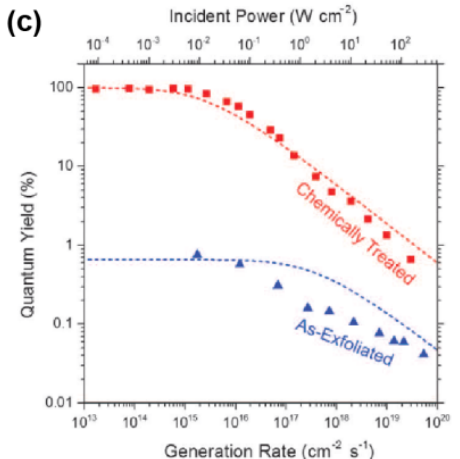

(f)

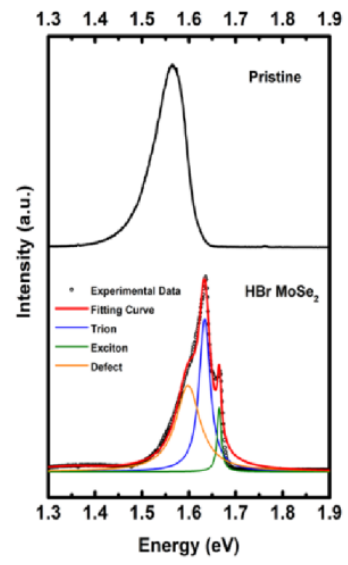

Fig. 10 Enhancement of the TMD PL by acid treatments. (a) PL spectrum for asexfoliated and TFSI-treated $\mathrm{MoS}_{2}$ monolayers. Inset: normalized spectra. The chemical structure of TFSI is also shown. (b) PL intensity maps of the same $\mathrm{MoS}_{2}$ monolayer before and after the TFSI treatment. (c) Quantum yield dependence on the pump power, extracted from calibrated photoluminescence measured at different incident power. (d-e) PL intensity maps of an individual CVD-grown $\mathrm{MoSe}_{2}$ monolayer before and after the $\mathrm{HBr}$ treatment. (f) PL spectra of pristine and $\mathrm{HBr}$-treated $\mathrm{MoSe}_{2}$ flake. (a-c) Reprinted with permission from ref. 97. Copyright 2015 AAAS. (d-f) Reprinted with permission from ref. 100. Copyright 2016 American Chemical Society. 
Electron irradiation
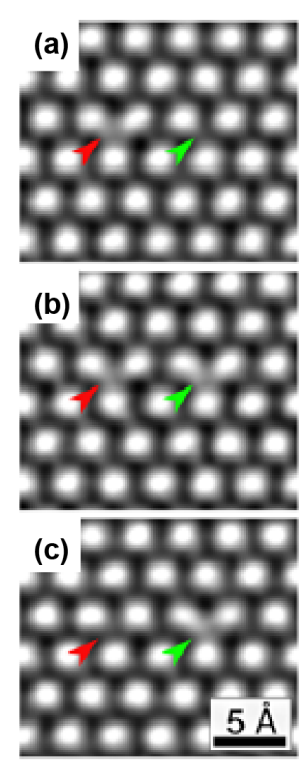

Argon-ion irradiation

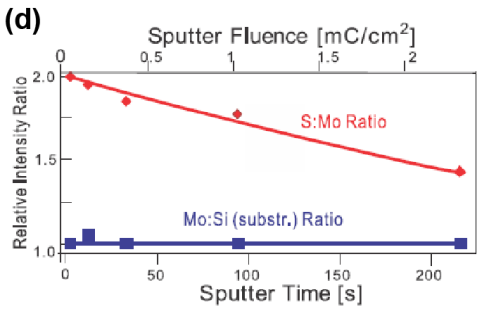

(f)

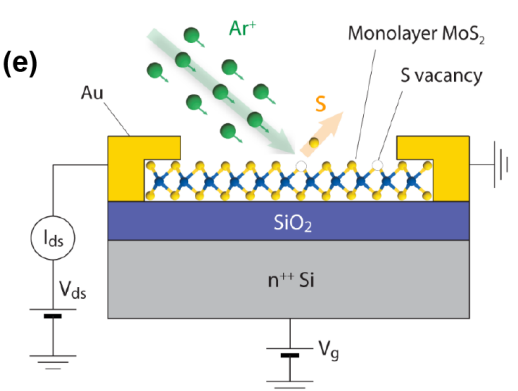

(g)

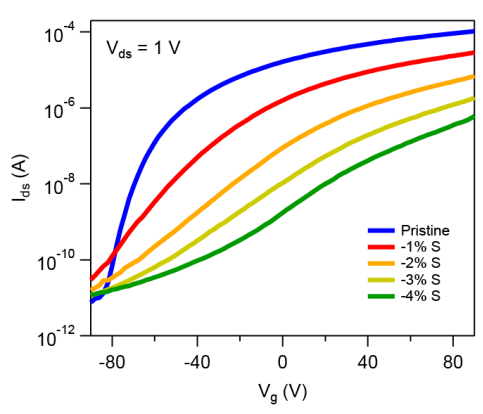

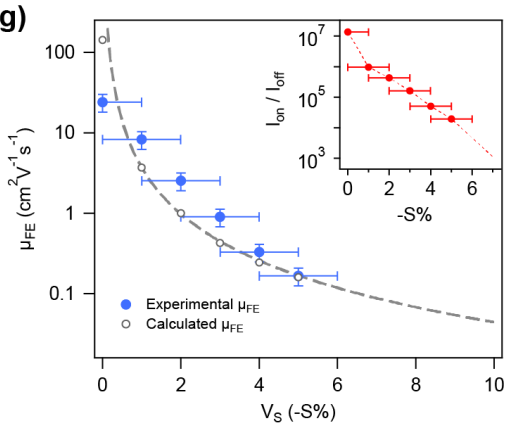

Fig. 11 Engineering chemically-active defects in ultrathin TMDs. (a-c) Aberrationcorrected HR-TEM images demonstrating the generation of SVs via electron irradiation at $80 \mathrm{keV}$ and the subsequent filling by impurity atoms. The red arrow in (a) indicates an initial SV that is filled by an impurity atom between (b) and (c), whereas the green arrow indicates a S atom that is ejected between (a) and (b) leaving a single vacancy. (d) Evolution of the stoichiometric ratio - as obtained from XPS measurements - of largeare $\mathrm{CVD} \mathrm{MoS}_{2}$ films exposed to an increasing dose of low-energy $(\mathrm{E} \approx 500 \mathrm{eV})$ argon ions. (e) Schematics of the ion-bombardment experiment performed on monolayer $\mathrm{MoS}_{2}$ FETs. (f) Evolution of the transfer characteristics of the FET schematized in (e) with increasing density of SVs. (g) Experimental and theoretical evolution of the field-effect mobility $\mu_{\mathrm{FE}}$ and $I_{\mathrm{on}} / I_{\mathrm{off}}$ ratio (inset) with SV density. (a-c) Reprinted with permission from ref. 68. Copyright 2012 American Physical Society. (d) Reproduced with permission from ref. 274. Copyright 2013 IOP Science. (e-g) Reproduced with permission from ref. 109. Copyright 2017 Wiley-VCH. 

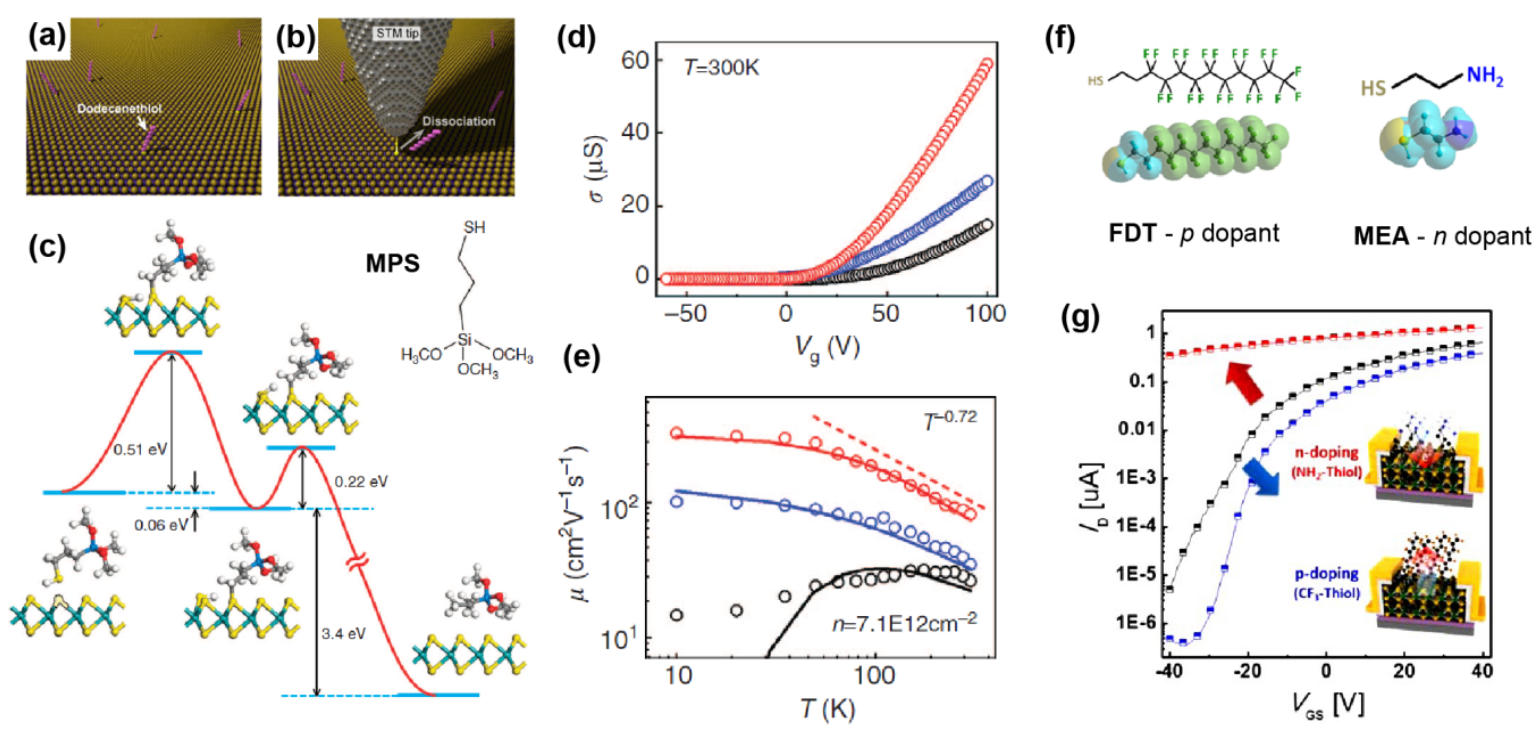

Fig. 12 Repair/functionalization of chemically-active defects in $\operatorname{MoS}_{2}$. (a, b) Schematic representation of the STM experiments on the adsorption (a) and dissociation (b) of thiol molecules on the surface of a bulk $2 \mathrm{H}-\mathrm{MoS}_{2}$ crystal. (c) Kinetics and transient states of the reaction between a single SV and MPS. (d, e) Effect of the MPS treatment on the transfer characteristics (d) and field-effect mobility (e) of monolayer $\mathrm{MoS}_{2}$ FETs. Black: no treatment. Blue: top surface treatment. Red: bottom and top surface treatment. (f) Molecular dopants with thiol anchoring groups. The molecules have same spacer group (two carbon atoms) between the doping and anchoring units. (g) Transfer characteristics of untreated (black), FDT-treated (blue) and MEA-treated (red) few-layer MoS $_{2}$ FETs. (a, b) Adapted with permission from ref. 181. Copyright 2012 American Chemical Society. (c-e) Adapted with permission from ref. 182. Copyright 2014 Nature Publishing Group. (f, g) Adapted with permission from ref. 88. Copyright 2015 American Chemical Society 
(a)
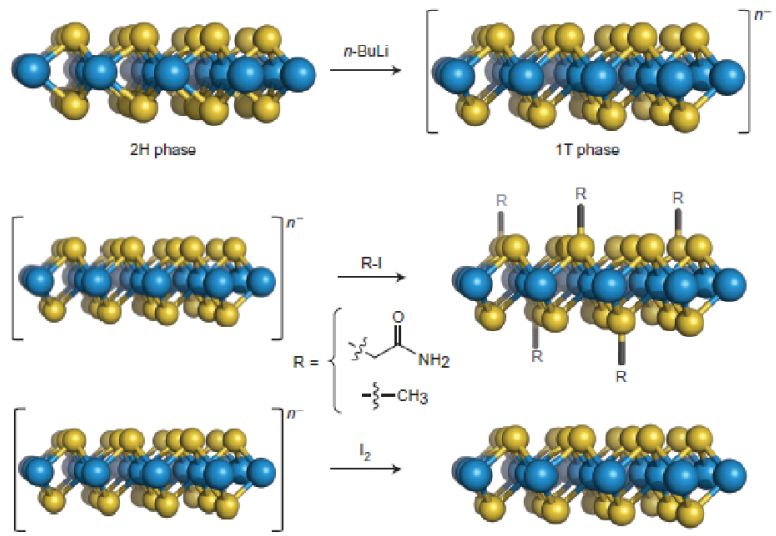

(b)
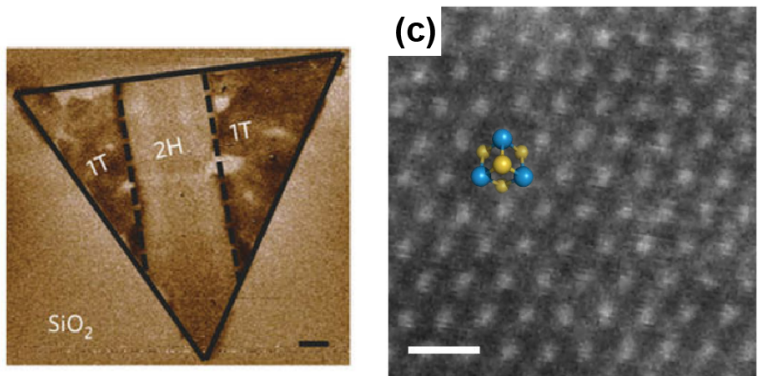

(d)

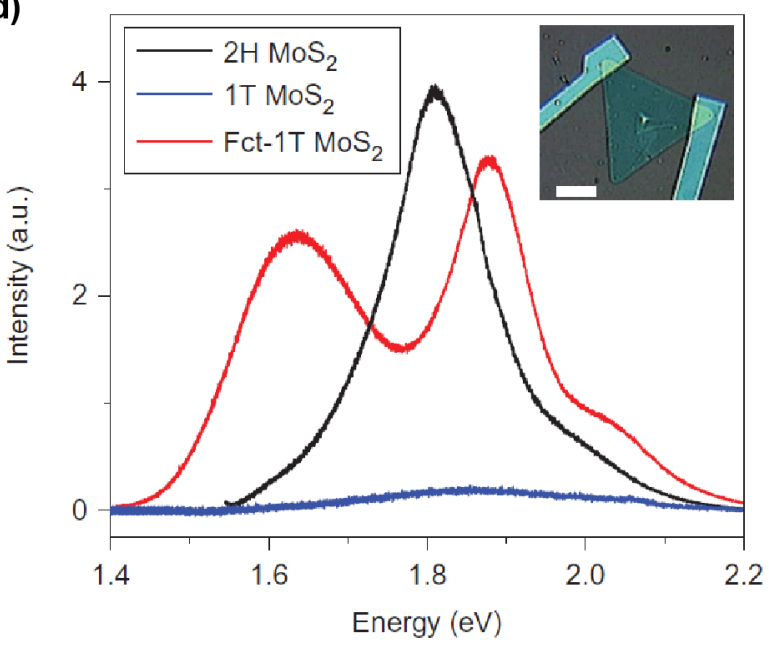

Fig. 13 Covalent functionalization of $\mathrm{MoS}_{2}$ via phase engineering. (a) Schematics of the functionalization approach based on the reaction of $1 \mathrm{~T}-\mathrm{MoS}_{2}$ nanosheets with organohalides. (b) Electrostatic force microscopy phase image of a CVD-grown $\mathrm{MoS}_{2}$ sheet with locally patterned metallic and semiconducting regions. Scale bar: $1 \mu \mathrm{m}$. (c) High annular angle dark field scanning TEM images of functionalized monolayer $\mathrm{MoS}_{2}$ sheets with octahedral coordination. Scale bar: $0.5 \mathrm{~nm}$. (d) PL spectra of a CVD-grown $\mathrm{MoS}_{2}$ sheet at different stages of the functionalization process: pristine (black), after exposure to $n$-butyllithium (blue) and after functionalization (red). (a, c, d) Adapted with permission from ref. 80. Copyright 2015 Nature Publishing Group. (b) Reproduced with permission from ref. 308. Copyright 2014 Nature Publishing Group. 


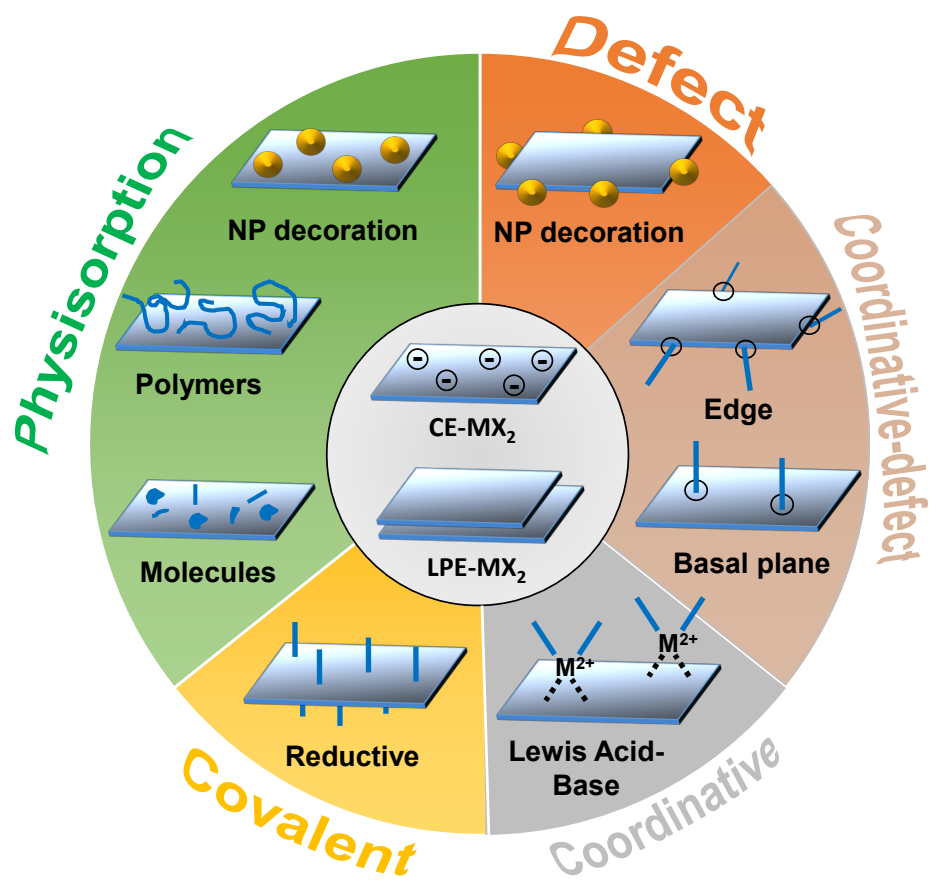

Fig. 14 Overview of functionalization strategies for TMDs in the liquid phase based on chemically exfoliated $\left(\mathrm{CE}-\mathrm{MX}_{2}\right)$ and liquid phase exfoliated (LPE-MX $\left.\mathrm{M}_{2}\right)$ nanosheets. In addition to physisorption, different approaches for chemisorption exist. These can be classified as covalent (involving $\mathrm{X}-\mathrm{C}$ bonds), coordinative (involving chalcogens as ligands) and defect functionalization. Defects such as chalcogen vacancies can also be used for coordination chemistry involving dative bonds with nucleophiles. 
(a) Functionalization of chemically exfoliated $\mathrm{MX}_{2}$

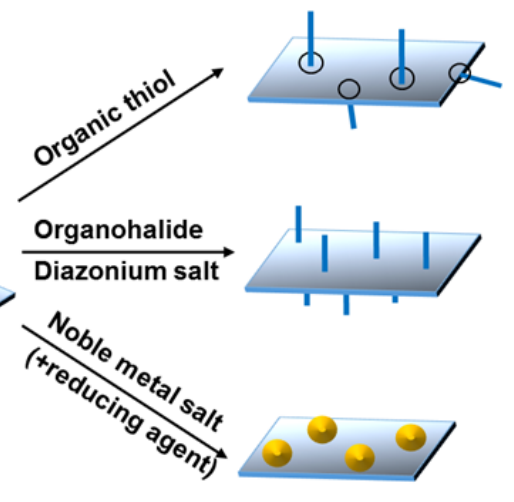

(b) Functionalization of liquid exfoliated $\mathrm{MX}_{2}$
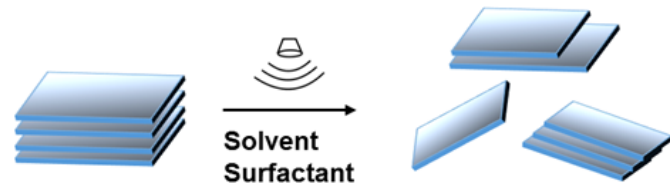

2H-LPE-MX
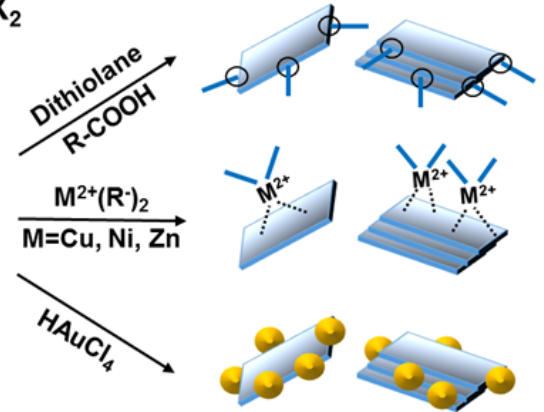

Fig. 15 Schematic of reaction pathways to achieve functionalization via chemisorption. (a) Pathways building on chemical exfoliation via intercalation with n-butyllithium. This process yields negatively charged $\mathrm{MX}_{2}$ nanosheets in the $1 \mathrm{~T} / 1 \mathrm{~T}^{\prime}$ polytype which can be further derivatized via i) coordination of thiols to chalcogen vacancy sites (defect functionalization), ii) reductive covalent functionalization with electrophiles such as organohalides or diazonium salts, or iii) physisorption of metal nanoparticles. (b) Pathways building on liquid phase exfoliation in appropriate solvents or aqueous surfactants. This strategy yields few-layered nanosheets of the semiconducting $2 \mathrm{H}-$ polytype. These can be functionalized by i) coordination of electron donors (dithiolanes or carboxylic acids) to sulfur deficient edges, ii) coordination to metal complexes using the chalcogen as ligand, or iii) nanoparticles covalently bound to nanosheet edges. 

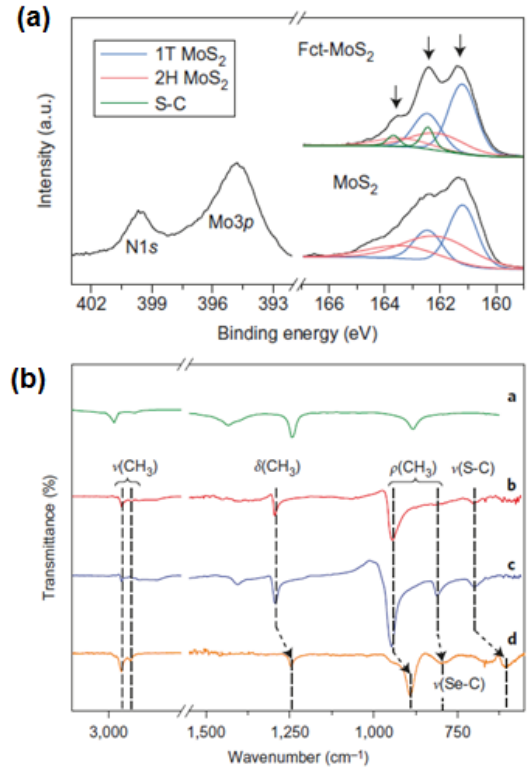

(c)

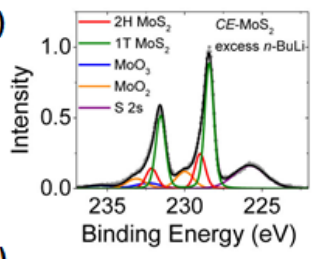

(d)

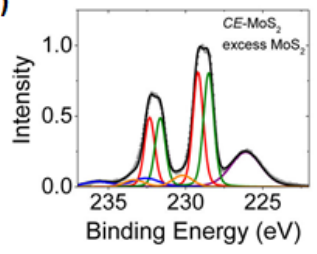

(e)

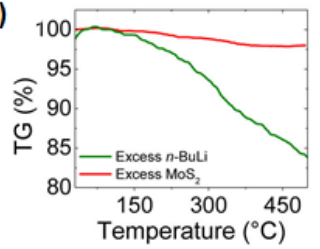

(f)

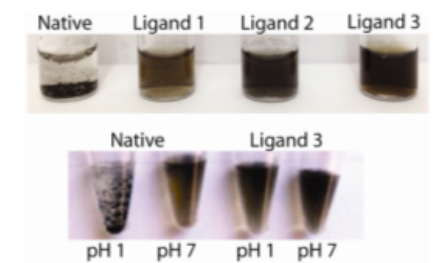

(g)

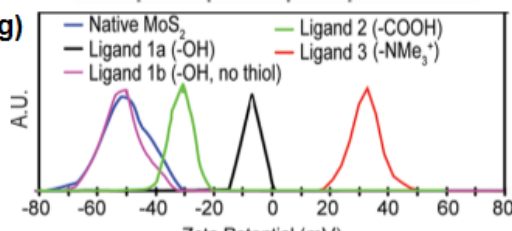

Zeta Potential $(\mathrm{mV})$

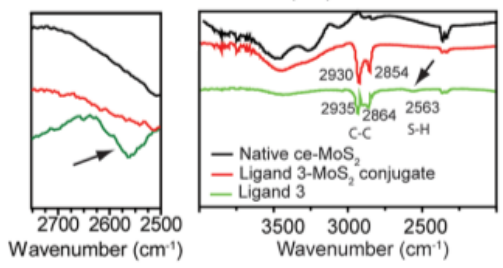

Fig. 16 Covalent reductive and coordinative functionalization of $\mathrm{MX}_{2}$ in liquids. (a, b) Reductive covalent functionalization according to ref. 80. (a) Sulfur XPS core level spectra showing evidence for an additional sulfur species due to the formation of a covalent S-C bond. (b) IR spectra $1 \mathrm{~T}-\mathrm{MoWSe}_{2}$ reductively functionalized with iodomethane. From top to bottom: iodomethane (green), functionalized 1T-MoS 2 (red), $1 \mathrm{~T}-\mathrm{WS}_{2}$ (blue) and $1 \mathrm{~T}-\mathrm{MoSe}_{2}$ (orange). (c-e) Tuning of $2 \mathrm{H} / 1 \mathrm{~T}$ content in $\mathrm{MoS}_{2}$ via intercalation conditions. (c, d) Mo core level spectra after n-BuLi intercalation using (c) excess $\mathrm{n}$-BuLi over $\mathrm{MoS}_{2}$ and (d) excess $\mathrm{MoS}_{2}$ over n-BuLi. (e) Thermogravimetric weight loss of the samples in (c, d) showing negligible weight loss in the case of the intercalation with excess MoS2 suggesting lower defect contents. (f, g) Coordination of thiols to vacancy sites from ref. 259. (f) Top: photographs of $1 \mathrm{~T}-\mathrm{MoS}_{2}$ dispersions after reaction with various ligands, bottom: corresponding zeta potential changes from negative to positive potentials depending on the ligand. (g) IR spectra of the samples showing the disappearance of the S-H vibration (left), but the presence of the rest of the ligand (right). (a,b) Reproduced with permission from ref. 80. Copyright 2015 Nature Publishing Group. (c-e) Reproduced with permission from ref. 260. Copyright 2015 American Chemical Society. (f, g) Reproduced with permission from ref. 259. Copyright 2013 American Chemical Society. 
(a)

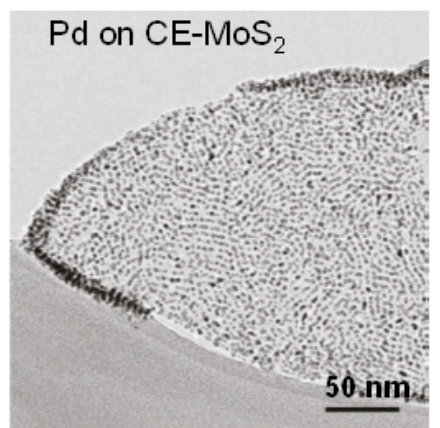

(b)

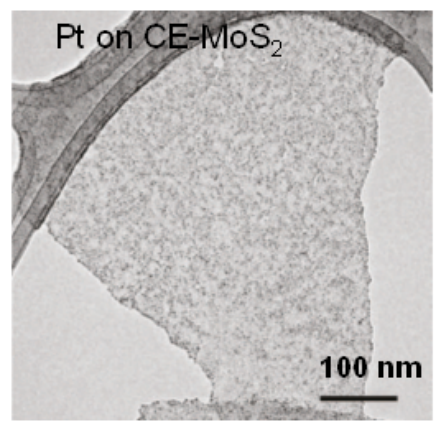

(c)

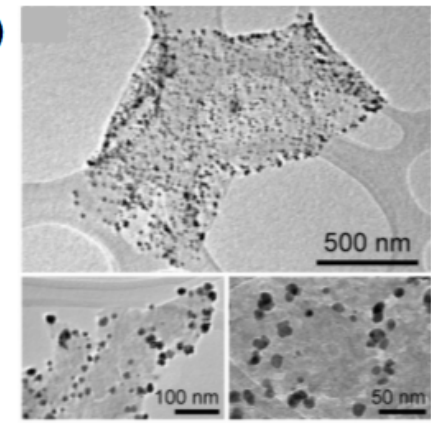

(d)

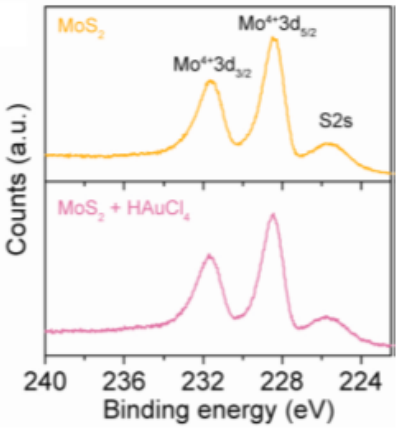

(e)

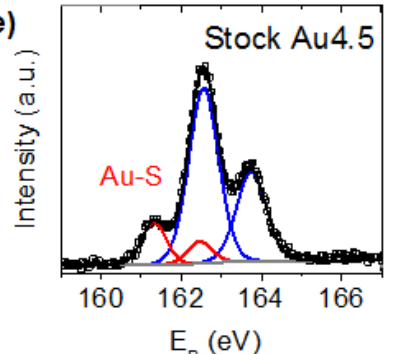

(f)

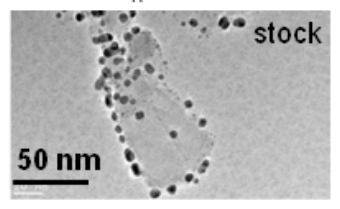

(g)

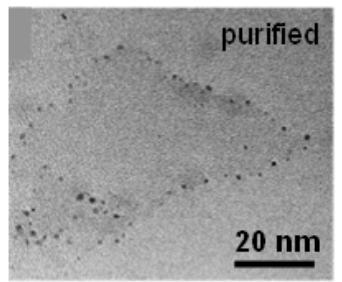

Fig. $17 \mathrm{MX}_{2}$ nanoparticle decoration. (a-d) Nanoparticles epitaxially grown on 1T$\mathrm{MoS}_{2}$. (a-c) TEM images of (a) Pd on $1 \mathrm{~T}-\mathrm{MoS}_{2}$, (b) Pt on $1 \mathrm{~T}-\mathrm{MoS}_{2}$, (c) Au on 1T$M_{o}$. (d) XPS Mo core level spectra showing no changes to the $\mathrm{MoS}_{2}$ on reaction with the metal precursor. (e-g) Au nanoparticles grown on LPE-2H-WS $\mathrm{W}_{2}$ from ref. 404. (e) XPS sulfur core level spectra showing evidence for covalent binding of the nanoparticles to the $\mathrm{WS}_{2}$. (f, g) TEM images of Au-decorated LPE-2H-WS 2 before (f) and after (g) purification. (a, b) Reproduced with permission from ref. 394. Copyright 2013 Nature Publishing Group. (c, d) Reproduced with permission from ref. 395. Copyright 2013 American Chemical Society. (e-g) Reproduced with permission from ref. 404. Copyright 2018 Nature Publishing Group. 
(a)
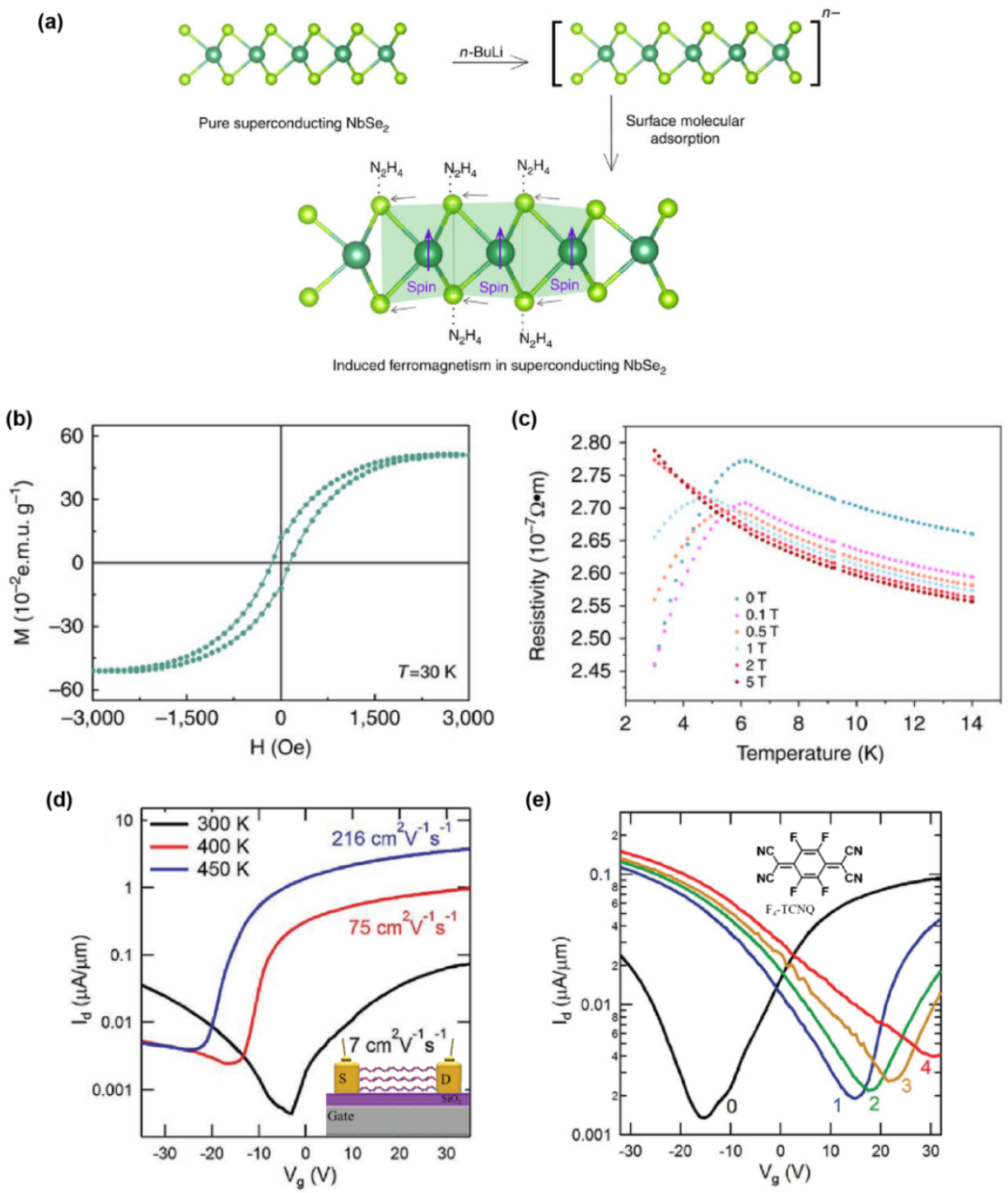

Fig. 18 Tailoring TMDs "beyond MoWSeS" via molecular physisorption. (a) $2 \mathrm{H} \mathrm{NbSe}_{2}$ nanosheets are exfoliated via lithium-ion intercalation. Physisorbed hydrazine molecules trigger a structural distortion of the crystal together with novel ferromagnetic properties in superconducting $\mathrm{NbSe}_{2}$. (b) Magnetization vs magnetic field $(\mathrm{M}-\mathrm{H})$ curves of hydrazine-treated $\mathrm{NbSe}_{2}$ at $30 \mathrm{~K}$. (c) Temperature-dependent electrical resistivity $(R-T$ curves) of hydrazine-treated $\mathrm{NbSe}_{2}$ under different magnetic fields. (d) Transfer curves of a $\mathrm{PdSe}_{2}$ FET showing a progressive conversion from ambipolar to unipolar $n$-type transport upon thermal annealing. (e) Transfer curves of a PdSe 2 FET showing a continuous change from ambipolar to unipolar $p$-type transport via $\mathrm{F}_{4}$-TCNQ physisorption doping. (a-c) Reprinted with permission from ref. 114. Copyright 2016 Nature Publishing Group. (d-e) Reprinted with permission from ref. 113. Copyright 2017 Wiley-VCH. 

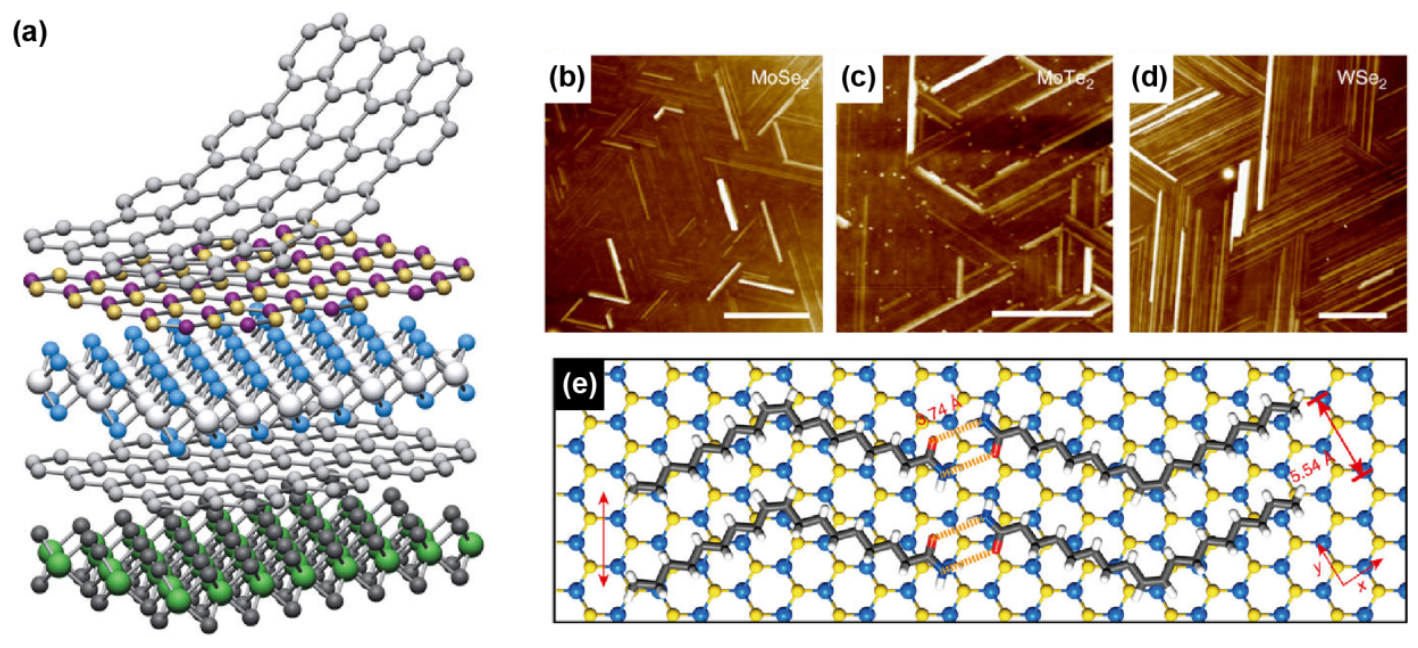

Fig. 19 Hybrid van der Waals heterostructures. (a) TMDs and other 2D Materials can be superimposed to construct a variety of layered structures with opto electronic properties fundamentally different from those of the isolated sheets. (b-d) AFM images of a highly-ordered molecular monolayers on the surface of $\mathrm{MoSe}_{2}$ (b), $\mathrm{MoTe}_{2}$ (c), $\mathrm{WSe}_{2}$ (d). (e) Schematics of the nanoscale molecular arrangement giving rise to the lamellar assembly observed in panels (b-d). Analogous molecular monolayers could be integrated within inorganic van der Waals heterostructures (a) to obtain hybrid organic/inorganic materials with unique properties. (a) Reprinted with permission from ref. 419. Copyright 2013 Nature Publishing Group. (b-e) Reprinted with permission from ref. 252. Copyright 2016 American Institute of Physics. 